LA-8536-MS

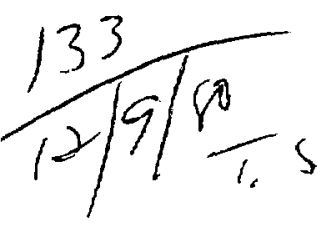

Dr.2107

\title{
The Theory of Contributon Transport
}

正

世

$\frac{2}{\frac{2}{9}}$

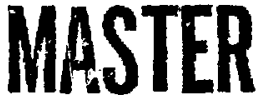




\title{
The Theory of Contributon Transport
}

\author{
J. W. Painter \\ S. A. W. Gerstl \\ G. C. Pomraning*
}

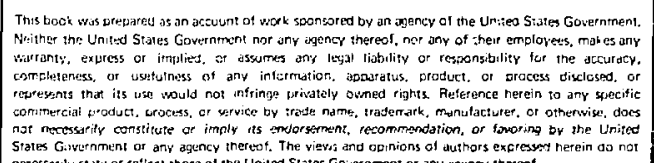

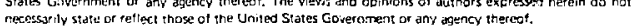

"Consultant. Department of Nuclear Engineering, University of California, Los Angeles, CA 90024.

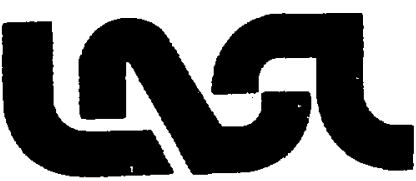


THE THEORY OF CONTRIBUTON TRANSPORT

by

J. W. Painter, S. A. W. Gerstl and

G. C. Pomraning

\section{ABSTRACT}

A general discussion of the physics of contributon transport is presented. To facilitate this discussion, a Boltzmann-like transport equation for contributons is obtained, and special contributon cross sections are defined. However, the main goal of this study is to Identify contributon transport equations and Investigate possible deterministic solution techniques. Four approaches to the deterministic solution of the contributon transport problem are investigated. These approaches are an attempt to exploit certain attractive properties of the contributon flux, $\psi=\phi \phi^{+}$, where $\phi$ and $\phi^{+}$are the solutions to the forward and adjoint Boitzmann transport equations. The first approach involves the derivation of a quasi-1inear differential equation for $\psi$. The order of the resulting differential equation is shown to depend upon $S$ and $\mathrm{S}^{+}$, the source terms, including scattering, in the forward and adjolnt Boltzmann equations. Some simple pure absorber problems are solved analytically. Attemps at numerical solutions for a more general class of problems indicate that when spatial discontinulties exist in $S$ or $S^{+}$, the difference equations are susceptible to numerical Instability with respect to the size or the spatial mesh intervals. The second approach involves the derivation of an explicttly nonlinear differential equation for $\psi$. This equation is a generalization to scattering media of a simflar equation, which was previously obtained for a pure absorber. This approach has not been actively pursued in detail. The third approach involves iterating between a differential equation that contains only $\psi$ and $\phi^{+}$and a differential equation that contains only $\psi$ and $\phi$. A multiple-grid approach, in which a coarser mesh is used for $\psi$ than for $\phi$ and $\phi^{+}$in those-regions where $\psi$ varies more slowly than $\phi$ and $\phi^{+}$, is investigated. When the multiple grid is used, it is found that contributon flux distortions, which are indicative of inconsistencies among the difference equations, are introduced into the converged solutions. The problem of consistency is studied in detall for the case of slab geometry. The final approach involves writing a linear integral equation for a generalized contributon flux. However, this approach has not been actively pursued in detail.

\section{INTRODUCTION}

Linear neutral particle transport ${ }^{1}$ can be de.scribed by efther the forward Boltzmann equation,

$$
L \phi=Q,
$$

or the adjoint Boltzmann equation,

$$
\mathbf{L}^{+} \phi^{+}=\mathbf{R} \text {. }
$$

In these equations $\phi$ is the forward angular flux, $\phi^{+}$is the adjoint angular flux, $Q$ is the forward source distribution, $R$ is the detector response function and adjoint source, $L$ is the forward transpor operator, and $\mathrm{L}^{+}$is the adjoint transport operator defined such that

$$
\left\langle\phi^{+}, \mathrm{L} \phi\right\rangle=\left\langle\mathrm{L}^{+} \phi^{+}, \phi\right\rangle .
$$


The two descriptions are equivalent in the sense that any Integral response is given by

$$
I=\langle R, \phi\rangle=\left\langle Q, \phi^{+}\right\rangle,
$$

where $<,>$ Indicates an Integral over all phase space,

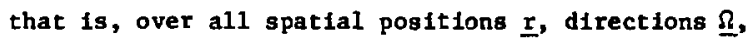
and energies $E$.

The standard approach to obtaining I Involves solving elther Eq. (I) or Eq. (2) and then using the appropriate integral in Eq. (4). However, a new approach, namely the contributon approach, has recently been suggested. 2,3 In this approach a contributon angular flux is defined as

$$
\psi(\underline{\underline{r}} \underline{\Omega}, E)=\phi(\underline{\mathbf{r}}, \underline{\Omega}, E) \phi^{+}(\underline{\mathbf{r}}, \underline{\Omega}, E)
$$

and the integral response is calculated from

$$
I=\iiint_{S_{Q}}\left(\hat{\mathbb{R}}_{Q} \cdot \underline{\Omega}\right) \psi(\underline{r}, \underline{\Omega}, E) d E d \underline{\Omega} d S
$$

or

$$
I=-\int_{S_{R}} \iint\left(\hat{\mathrm{n}}_{\mathrm{R}} \cdot \underline{\Omega}\right) \psi(\underline{\underline{r}}, \underline{\Omega}, \mathrm{E}) \mathrm{dEd} \underline{\underline{\Omega} \mathrm{dS}},
$$

where $S_{Q}$ is any surface that encloses the source but not the detector, $S_{R}$ is any surface that encloses the detector but not the source, and $\hat{n}_{Q}$ and $\hat{n}_{R}$ are the outward-directed untt noraal vectors on the surfaces, $S_{Q}$ and $S_{R}$, respectively. If surfaces $S_{Q}$ (or $S_{R}$ ) are chosen such that they are deacribed by the same unit normal vector $\hat{n}_{Q}$ (or $\hat{n}_{R}$ ), the wurface integrals In Eqs. (5a) and (5b) can be replaced by a volume integral. 4 For example, in slab geometry i could be calculated from

$$
I=\frac{2 \pi}{x_{2}-x_{1}} \int_{x_{1}}^{x_{2}} \int_{-1}^{1} \int_{\mu} \mu(x, \mu, E) d E d \mu d x
$$

where

$$
\left.\mu=\hat{n}_{Q} \cdot \underline{\Omega} \text { (or }-\hat{n}_{R} \cdot \underline{\Omega}\right)
$$

and the region, $x_{1}<x<x_{2}$, lien between the source and detector. This function $\psi$ provides not only suffictent information to calculate $I$ but also information on transport paths, in that peaks in $\psi$ correspond to preferred transport paths in phase space between the source and detector. This interpretation of $\psi$ is possible because contributons constitute the subset of source particles which actually reach the detector and contribute to the response, and 1 finds applications in channel theory analysis as described in Ref. 3.

One approach which has been used to conpute $\psi$ is to separately calculate $\phi$ and $\phi^{+}$and then to use the definition of $\psi$ in Eq. (5). ${ }^{3}$ The other approach, 2 however, involves solving for $\psi$ directly and is the approach documented in this report. In deep-penetration Konte Carlo calculations, where considerable effort is expended in tracking source particles which never reach the detector, the contributon approach has produced a significant reduction in effort by reducing the number of wasted historles. 4 In the deterministic approach, the goal is the reduction in effort which might be obtained from solving for one unknown, $\psi$, rather than two unknowns, $\phi$ and $\phi^{+}$. In addition, $\psi$ is expected to vary more slowly than $\phi$ and $\phi^{+}$over wuch of the system, so coarse weshes might be employed in those reglons where this is true. This slow variation is a direct consequence of the conservation law for $\psi$ derived in Ref. 2 .

Two approaches to the solution of the contributon problem are presented in Ref. 2. One Involves the solution of a linear complex differential equation in the unknown

$$
\Psi=\phi+1 \phi^{+}
$$

but this obviously produces no reduction in effort. The second approach involves the solution of a nonlinear real differential equation in $\psi$. This equathor was obtained for the special case of a pure absorber with constant cross section, source, and detector reaponse. However, no boundary conditions were specified and no atterpt was ande to solve this nonlinear equation.

The present report describes the physics of contributon transport and sone of the approaches conaidered for the deterainistic solution of the contributon 
transport problem. In the next section of this report, a contributon transport equation, Identical in form to the Boltzmann equation, is derived. From this equation, considerable Insight into contributon transport is gained. The derivation of this transport equation requires the introduction of a special contributon scattering cross section, and the properties of this cross section are described in the third section of this report. The fourth section contains detalled descriptions of four approaches to the solution of the contributon transport problem. The final section presents conclusions and recommendations for the contributon approach in general. provides: additional lnsight into the nature of the contributon transport problem.

\section{THE CONTRIBUTON TRANSPORT EQUATION}

In this section a transport equation for contributons will be derived. In so doing, new cross sections which describe the scattering and absorption of contributons will be introduced. This new transport equation will then be interpreted, and general boundary conditions for the contributon flux will be discussed.

A. Derivation of the Contributon Transport Equation In deriving the contributon transport equation, we begin with the steady-state forward Boltzmann equation,

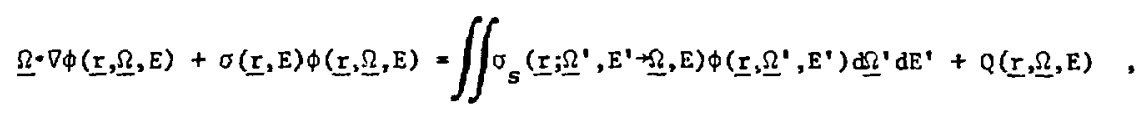

and the steady-state adjoint Boltzmann equation,

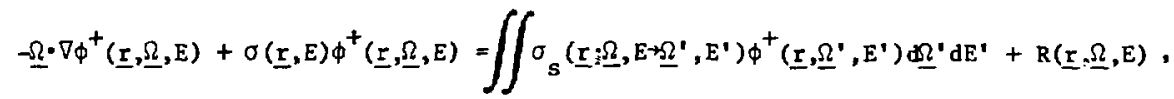

A brief description of the four solution approaches will now be presented. The first involves solving a quasi-linear third-order differential equation for $\psi$. This equation is a reformulation of the transport process described by the forward, adjoint, and contributon transport equations. The second approach involves solving a nonlinear differential equation for $\psi$. This equation is just a generalization of the nonlinear equation described In Ref. 2 . The third approach involves iterating betweet transformed forward and adjoint Boltzmann equations. These two equations have been transformed to take advantage of the slow variation of certain functions. A consistency analysis, which proved to be necessary for this approach, ylelded a set of consistent discrete-ordinates balance equations and supplementary equations for $\phi, \phi^{+}$, and $\psi$. The supplementary equations are a generalization of the exponentlal supplementary equations of Barbuccl and DiPasquantonio. The final approach Involves solution of an integral equation for a generalized contributon flux and where $\sigma(\underline{r}, E)=$ macroscopic total cross section, $\sigma_{s}\left(\underline{\underline{j}} \underline{\Omega}^{\prime}, E^{\prime}+\underline{\Omega}, E\right)=$ macroscopic differential scattering cross section, and the remaining quantities have been previously defined. These two equations are combined In the usual manner by multiplying Eq. (7) by $\phi^{+}$and Eq. (8) by $\phi$ and subtracting the second result from the first to obtain

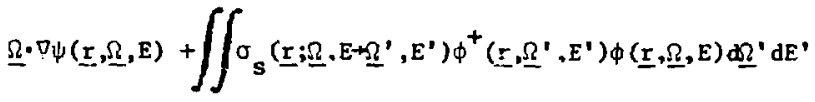

$$
\begin{aligned}
& =\iint \sigma_{s}\left(\underline{\underline{r}} ; \underline{\Omega}^{\prime}, E^{\prime}+\underline{\Omega}, E\right) \phi\left(\underline{r}, \underline{\Omega^{\prime}} E^{\prime}\right) \phi^{+}(r, \underline{\Omega}, E) d \underline{\Omega}^{\prime} \mathrm{d} E^{\prime} \\
& +Q(\underline{r}, \underline{\Omega}, E) \phi^{+}(\underline{\underline{r}}, \underline{\Omega}, E)-R(\underline{\underline{r}}, \underline{\Omega}, E) \phi(\underline{\underline{r}}, \underline{\Omega}, E) \text {. }
\end{aligned}
$$

At this polnt, a contributon differential "scattering" cross section, defined to be

$$
\bar{\sigma}_{s}\left(\underline{\underline{r}} ; \underline{\Omega}^{+}, E^{\prime}+\underline{\Omega}, E\right)=\sigma_{s}\left(\underline{\underline{r}} ; \underline{\Omega}^{\prime} E^{\prime}-\underline{\Omega}, E\right) \frac{\phi^{+}(\underline{\underline{r}}, \underline{\Omega}, E)}{\phi^{+} \underline{\left.\underline{r}, \underline{\Omega^{\prime}}, E^{\prime}\right)}},
$$

is introduced. With this definition, the two scatfering Integrals becone 


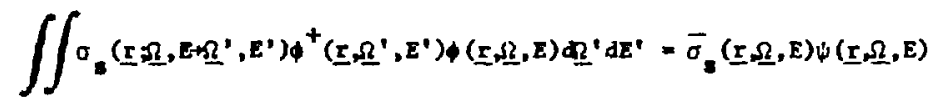

and

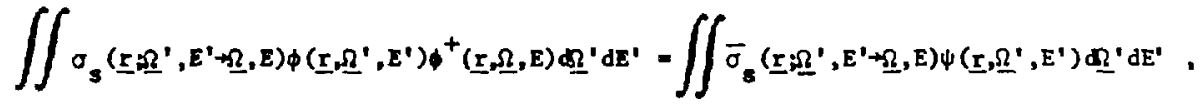

where the contributon scattering croms section $\bar{\sigma}_{g}(\underline{r}, \underline{\Omega}, E)$ is

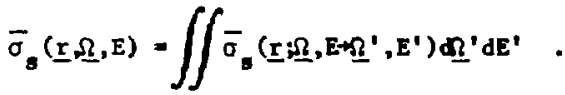

The cross section $\bar{\sigma}_{s}(\underline{r}, \Omega, E)$ becowes a function of $\underline{\Omega}$ since $\phi^{+}(\underline{r}, \underline{\Omega}, E)$ and $\iint \sigma_{s}\left(\underline{\underline{r}} ; \underline{\underline{\Omega}}, E^{-} \underline{\underline{\Omega}^{\prime}}, E^{\prime}\right) \phi^{+}\left(\underline{\underline{r}}, \underline{\Omega}^{\prime}, E^{\prime}\right)$ - $\Omega^{\prime} d E^{\prime}$ will not, in generel, have the sare functional dependence on $\underline{\Omega}$. This treatment of $\bar{\sigma}_{s}(\underline{\underline{r}}, \underline{\Omega}, E)$ as a direction dependent function is supported by numerical experiments, which will be described in detall in another section.

It is also possible to define contributon "absorption" and "total" cross sections. The contributon absorption cross section is defined to be

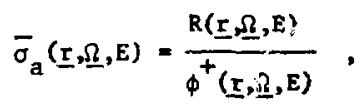

and the contributon total cross section is defined to be

$$
\bar{\sigma}(\underline{r}, \underline{\Omega}, E)=\bar{\sigma}_{s}(\underline{r}, \underline{\Omega}, E)+\bar{\sigma}_{a}(\underline{r}, \underline{\Omega}, E) .
$$

The definition (14) of the contributon absorption cross section 1s consistent with the definition of contributons as the subset of source particles which contribute to the detector response. Since contributons must contribute to the response, they can be removed from the system only by absorption in the detector.

When Eqs. (11)-(15) are used in Eq. (9), the contributon transport equation,

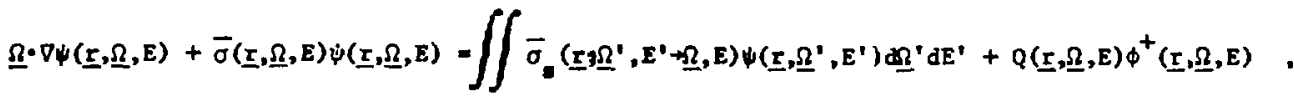

of the corresponding terms are similar for the two equations. However, in interpreting Eq. (16) in a Boltzmann-like manner, it must be remembered that the contributon is a fictitious particle. Unlike the neutron, each contributon has units associazed with it, namely, the units of the expected contribution of the contributon to the detector response. The result is that the contributon flux is not purely a particle flux. For example, if the integral response $I$ is a dose rate in units of rem/s, then the contributon flux can be vlewed as a dose flux with the units of rem/ $\mathrm{cm}^{2}$ - s.. sr - Mev.

Thus far nothing has been said to justify the Interpretation of $\bar{\sigma}_{s}$ as a scattering cross section. From Eq. (12), the integral term in the contributon transport equation is formally the source of contributons due to the scattering of forward particles. However, the formal identity between the contributon transport Eq. (16) and the forward Boltzmann equation might at this point be justification enough for in terpreting $\bar{\sigma}_{s}$ as a scattering cross section. If $\bar{\sigma}_{\mathrm{s}}$ Is so Interpreted, then the integral in Eq. (16) can be interpreted as the source of contributons due to the scattering of contributons. As a final note, with $\bar{\sigma}_{s}$ as the contributon scattering cross section, the contributon transport problem reduces to a pure scattering problem outside the detector region, because in regions where $R=0$, the contributon absorption cross section, as defined in Eq. (14), vanishes, and the contributon total cross section, as defined in Eq. (15), reduces to the total contributon scattering cross section; that is results. Thid equation is identical in form to the forward Boltzann equation, and the interpretations 


$$
\bar{\sigma}(\underline{r}, \underline{\Omega}, E)=\bar{\sigma}_{s}(\underline{r}, \underline{\Omega}, E)
$$

whenever $R(\underline{I}, \underline{\Omega}, E)$ is zero.

B. General Boundary Conditiong for the Contributon Flux

For problems with no purely absorbing regions,

all the boundary conditions for $\psi$ can be determined directly from the boundary conditions for $\phi$ and $\phi^{+}$. When purely absorbing regions are present, however, it appears that, except for certain special cases, at 3 east one of the boundary conditions for $\psi$ must be obtained by solving the forward or adjoint Boltzmarn equation in the pure absorber regions. For the present, only those boundary conditions which can be easily obtained from the boundary conditions on $\phi$ and $\phi^{+}$will be considered.

Consider first the boundary conditions at a nonreentrant vacuum boundary. If there is no neutron source outside the boundary, then for all posftions $r_{b}$ on the boundary surface,

$$
\phi\left(\underline{r}_{b}, \underline{\Omega}, E\right)=0 \text { if } \hat{n} \cdot \underline{\Omega}<0 \text {, }
$$

where $\hat{n}$ is the outward-directed unit normal on the boundary surface. If there is no detector outside the boundary, then for all positions $\underline{r}_{b}$ on the boundary surface,

$$
\phi^{+}\left(\underline{r}_{b}, \underline{\Omega}, E\right)=0 \text { if } \hat{n} \cdot \underline{\Omega}>0 \text {. }
$$

Thus, the boundary condition on $\psi$ for every position $\underline{r}_{b}$ on the boundary surface is just

$$
\psi(\underline{\underline{s}}, \underline{\Omega}, E)=0 \text { for all } \underline{\Omega} .
$$

The direction $\underline{\Omega}$ for which $\hat{\mathrm{n}} \cdot \underline{\Omega}=0$ is included in Eq. (20) even for a planar surface since

$$
\underset{\hat{n} \cdot \underline{\Omega \rightarrow 0^{+}}}{\lim } \psi\left(\underline{r}_{b}, \underline{\Omega}, E\right)=\underset{\hat{n} \cdot \Omega \rightarrow 0^{-}}{i 1 m} \psi\left(\underline{r}_{b}, \underline{\Omega}, E\right)=0 .
$$

Consider now an internal material interface where $\phi(\underline{\underline{r}}, \underline{\Omega}, \mathrm{E})$ and $\phi^{+}(\underline{\underline{r}}, \underline{\Omega}, \mathbf{E})$ are continuous in $\underline{\underline{r}}$, except possibly at a planar interface in those directions $\underline{\Omega}$ for which $\hat{n} \cdot \Omega=0$. The boundary condition on $\psi$ at a material interface is then $\psi(\underline{I}, \underline{\Omega}, \vec{z})$

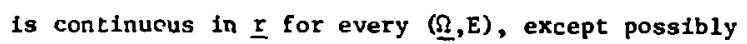

at a planar interface in those directions $\Omega$ for $\operatorname{tich} \hat{n} \cdot \underline{\Omega}=0$.

Finally consider the case of a reflecting boundary, for which

$$
\phi\left(\underline{r}, \underline{\Omega}_{1}, E\right)=\phi\left(\underline{r}_{2} \underline{\Omega}_{2}, E\right)
$$

and

$$
\phi^{+}\left(\underline{\underline{r}}, \underline{\Omega}_{1}, E\right)=\phi^{+}\left(\underline{\underline{r}}, \underline{\Omega}_{2}, E\right),
$$

where $\hat{n} \cdot \Omega_{1}=-\hat{n} \cdot \Omega_{2}$ and $\underline{\Omega}_{1} \neq \underline{\Omega}_{2}$. Then the boundary condition on $\psi$ at such a boundary is

$$
\psi\left(\underline{\underline{r}} \underline{\Omega}_{1}, E\right)=\psi\left(\underline{r}, \underline{\Omega}_{2}, E\right)
$$

In Sec. IV, other boundary conditions on $\psi$ constructed frow boundary conditions on $\phi$ and $\phi^{+}$will be encountered in connection with particular solution techniques. However, before possible techniques for solving the contributon problem are considered, the contriburion scattering cross section will be examined In more detail.

III. THE CONTRIBUTON SCATTERING CROSS SECTION In the previous chapter, several contributon cross sections were introduced to aid in the description of the transport of contributons. The definitions of these cross sections will now be used to determine some of the physics of contributon transport. Then an integro-differential equation for the contributon scattering cross 't tion will be derived, and some approximate solution, to this equation will be obtalned in slab geometry.

A. The Physics of Contributon Transport

The formal Identity between the contributon transport equation [Eq. (16)] and the forward Boltzmann equation [Eq. (7)] implies that contributons, like neutrons, 1 travel in straight lines between interactions with the medium. According to Eq. (15) there are only two types of contributon interactions, scatcering and absorption. From the definition of the contributon absorption cross section In Eq. (14), It can be seen that contributons are absorbed only in the detector. 
From the previous chapter, the definition of the differential cross section for contributon scatcering is

$\bar{\sigma}_{s}\left(\underline{\underline{r}} ; \underline{\Omega}^{\prime}, E^{\prime}+\underline{\Omega}, E\right)=\sigma_{s}\left(\underline{\underline{r}} ; \underline{\Omega}^{\prime}, E^{\prime}+\underline{\Omega}, E\right) \frac{\phi^{+}(\underline{\underline{r}}, \underline{\Omega}, E)}{\phi^{+}\left(\underline{\underline{r}}, \underline{\Omega}, E^{\prime}\right)}$

The ratio $\phi^{+}(\underline{r}, \underline{\Omega}, E) / \phi^{+}\left(\underline{r}, \Omega^{\prime}, E^{\prime}\right)$ in this definition Implies that contributons are preferentially scattered from directions $\Omega^{\prime}$ and energies $E^{\prime}$ of less importance to directions $\underline{\Omega}$ and energles $E$ of greater relative importance.

In addition, $\bar{\sigma}_{s}\left(\underline{\underline{I}} ; \underline{\Omega}^{\prime} E^{\prime}+\underline{\Omega}, E\right)$ can be infintce when $\phi^{+}\left(\underline{r} \underline{\Omega}^{\prime}, E^{\prime}\right)$ is zero. Since an infinite $\bar{\sigma}_{s}$ corresponds to a zero mean-free path for scattering, any surface on which $\phi^{+}\left(\underline{r}, \underline{\Omega}^{\prime}, E^{\prime}\right)$ is zero acts as a perfect reflector of contributons. A nonreentrant vacuum boundary is a prine example of such a surface. Since $\phi^{+}\left(\underline{r}, \underline{\Omega}^{\prime}, E^{\prime}\right)$ is zero for outgoing directions $\Omega^{\prime}\left(\hat{n} \cdot \Omega^{\prime}>0\right)$ an a vacuum boundary, contributons are effectively prevented from leaving the syaten. However, despite the fact that $\bar{\sigma}_{s}$ becowes infinite, it can be seen from Eqs. (11) and (12) that

$$
\bar{\sigma}_{s}(\underline{r}, \underline{\Omega}, E) \psi(\underline{r}, \underline{\Omega}, E) \text { and } \iint \bar{v}_{s}\left(\underline{r} ; \underline{j}^{\prime}, E^{\prime}+\underline{\Omega}, E\right) \psi\left(\underline{r}, \underline{\Omega}^{\prime}, E^{\prime}\right) \underline{\Omega}^{\prime} d E^{\prime}
$$

are bounded if $\phi$ and $\phi^{+}$are bounded.

One inal coment is in order concerning the contributon scattering cross section. It should be noted that the biasing,

$$
\frac{\phi^{+}(\underline{\underline{I}}, \underline{\Omega}, E)}{\phi^{+}\left(\underline{\underline{I}} \underline{\Omega}^{\prime}, E^{\prime}\right)}
$$

of the real scattering cross section $\sigma_{s}$ in Eg. (25) is similar to the zero varlance biasing in wonte Carlo importance sappling techniques. 6 Th1s suggests a possible equivalence between the contribution method and Monte Carlo Importance sampling. ${ }^{7}$

B. An Integro-Difierential Equation for the Contributon Scattering Cross Section

In the following analyols, only the case of monoenergetic transport in slab geometry with leotropic acattering is considered. The adjoint transport equation is first reuritcen by the use of Eqs. (10) and (13) to give $-\mu \frac{\partial \phi^{+}(x, \mu)}{\partial x}+\left[\sigma(x)-\bar{\sigma}_{s}(x, \mu)\right] \phi^{+}(x, \mu)=R(x, \mu)$.

For Isotroplc scattering, Eq. (11) ylelds

$$
\begin{aligned}
\vec{\sigma}_{s}(x, \mu) \phi^{+}(x, \mu) & =2 \pi \int_{-1}^{1} \frac{\sigma_{s 0}(x)}{4 \pi} \phi^{+}\left(x, \mu^{\prime}\right) d \mu^{\prime} \\
& =\sigma_{s 0}(x) \phi_{0}^{+}(x),
\end{aligned}
$$

where we chose

$$
\sigma_{s}\left(x ; \mu+\mu^{\prime}\right)=\frac{\sigma_{s 0}(x)}{4 \pi}
$$

ancं

$$
\phi_{0}^{+}(x)=\frac{1}{2} \int_{-1}^{1} \phi^{+}\left(x, \mu^{\prime}\right) d \mu^{\prime}
$$

Thus, from Eq. (27) we obtain

$$
\phi^{+}(x, \mu)=\frac{\sigma_{s o}(x) \phi_{0}^{+}(x)}{\sigma_{s}(x, \mu)},
$$

and from inserting Eq. (30) into Eq. (25) we obtain

$$
\bar{\sigma}_{s}\left(x ; \mu^{\prime}+\mu\right)=\frac{\sigma_{s 0}(x)}{4 \pi} \frac{\bar{\sigma}_{s}\left(x, \mu^{\prime}\right)}{\bar{\sigma}_{s}(x, \mu)} .
$$

Consequently, if scatering is isotropic, the differential contributon scattering cross section can be evaluated once $\bar{\sigma}_{\mathrm{s}}(x, \mu)$ is known, and $\bar{\sigma}_{\mathbf{s}}(x, \mu)$ can be computed through Eq. (27) if a solution for the adjoint flux is avallable.

If $R(x, \mu)=0$, Eq. (26) can be rewritten as 


$$
\begin{aligned}
\sigma(x)-\bar{\sigma}_{s}(x, \mu) & =\mu \frac{\partial}{\partial x}[\ln \stackrel{\phi}{\phi}(x, \mu)]^{+} \\
& =\mu \frac{\partial}{\partial x} \ln \left[\frac{\sigma_{s o}(x) \phi_{0}^{+}(x)}{\bar{\sigma}_{s}(x, \mu)}\right] \\
& =\mu \frac{d}{d x}\left[\ln \phi_{0}^{+}(x)\right]-\mu \frac{\partial}{\partial x}\left[\ln \bar{\Sigma}_{s}(x, \mu)\right],
\end{aligned}
$$

where

$$
\bar{\Sigma}_{s}(x, \mu)=\frac{\bar{\sigma}_{s}(x, \mu)}{\sigma_{s o}(x)}
$$

and where Eq. (27) has been utilized. Combining the adjoint equation [Eq. (26)] with Eq. (30), we obtain for $R=0$ the result,

$$
\begin{aligned}
& \frac{\partial \phi^{+}(x, \mu)}{\partial x}=\left[\frac{\sigma(x)-\bar{\sigma}_{\mathbf{g}}(x, \mu)}{\mu}\right] \phi^{+}(x, \mu) \\
& =\left[\frac{\sigma(x)-\bar{\sigma}_{s}(x, \mu)}{\mu \bar{\sigma}_{s}(x, \mu)}\right] \sigma_{s o}(x) \phi_{0}^{+}(x) \\
& =\left[\frac{\frac{a(x)}{s o}-\bar{\Sigma}_{s}(x, \mu)}{\mu \bar{\Sigma}_{s}(x, \mu)}\right] \sigma_{s o}(x) \phi_{0}^{+}(x),
\end{aligned}
$$

and after integrating over all directions $\mu$, we obtain

$\frac{d}{d x}\left[\ln \phi_{0}^{+}(x)\right]=\sigma_{s o}(x) \frac{1}{2} \int_{-1}^{1} \frac{\frac{\sigma(x)}{\sigma_{s o}(x)}-\bar{\Sigma}_{s}\left(x, \mu^{\prime}\right)}{\mu^{\prime} \bar{\Sigma}_{s}\left(x, \mu^{\prime}\right)} d \mu^{\prime}$.

If Eq. (35) is used to eliminate ${ }_{0}^{+}(x)$ from $\mathrm{Eq}$. (32), we obtain

$$
\begin{aligned}
\mu \frac{\partial}{\partial x}\left[\ln \bar{\Sigma}_{s}(x, \mu)\right]=\sigma_{s o}(x)\left[\bar{\Sigma}_{s}(x, \mu)-\frac{\sigma(x)}{\sigma_{s 0}(x)}+u \frac{1}{2} \int_{1}^{1} \frac{\frac{\sigma(x)}{\sigma_{s o}(x)}-\bar{\Sigma}_{s}\left(x, \mu^{\prime}\right)}{\mu^{\prime} \bar{\Sigma}_{s}\left(x, \mu^{\prime}\right)} d \mu^{\prime}\right] \\
\quad=\sigma_{s o}(x)\left\{\bar{\Sigma}_{s}(x, \mu)-\frac{\sigma(x)}{\sigma_{s o}(x)}\left[1-\frac{\mu}{v(x)}\right]\right\} .
\end{aligned}
$$

where

$$
\frac{1}{\nu(x)}=\frac{1}{2} \frac{\sigma_{s 0}(x)}{\sigma(x)} \int_{1}^{1} \frac{\frac{\sigma(x)}{\sigma_{s 0}(x)}-\bar{\Sigma}_{g}\left(x, \mu^{\prime}\right)}{\mu^{\prime} \bar{\Sigma}_{s}\left(x, \mu^{\prime}\right)} d \mu^{\prime} .
$$

To relterate, Eq. (36) is an exact integro-differential equacion for $\bar{\Sigma}_{s}(x \psi)$ assuming isotropic neutron scattering and is valid only in those regions where $R(x \downarrow)=0$.

\section{c. Approximat ion for the Concributon Scattering} Cross Section

In order to determine additional properties of $\bar{\sigma}_{s}(x, \mu)$ or $\bar{E}_{s}(x, \mu)$, the ONETRAN code ${ }^{8}$ has been used to generate the necessary adjoint fluxes for the geometry shown in Fig. 1. A number of different $\mathrm{S}_{\mathrm{N}}$ orders $(2 \leq N \leq 16)$ and scattering-co-rotal ratios $\left(0.1 \leq \sigma_{\text {so }} / 0 \leq 1.0\right)$ were used. Figure 2 is a plot of $\vec{\sigma}_{s}(x, \mu)$ obrained from Eq. (27) for the $S_{2}$ approximation and a scattering-co-total ratio of 0.5. The most important observation is the near constancy of $\bar{\sigma}_{s}(x, i)$ in the spatial variable $x$ in the central region which lies outside the detector region and is more than one mean-free path from the vacuum boundaries. Another important observation is the backward dominance of $\bar{\sigma}_{3}(x, \mu)$ in the direcition variable $\mu$ outside the detector region. These two properties were also observed for the other $S_{\mathrm{N}}$ orders and scarterling-to-total ratios used, although slightly more curvature was observed in $\bar{\sigma}_{s}(x, \mu)$ for scatceringto-total ratios near one.

The near constancy of $\vec{\sigma}_{s}(x, \mu)$ in the central region is due to the near separability in $x$ and $\mu$ of $\phi^{+}(x, \mu)$ in this region. The backward dominance of $\vec{\sigma}_{s}(x, \mu)$ outeide the detector region can be explained in the following way. Contributons must reach the detector region in order to contribute to the response. Since contributons traveling in directions with $\mu<0$ are traveling away from the 


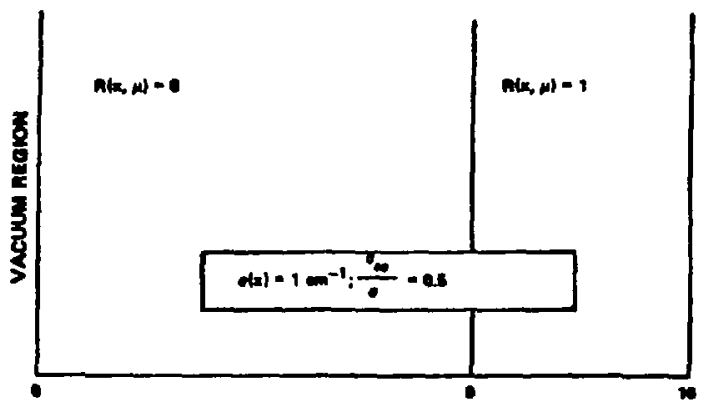

Patial position, not onami to scale (M.F.P.)

Fig. 1. Geometry for ONETRAN calculation of $\phi^{+}$.

toward the detector need not interact with the medium in order to reach the detector. This translates into a greater probability of contributon scattering for directions with $\mu<0$ than for directions with $\mu>0$ in this sapple problem.

The observation of the near constancy of $\bar{\sigma}_{s}(x, \mu)$ in the central region will now be used to obtain an approximation for $\vec{\sigma}_{s}(x, \mu)$ in that region. If $\bar{\sigma}_{s}(x, \mu)$ is assumed to be constant, then Eq. (36) in a region of uniform material properties [1.e., $\bar{\sigma}_{s}(x, \mu)=\bar{\sigma}_{s}(\mu), \sigma(x)=\sigma$, and $v(x)=v$ ] reduces to $\bar{\sigma}_{s}(\mu)=\sigma\left(1-\frac{\mu}{v}\right)$,

and Eq. (37) with the use of Eqs. (33) and (38) becomes

$$
\frac{\sigma \text { so }}{\sigma} \frac{v}{2} \int_{-1}^{1} \frac{\mathrm{d} \mu^{\prime}}{v-u^{\prime}}=1 \text {. }
$$

Thus, $v$ is just the discrete eigenvalue given by Case's method. 9 In fact, it can easily be shown that the constancy of $\bar{\sigma}_{s}(x, \mu)$ is equivalent to the adjoint flux being coopletely described by the dominant asymptotic efgenfunction; that is,

$$
\phi^{+}(x, \mu)-\frac{e^{\sigma x / v}}{1-\frac{1 !}{v}} .
$$

Since this dominance by one of the two asymptotic terms beromes less pronounced as the scattering-tototal ratio approaches one, $\bar{\sigma}_{s}(x, \mu)$ exhiblts wore curvature and the approxtmation given by $\mathrm{Eq}$. (38) becomes poorer as this ratio approaches one.

In the discrete-ordinates approxtmation, the integral in Eq. (39) is replaced by a discrete su, namely

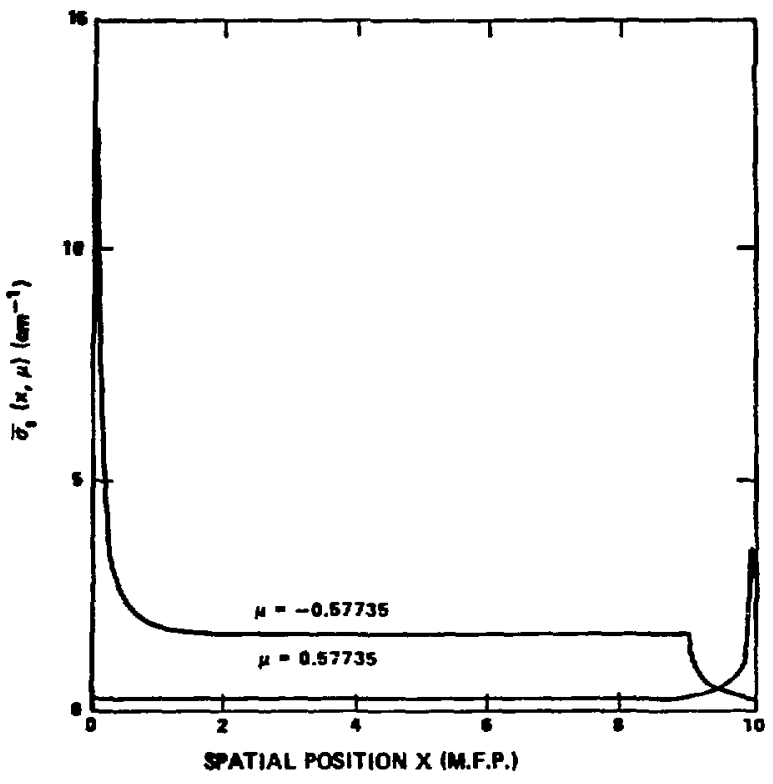

F1g. 2. $\bar{\sigma}_{s}(x, \mu)$ for the $s_{2}$ approximation and $\sigma_{\text {so }} / 0=0.5$.

$2 \frac{\sigma_{\text {so }}}{\sigma} \sum_{m=1}^{M / 2} \frac{\omega_{m}}{1-\left(\frac{\mu m}{v}\right)^{2}}=1 \quad\left(\max _{m}\left|\mu_{m}\right|<v<\infty\right)$,

where

$$
\begin{aligned}
& \sum_{m=1}^{M} w_{m}=1, \\
& M=\text { number of discrete directions, }
\end{aligned}
$$

and where the quadrature set is symetric. Figure 3 is a plot of $1 / V$ vs the scatcering-to-total ratio for the case of continuous $\mu$ and for the $S_{2}$ and $S_{4}$ approxiwations. Also show are data points for $1 / \mathrm{v}$ at the slab center based upon the previously described ONETRAN calculations. The agreement of the ONETRAN results with the approximation curves is very good for $\sigma_{s o} f \sigma<0.9$, but a deviation occurs for $\sigma_{\text {so }} / \sigma>$ 0.9 , as expected from the foregoing discusston. 
IV. APPROACHES TO THE SOLUTION OF THE CONTRIBUTON TRANSPORT PROBLEM

Four different approaches to solving the contributon problem are considered. The first involves the solution of a quasi-linear differential equation in the unknown $\psi(\underline{r}, \Omega, E)$. This equation is obtained by eliminating $\phi$ and $\phi^{+}$from the contributon balance equation. The second approach involves the solution of a nonlinear differential equation in the unknown $\psi(\underline{\underline{r}} \underline{\Omega}, E)$. The third approach involves iterating between a transformed forward Boltzmann equation and a transformed adjoint Boltzmann equation. These equations have been transformed to exploit the slow variation of certain functions. The final approach involves solving an integral equation for a generalized contributon flux to be defined later. Only the first and third approaches have been actively pursued in detail, but the others also provide some insight into the contributon transport problem. A. Quasi-linear Differential Equation For $\psi$

This approach involves eliminating explicit occurrences of $\phi$ and $\phi^{+}$from the contributon transport equation to obtain a differential equation in the unknown $\psi(\underline{r}, \underline{\Omega}, E)$. The order of the resulting equation depends upon the characteristics of the forward source,

$$
\begin{aligned}
& S(\underline{\underline{r}}, \underline{\Omega}, E)=\iint \sigma_{s}\left(\underline{I} ; \underline{\Omega}^{\prime}, E^{\prime}+\underline{\Omega}, E\right) \phi\left(\underline{\underline{r}}, \underline{\Omega}^{\prime}, E^{\prime}\right) \mathrm{d} \underline{\underline{\Omega}} \underline{\underline{\Omega}}^{\prime} \mathrm{dE} \\
& +Q(\underline{\underline{r}}, \underline{\Omega}, \mathrm{E}),
\end{aligned}
$$

and the adjoint source,

$$
\begin{aligned}
& S^{+}(\underline{r}, \underline{\Omega}, E)=\iint \sigma_{s}\left(\underline{r} ; \underline{\Omega}, E+\underline{\Omega} \underline{\Omega}^{\prime}, E^{\prime}\right) \phi^{+}\left(\underline{r}, \underline{\Omega}^{\prime}, E^{\prime}\right) d \underline{\Omega^{\prime}} \mathrm{d} E^{\prime} \\
& \quad+\mathrm{R}(\underline{r}, \underline{\Omega}, E) .
\end{aligned}
$$

Therefore, the following region designations are introduced:

(1) 0 region, in which $s(\underline{r}, \underline{\Omega}, E) \equiv 0$ and $s^{+}(\underline{r}, \underline{\Omega}, E) \equiv 0$,

(2) $\sum$ region, in which $S(\underline{r}, \underline{\Omega}, E) \neq 0$ and $s^{+}(\underline{r}, \underline{\Omega}, E) \equiv 0$,

(3) $\Sigma^{+}$region, in which $S(\underline{\underline{r}}, \underline{\Omega}, E) \equiv 0$ and $S^{+}(\underline{\underline{r}}, \underline{\Omega}, E) \neq 0$, and

(4) $\Sigma \Sigma^{+}$region, in which $S(\underline{\underline{r}}, \underline{\Omega}, E) \neq 0$ and $S^{+}(\underline{\underline{r}}, \underline{\Omega}, E) \neq 0$.

In terms of $S$ and $S^{+}$, the forward and adjoint

Boltzmann equations and the contributon balance, or conservation, equation are

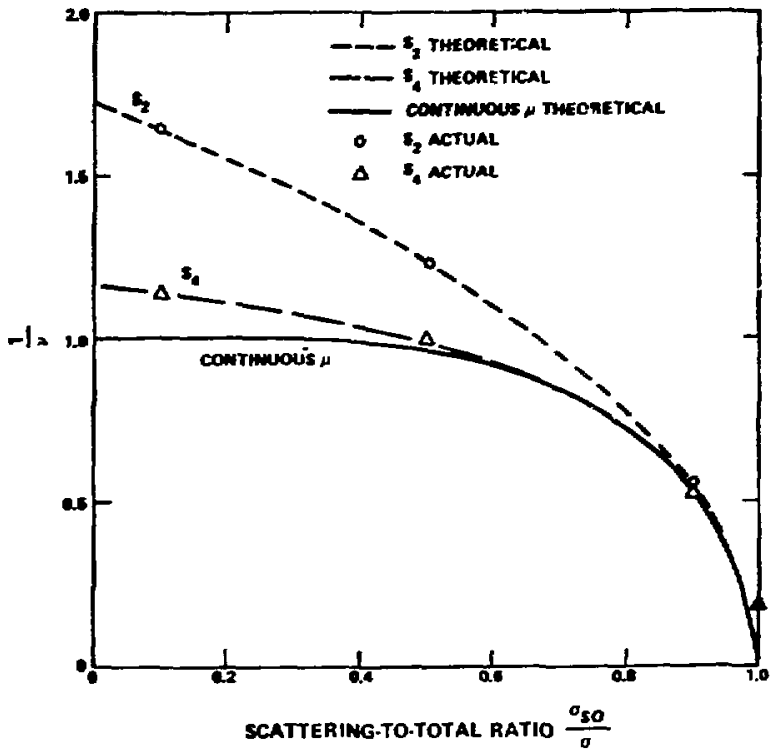

Fig. 3. 1/v vs scattering-to-tatal ratio.

$$
\begin{aligned}
& \Omega \cdot \nabla \phi+\sigma \phi=s, \\
& \underline{-\Omega} \cdot \nabla \phi^{+}+\sigma \phi^{+}=s^{+},
\end{aligned}
$$

and

$$
\underline{\Omega} \cdot \nabla \psi=\mathrm{S}^{+}-\mathrm{S}^{+} \phi
$$

Explicit occurrences of $\phi$ and $\phi^{+}$will now be eliminaced. Of course, $\phi$ and $\phi^{+}$still occur implicitly in $S$ and $\mathrm{S}^{+}$, and this is the reason why we call the resulting differential equations for $\downarrow$ "quasi-linear."

\section{Derfvation of Differential Equations for $\dot{\psi}$}

Consider first the contributon censervation equation [Eq. (46)]. Obviously, if both $s$ and $s^{+}$ are zero, both $\phi$ and $\phi^{+}$are eliminated. Consequently, the result is the first-order differential equa+ion,

$$
\underline{\Omega} \cdot \nabla \psi=0, \quad(\underline{r}, \underline{\Omega}, E) \varepsilon 0 .
$$

To determine when a second-order equation is suitable, $E_{q}$. (46) is first differentiated to give

$(\underline{\Omega} \cdot \nabla)^{2} \psi=\phi^{+} \underline{\Omega} \cdot \nabla s+\underline{s} \cdot \nabla \phi^{+}-\phi \underline{\underline{\Omega}} \cdot \nabla s^{+}-\dot{s} \underline{\Omega} \cdot \nabla \phi$.

A linear combination of ihis equation and Eqs. (44)(46) is formed to give 


$$
\begin{aligned}
& A(\Omega \cdot \nabla)^{2} \psi+B \underline{\Omega} \cdot \nabla \psi+C S+D S^{+}=\left(-A \Omega \cdot \nabla S^{+}-B S^{+}+C \sigma\right) \phi+(A \Omega \cdot \nabla S+B S+D \sigma) \phi^{+} \\
& \quad+\left(-A S^{+}+C\right) \underline{\Omega} \cdot \nabla \phi+(A S-D) \underline{\Omega} \cdot \nabla \phi^{+},
\end{aligned}
$$

where the quantities $A, B, C$, and $D$ are to be determined so that the coeffirients of $\phi, \phi^{+}, \Omega \cdot \nabla \phi$, and $\Omega \cdot \nabla \phi^{+}$are zero as a necessary condition that Eq. (49) reduces to an equation in $\psi$ alone. The following system of equations results:

$$
\left[\begin{array}{cccc}
-\Omega \cdot \nabla S^{+} & -S^{+} & \sigma & 0 \\
\Omega \cdot \sigma \sigma & S & 0 & 0 \\
-S^{+} & 0 & 1 & 0 \\
S & 0 & 0 & -1
\end{array}\right]\left[\begin{array}{l}
A \\
B \\
C \\
D
\end{array}\right]
$$

Since this system of equations [Eq. (50)] is homogeneous, a nontriviai solution exists only if the determinant $\Delta_{2}$ of the coefficient matrix is ze ro, that is,

$$
\Delta_{2}=\mathrm{S} \Omega \cdot \nabla S^{+}-s^{+} \underline{\Omega} \cdot \nabla S-2 \sigma S s^{+}=0 .
$$

$\Delta_{2}$ will be zeru if
(1) $s \equiv 0$,
(2) $s^{+} \equiv \hat{0}$,
(3) $s \equiv 0$ and $s^{+} \equiv 0$,

or

(4) $s \underline{\Omega} \cdot \nabla s^{+}-s^{+} \underline{\Omega} \cdot \nabla s-2 \sigma s s^{+}=0$ with $s \neq 0$ and $s^{+} \neq 0$.

The last condition is unlikely to be satisfied since $S, S^{+}$, and $\sigma$ are independent. If both $S$ and $s^{t}$ are zero, the first-order differential equation should be used. Thus, only the first two conditions need to be considered.

If the first condition is considered and $A$ is arbitrarily set to one, only three of the equations in the system of Eqs. (50) remain Independent. The residual system of independent equations,

$$
\left[\begin{array}{ccc}
-s^{+} & \sigma & 0 \\
0 & 1 & 0 \\
0 & 0 & -1
\end{array}\right]\left[\begin{array}{l}
B \\
C \\
D
\end{array}\right]=\left[\begin{array}{c}
\Omega \cdot \nabla s^{+} \\
s^{+} \\
0
\end{array}\right],
$$

is solved for $B, C$, and $D$, and the results are substituted into Eq. (49) to yield the second-order differential equation,

$$
\begin{gathered}
(\underline{\Omega} \cdot \nabla)^{2} \psi+\left(\sigma-\frac{1}{s^{+}} \underline{\Omega} \cdot \nabla s^{+}\right) \underline{\Omega} \cdot \nabla \psi=0, \\
(\underline{\underline{r}}, \underline{\Omega}, \mathrm{E}) \varepsilon \Sigma^{+} .
\end{gathered}
$$

If the second condition is considered and $A$ is arbitrarily set to one, the syster of independent equations,

$$
\left[\begin{array}{rrr}
\mathrm{s} & 0 & 0 \\
0 & 1 & 0 \\
0 & 0 & -1
\end{array}\right]\left[\begin{array}{l}
\mathrm{B} \\
\mathrm{C} \\
\mathrm{D}
\end{array}\right]=\left[\begin{array}{l}
-\frac{\Omega}{0} \cdot \nabla \mathrm{s} \\
0 \\
-\mathrm{S}
\end{array}\right],
$$

is obtained. If the resulis for $B, C C$, and $D$ from Eq. (54) are used in Eq. (49), the rasult is the second-order differential equation,

$$
\begin{gathered}
(\underline{\Omega} \cdot \nabla)^{2} \psi-\left(\sigma+\frac{1}{S} \underline{\Omega} \cdot \nabla S\right) \Omega \cdot \nabla \psi=0, \\
(\underline{\underline{I}}, \underline{\Omega}, \mathrm{E}) \varepsilon \Sigma .
\end{gathered}
$$

To obtain a third-order equation for $\psi$, Egs . (48), (44), and (45) are first differentiated to give

$$
\begin{aligned}
& (\Omega \cdot \nabla)^{3} \psi=\phi^{+}(\underline{\Omega} \cdot \nabla)^{2} s+2(\underline{\Omega} \cdot \nabla s) \underline{\Omega} \cdot \nabla \phi^{+}+s(\underline{\Omega} \cdot \nabla)^{2} \phi^{+} \\
& -\phi(\underline{\Omega} \cdot \nabla)^{2} s^{+}-2\left(\Omega \cdot \nabla S^{+}\right) \Omega \cdot \nabla \phi-s^{+}(\Omega \cdot \nabla)^{2} \phi, \\
& (\Omega \cdot \nabla)^{2} \phi+\sigma \Omega \cdot \nabla \phi+\phi \underline{\Omega} \cdot \nabla \sigma=\Omega \cdot \nabla s,
\end{aligned}
$$

and

$-(\underline{\Omega} \cdot \nabla)^{2} \phi^{+}+\sigma \underline{\Omega} \cdot \nabla \phi^{+}+\phi^{+} \underline{\Omega} \cdot \nabla \sigma=\underline{\Omega} \cdot \nabla \mathrm{S}^{+}$.

A linear combination of Eqs. (56)-(58), (48), and (44)-(46) is formed with arbitrary coeffictents A-G to give 


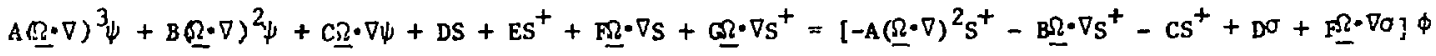

$$
\begin{aligned}
& +\left[A(\underline{\Omega} \cdot \nabla)^{2} S+B \underline{B} \cdot \nabla S+C S+E \sigma+\underline{G} \underline{\Omega} \cdot \nabla \sigma\right] \phi^{+}+\left(-2 \underline{A} \Omega \cdot \nabla S^{+}-B S^{+}+D+F \sigma\right) \Omega \cdot \nabla \phi+(2 A \Omega \cdot \nabla S+B S-E+G \sigma) \Omega \cdot \nabla \phi \\
& +\left(-\mathrm{AS}^{+}+\mathrm{F}\right)(\underline{\Omega} \cdot \nabla)^{2} \phi+(\mathrm{AS}-\mathrm{G}) \underline{(\Omega \cdot \nabla)^{2} \phi^{+}}
\end{aligned}
$$

If the quantities $A-G$ are chosen so that the coefficlents of $\phi, \phi^{+}, \underline{\Omega} \cdot \nabla \phi, \underline{\Omega} \cdot \nabla \phi^{+},(\underline{\Omega} \cdot \nabla)^{2} \phi$, and $(\underline{\Omega} \cdot \nabla)^{2} \phi^{+}$ are zero, we obtain a necessary condition for the existence of a third-order equation for $\psi$. If $A=1$, the following system of equations results:

$\left[\begin{array}{cccccc}-\Omega \cdot \nabla S^{+} & -S^{+} & \sigma & 0 & \underline{\Omega} \cdot \nabla \sigma & 0 \\ \Omega \cdot \nabla S & S & 0 & \sigma & 0 & \underline{\Omega} \cdot \nabla \sigma \\ -S^{+} & 0 & 1 & 0 & 0 & 0 \\ S & 0 & 0 & -1 & 0 & 0 \\ 0 & 0 & 0 & 0 & 1 & 0 \\ 0 & 0 & 0 & 0 & 0 & -1\end{array}\right]$

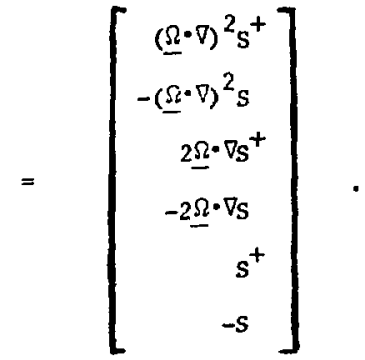

Comparing Eq. (60) with Eq. (50), we note that the determinant of the coefficient matrix of Eq. $\langle 60\rangle$ Is

$$
\Lambda_{3}=-\Delta_{2}
$$

but if $\Delta_{2}$ is zero, then the first- or second-order differential equation for $\psi$ results. Therefore, we consider only the case $\Delta_{2} \neq 0$, which will occur only in a $\Sigma \Sigma^{+}$region. The system of equations [Eq. (60)] can then be solved for the quantities $B-G$. If these results are then substituted into $\mathrm{Eq}$. (59), we obtain the third-order differential equation, equations [Eqs, (53) and (55)] are appropriate for pure absorber reglons where either $Q$ or $R$ is zero, and the first-crder equation [Eq. (47)] is appropriate for pure absorier regions where both $Q$ and $R$ are zero. Table I summarizes the conditions under which the first-order, second-order, or third-order quasilinear differential equations for $\psi$ derived in this section describe the contributon transport problem. In analogy to calling the Boltzmann equation a transport equation for neutral particles, we may

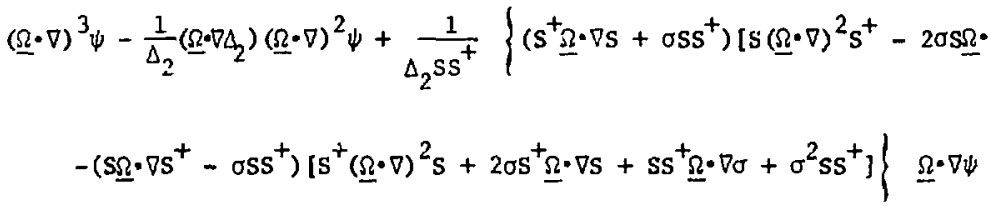

$$
\begin{aligned}
& +3 \underline{\Omega} \cdot \nabla\left(S S^{+}\right)-\frac{2}{\Delta_{2}} \mathrm{SS}^{+} \underline{\Omega} \cdot \nabla \Delta_{2}=0, \quad(\underline{\underline{I}}, \underline{\Omega}, \mathrm{E}) E \Sigma \Sigma^{+} .
\end{aligned}
$$

The third-order equation [Eq. (62)] must be used in pure absorber regions where both $Q$ and $R$ are nonzero and in scattering regtons, since both $S$ and $\mathrm{s}^{+}$ are nonzero in such regions. The second-order cal1 Eqs. (47), (53), (55) and (62) transport equations for contributons. 10 
TABLE I

CONDITIONS FOR APPROPRIATE FORMS OF

QUASI-LINEAR CONTRIBUTON TRANSPORT EQUATIONS

\begin{tabular}{|c|c|c|c|c|}
\hline & \multicolumn{4}{|c|}{ Transport Equation Form by Region Type } \\
\hline & $0\left(s \equiv 0, s^{+} \equiv 0\right)$ & $\Sigma\left(s \neq 0, s^{+} \equiv 0\right)$ & $\underline{\Sigma}^{+}\left(s \equiv 0, s^{+} \neq 0\right)$ & $\underline{\Sigma \Sigma^{+}}\left(s \neq 0, s^{+} \neq 0\right)$ \\
\hline Pure Absorber & 1 & $2_{B}$ & $2_{A}$ & 3 \\
\hline Scattering Medium & - & - & - & 3 \\
\hline
\end{tabular}

1 a first-order Eq. (47)

${ }_{\mathrm{A}} \cong$ second-oŕder Eq. (53)

$2_{B}$ a second-order Eq. (55)

3 今 third-order Eq. (62)

It will be shown in a later section that the first two integrations of Eq. (62) are equivalent to solving for $\phi$ and $\phi^{+}$from the Boltzmann equations and the final integration gives $\psi$. In other words, the three first-order equations [Eqs. (44)-(46)] have merely been replaced by the one third-order equatioti [Eq. (62)].

\section{Boundary Conditions for $\psi$}

In this section, only problems with external vacuum boundaries are considered. Obvious1y, for the third-order differential equation, three external boundary conditions are required. As it turns out, however, there are four to choose from; but only three are independent. According to Egg. (18)(20), two of these are given by

$$
\psi\left(\underline{\underline{r}}_{b}, \underline{\Omega}, E\right)=0 \text { for every }(\underline{\Omega}, E)
$$

on the vacuum boundary $\underline{r}=\underline{r}_{b}$.

Two additional boundary conditions for $\psi$ can be constructed in the following manner. Since the third-order differential equation for $\psi$ and derivatives of that equation are satisfied at every position, we seek a boundary condition on $\psi$ which involves derivatives of $\psi$ no higher than second order. A suitable starting point for deriving such a condition is provided by Eq. (49), namely
Since $\Delta_{3}$, and consequently $\Delta_{2}$, as defined by Eq. (51), must be nonzero if the third-order equation for $\psi$ is to be valid, we rannot solve for all four of the quantities $A-D$ as was done in deriving the second-order equations [Eqs. (53) and (55)]. We can, however, choose A-D so that either the coefficlents of $\phi$ and $\Omega \cdot \nabla \phi$ are zero or the coefficlents of $\phi^{+}$and $\Omega \cdot \nabla \phi^{+}$are zero.

If the coefficients of $\phi$ and $\underline{\Omega} \cdot \nabla \phi$ are required to be zero in Eq. (64) and if $A$ is arbitrarily set to one, then the system of aquations,

$$
\left[\begin{array}{cc}
-s^{+} & \sigma \\
0 & 1
\end{array}\right]\left[\begin{array}{l}
B \\
C
\end{array}\right]=\left[\begin{array}{c}
\underline{\Omega} \cdot \nabla s^{+} \\
s^{+}
\end{array}\right]
$$

results. If. Eq. (64a) is solved for $B$ and $C$ and the results are used in $\mathrm{Eq}$. (64), the result is

$$
\begin{aligned}
& \underline{(\Omega-\nabla)^{2}} \psi+\left(\sigma-\frac{1}{s^{+}} \underline{\Omega} \cdot \nabla s^{+}\right) \Omega \cdot \nabla \psi+s s^{+} \\
& =\left[\underline{\Omega} \cdot \nabla s+s\left(\sigma-\frac{1}{s^{+}} \underline{\Omega} \cdot \nabla s^{+}\right)\right] \phi^{+}+\underline{\Omega}-\nabla \phi^{+} \\
& =-\frac{\Delta_{2}}{s^{+}} \phi^{+}-s s^{+},
\end{aligned}
$$

$A(\Omega \cdot \nabla)^{2} \psi+B \Omega \cdot \nabla \psi+C S+D S^{+}=\left(-A Q \cdot \nabla S^{+}-B S^{+}+C \sigma\right) \phi+\left(-A S^{+}+C\right) \underline{\Omega} \cdot \nabla \phi+(A \Omega \cdot \nabla S+B S+D \sigma) \phi^{+}+(A S-D) \underline{\Omega} \cdot \nabla \phi^{+}$. 
or

$$
\underline{\Omega} \cdot \nabla)^{2} \psi+\left(\sigma-\frac{1}{s^{+}} \Omega \cdot \nabla s^{+}\right) \Omega \cdot \nabla \psi+2 s s^{+}=-\frac{\Delta}{s^{+}} \phi^{+} .
$$

Use of the vacuum boundary conditions for $\phi^{+}$then gives:

$$
\begin{aligned}
& (\underline{\Omega \cdot \nabla})^{2} \psi+\left(\sigma-\frac{1}{s^{+}} \underline{\Omega} \cdot \nabla s^{+}\right) \underline{\Omega} \cdot \nabla \psi+2 s s^{+}=0 \\
& \text { for } \hat{n} \cdot \underline{\Omega}>0
\end{aligned}
$$

on the vacuum boundary surface. If the coefficients of $\phi^{+}$and $\Omega \cdot \nabla \phi^{+}$are required to be zero, then a similar analysis using the boundary condttions for $\phi$ gives the boundary condition

$$
\begin{aligned}
& (\underline{\Omega} \cdot \nabla)^{2} \psi-\left(\sigma+\frac{1}{S} \underline{\Omega} \cdot \nabla S\right) \Omega \cdot \nabla \psi+2 S S^{+}=0 \\
& \text { for } \hat{n} \cdot \underline{\Omega}<0
\end{aligned}
$$

on the vacuum boundary surface.

One additional benefit of this analysis is a set of equations for $\phi$ and $\phi^{+}$in terms of $\psi$ for a $\Sigma \Sigma^{+}$region, namely

$\phi=-\frac{S}{\Delta_{2}}\left[(\underline{\Omega} \cdot \nabla)^{2} \psi-\left(\sigma+\frac{1}{S} \underline{\Omega} \cdot \nabla S\right) \underline{\Omega} \cdot \nabla \psi+2 S S^{4}\right]$

and

$\left.\phi^{+}=-\frac{s^{+}}{\Delta_{2}}[\underline{(\Omega} \cdot \nabla)^{2} \psi+\left(\sigma-\frac{1}{s^{+}} \underline{\Omega} \cdot \nabla s^{+}\right) \underline{\Omega} \cdot \nabla \psi+2 s s^{+}\right]$,

which are obtained from Eq. (66) and the corresponding equation that leads to Eq. (68). These two equations permit us to evaluate $\phi$ and $\phi^{+}$, and consequently $S$ and $S^{+}$, in an iterative solution of Eq. (62), as described at the end of Sec. IV.A.7. These results also allow us to specify the boundary conditions at internal material interfaces, namely that

$\psi(\underline{r}, \underline{\Omega}, E)$ is continuous in $\underline{r}$,

$\frac{S}{\Delta_{2}}\left[(\underline{\Omega} \cdot \nabla)^{2} \psi-\left(\sigma+\frac{1}{S} \underline{\Omega} \cdot \nabla S\right) \underline{\Omega} \cdot \nabla \psi+2 S S^{+}\right]$is continuous

in $\underline{r}$, and
$\frac{s^{+}}{\Delta_{2}}\left[(\Omega \cdot \nabla)^{2} \psi+\left(\sigma-\frac{1}{s^{+}} \Omega \cdot \nabla s^{+}\right) \Omega \cdot \nabla \psi+2 s s^{+}\right]$is continuous

in $\underline{x}$.

It should be noted that expressions for $\phi$ and $\phi^{+}$in terms of $\psi$ can be obtained for $\Sigma$ and $\Sigma^{+}$regions

from the contributon conservation equation [Eq. (46)]. When an 0 region exists, it is apparently impossible to completely specify the boundary conditions on $\psi$ without effectively solving the forward or adjoint Bolczmann equation in the 0 region. This difficulty is attributed specifically to the fact that only in an 0 region is $1 t$ apparently impossible to express $\phi$ and $\phi^{+}$in terms of $\psi$ and its derivatives. Consider, for example, the pure absorber problem shown in Fig. 4. The appropriate contributon transport equation for $\psi$ is second-order in the $\Sigma$ and $\Sigma^{+}$regions and first-order in the 0 region. Therefore, five boundary conditions are required for $\psi$. Two of these are the external vacuum boundary conditions,

$\psi(0, \mu)=\psi(c, \mu)=0 \quad$ for every $\mu$,

and two are the internal continuity condtions,

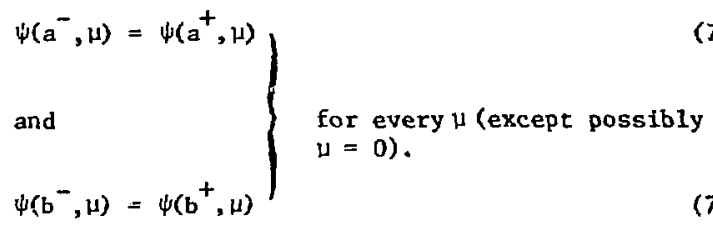

For the final condition, it appears that efther the forward or the adjoint Boltzmann equation must

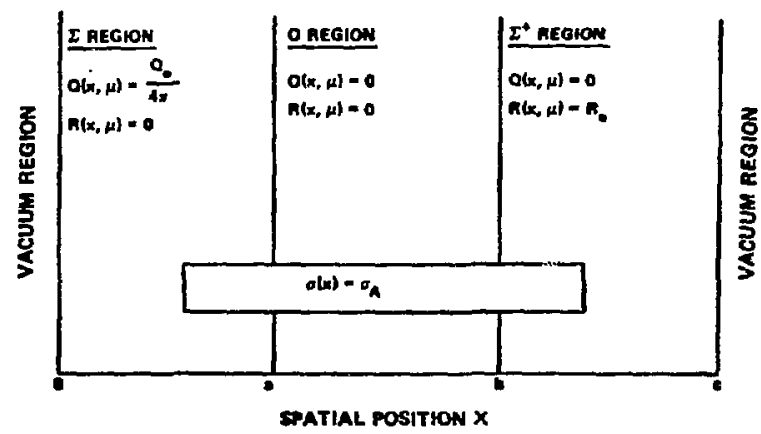

Fig. 4. Geometry for pure absorber problen 1. 
be solved in the 0 region when such a region exists. The necessity of obtaining $\phi$ or $\phi^{+}$in order to spectfy the boundary condition on $\psi$ has also been recognized by Gandini ${ }^{11}$ based on heuristic arguments. In the present formulation, we choose to solve for $\phi^{+}$. From the contributon balance equation [Eq. (46)] we obtain in slab geometry

$$
\mu \frac{\partial \psi\left(a^{-}, \mu\right)}{\partial x}=Q\left(a^{-}, \mu\right) \phi^{+}(a, \mu)
$$

and

$$
\mu \frac{\partial \psi\left(b^{+}, \mu\right)}{\partial x}=-R\left(b^{+}, \mu\right) \phi(b, \mu) .
$$

Solving the adjoint equation [Eq. (45)] in the 0 region yields

$$
\phi^{+}(a, \mu)=\phi^{+}(b, \mu) e^{-\sigma} A^{(b-a) / \mu} .
$$

Multiplying together corresponding sides of Eqs. (74) and (75) and using Eq. (76) give the fifth boundary condition for the pure absorber problem,

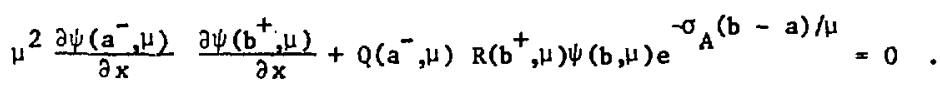

be solved for $\phi$ to give the resuli in Eq. $699^{\circ}$. If this expression for $\phi$ is then substituted ip to the forward Bolczmann equation [Eq. (44)] then the

$c n$ the other hand, the contributor balance equation [Eq. (46)] could be solved for $\phi$ and this expresston for $\phi$ then substituted into the forward Boltzmann equation to give, aftez use of the adjoint equation [Eq. (45)], tite result, $(\Omega \cdot \nabla)^{2} \psi+\left(\sigma-\frac{1}{s^{+}} \underline{\Omega} \cdot \nabla s^{+}\right) \Omega \cdot \nabla \psi+2 s^{+}=-\frac{\Delta_{2}}{s^{+}} \phi^{+}$.

This equation can be solved for $\phi^{+}$to give the result in Eq. (70), and if this expression for $\phi^{+}$ is then substituted finto the adjoint Boltzmann Eq. (45), the same third-order equation [Eq. (62)] results.

Although a single third-order differential equation for $\psi$ results whether it is obtained by third-order equation [Eq. (62)] results.
This is a nonlinear mixed boundary condition, which will be referred to as the bridging condition for obvious reasons.

\section{An Alternative Derivation of the Tnird- Order Equation for $\psi$}

Instead of abtaining the third-order equation for $\psi$ by forming a linear combination of a set of differential equations, another approach, which gives some useful insight, will now be taken. The contributon balance equation [Eq. (46)] is solved for $\phi^{+}$to give

$$
\phi^{+}=\frac{1}{s}\left(\Omega 2 \cdot \nabla \psi+s^{+} \phi\right)
$$

This result is subseltuted into the adjoint Boltzmann equation [Eq. (45)] to give, after use of the forward Boltzmann equation [Eq. (44)], the result,

$(\underline{\Omega} \cdot \nabla)^{2} \psi-\left(\sigma+\frac{1}{s} \underline{\Omega} \cdot \nabla S\right) \underline{\Omega} \cdot \nabla \psi+2 S s^{+}=-\frac{\Delta_{2}}{s} \phi$,

where $\Delta_{2}$ is defined in Eq. (51). This equation can inserting the expression for $\phi$ into the forward Boltzmann equation or by inserting the expression for $\phi^{+}$into the the adfoint Boltzmann equation, it appears that a single integration of this thirdorder equation cannot yield both $\phi$ and $\phi^{+}$. Indeed, if boundary condition [Eq. (68)] is used, the integration is eo:-ivalent to solving for $\phi$, and if boundary condition [Eq.*(67)] is used, the integration is equivalent to solving for $\phi^{+}$. Since $\phi$ and $\phi^{+}$are related in the contributon balance equation [Eq. (46)] by $\Omega \cdot \nabla \psi$ and since a single integration of the third-order equation will not give $\Omega \cdot \nabla \psi$, at least two integrations of Eq, (62) are required to effectively obtain both $\phi$ and $\phi^{+} . \psi$ is then obtained elther by integrating $a$ third time or by multiplying the regults for $\phi$ and $\phi "$ together according to $\mathrm{Eq}$. (5).

The following observation is made based upon Eq3. (53), (55), (69), and (70). In a $\Sigma$ region, since $S \neq 0$ and $\Delta_{2}=0$, the quantity in brackets 
In Eq. (69) must be zero if $\phi$ is to be bounded in such a region. Since $s^{+} \equiv 0$ in a $\sum$ region, the quantity in brackets reduces to the left sfde of Eq. (55), the differential equation for $\psi$ in a $\Sigma$ region. Thus, the existence of the second-order differential equation, Eq. (55), for $\psi$ in a $\sum$ region is a necessary condition for $\phi$ to be bounded in such a region. On the other hand, since $s^{+} \neq 0$ and $\Delta_{2}=0$ in a $\Sigma^{+}$region, the quantity in brackets in $\mathrm{Eq}$. (70) must be zero if $\phi^{+}$1.s to be bounded in such a region. Since $s \equiv 0$ in a $\Sigma^{+}$region, the quanticy in brackets in Eq. (70) reduces to the left side or Eq. (53), the differential equation for $\psi$ in a $\Sigma^{+}$region. Thus, the existence of the second-order differential equation, Eq. (53), for $\psi$ in a $\Sigma^{+}$reg is is a necessary condition for $\phi^{+}$ to be bounded in such a regton.

\section{Analytical Solution of Pure Absorber} Problem 1

The pure absorber problem in Fig. 4 will now be solved analytically. To sumarize, the equations for this problem are

$$
\begin{aligned}
& \mu^{2} \frac{\partial^{2} \psi(x, \mu)}{\partial x^{2}}-\sigma \mu \frac{\partial \psi(x, \mu)}{\partial x}=0,0<x<a ; \\
& \mu \frac{\partial \psi(x, \mu)}{\partial x}=0, a<x<b ;
\end{aligned}
$$

and

$$
\begin{array}{r}
\mu^{2} \frac{\partial^{2} \psi(x, \mu)}{\partial x^{2}}+\sigma_{A}^{\mu} \frac{\partial \psi(x, \mu)}{\partial x}=0, \\
b<x<c .
\end{array}
$$

The boundary conditions are

$$
\begin{aligned}
& \psi(0, \mu)=\psi(c, \mu)=0, \\
& \psi\left(a^{-}, \mu\right)=\psi\left(a^{+}, \mu\right), \\
& \psi\left(b^{-}, \mu\right)=\psi\left(b^{+}, \mu\right),
\end{aligned}
$$

The general solution to Eqs. (81)-(83) is

$$
\dot{\psi}(x, \mu)= \begin{cases}B_{1}(\mu)+A_{1}(\mu) e^{\sigma_{A} x / \mu}, & 0 \leq x \leq a \\ B_{2}(\mu), & z \leq x \leq b \\ B_{3}(\mu)+A_{3}(\mu) e^{-\sigma} x / \mu, & b \leq x \leq c .\end{cases}
$$

where $A_{1}, A_{3}, B_{1}, B_{2}$, and $B_{3}$ are arbitrary integration constants. The use of the first three bourdary conditions [Eqs. (84)-(86)] shows that

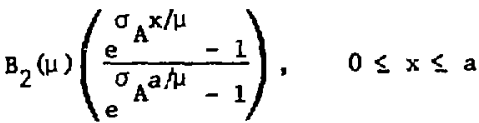

$$
\begin{aligned}
& \psi(x, \mu)= \begin{cases}B_{2}(\mu), & a \leq x \leq b \\
(-\alpha x / \mu & -\alpha c / \mu)\end{cases}
\end{aligned}
$$

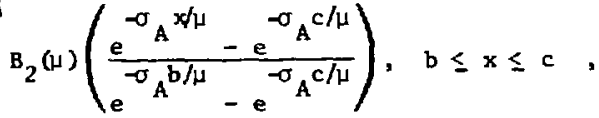

and the use of the final boundary condition [Eq. (87)], i.e., the bridging condition, gives

$B_{2}(\mu)=\left\{\begin{array}{l}0, \quad \text { or } \\ \frac{Q_{0} R_{0}}{4 \pi \sigma_{A}^{2}}\left(e^{\sigma} A^{a / 4}-1\right)\left(e^{-\sigma_{A} b / \mu}-e^{-\sigma_{A} c / \mu}\right) .\end{array}\right.$

Two solutions occur for $\mathrm{B}_{2}(\mathrm{H})$ because the nonlinearify of the bridging condition results in a second-degree algebraic equation for $B_{2}(\mu)$.

To insure proper cholce of $B_{2}(\mu)$, the contributon balance equation,

$\mu \frac{\partial \psi(x, \mu)}{\partial x}=Q(x, \mu) \phi^{+}(x, \mu)-R(x, \mu) \phi(x, \mu)$,

is evaluated at $x=0$. Inserting the solution for $\psi$ from Eq. (89) and using the vacuum boundary

and

$$
\mu^{2} \frac{\partial \psi\left(a^{-}, \mu\right)}{\partial x} \frac{\partial \psi\left(b^{+}, \mu\right)}{\partial x}+\frac{1}{4 \pi} Q_{0} R_{0} \psi(b, \mu) e^{-\sigma A^{(b-a) / \mu}}=0 \text {. }
$$


condition [Eq. (19)] for $\phi^{+}$yield

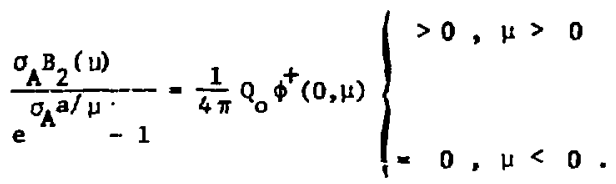

Thus,

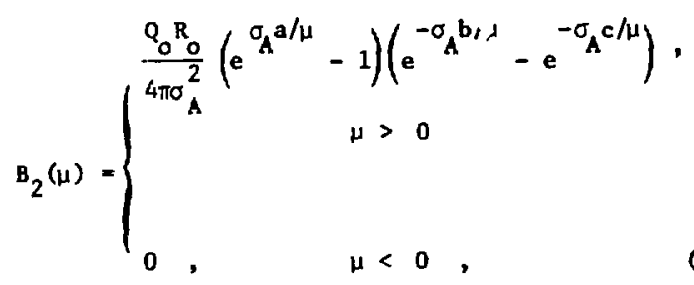

and the complete solution for this problen is

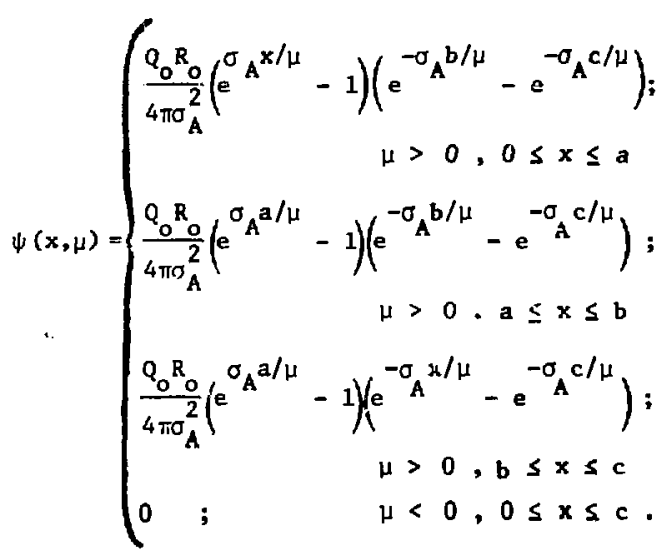

5. Numerical Solution of Pure Absorber Problem 1

This section is included to Illustrate how the nonlinearity introduced by the bridging condition [Eq. (87)] is handled in the numerical solution of the pure absorber problem. Figure 5 shows how the $\Sigma$ region $(0<x<$ a) is divided into spatial cells. The typical difference equation for this region is obtained by integrating Fq. (81), rewritten in the form

$$
\frac{\partial}{\partial x}\left[e^{-\sigma} A^{x / \mu} \frac{\partial \psi(x, \mu)}{\partial x}\right]=0
$$

from $x_{1-\frac{1}{2}}$ to $x_{1+\frac{1}{2}}$ to yleld

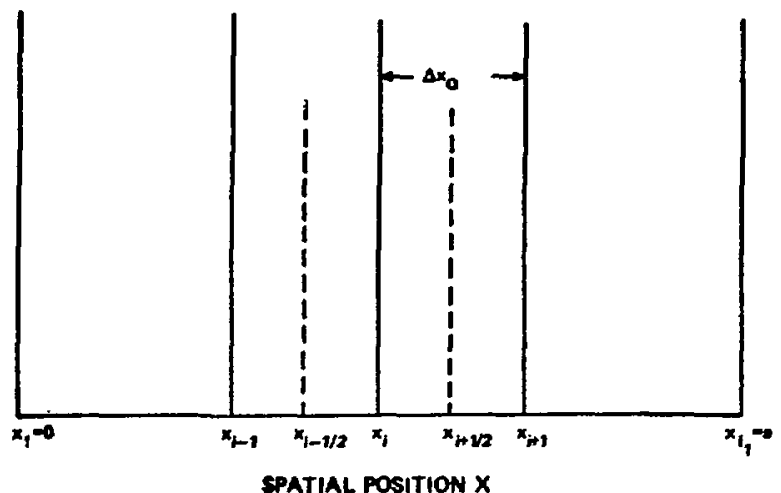

F18. 5. I region spatial cell structure for the pure absorber problen 1 .

$$
e^{-\sigma_{A} x_{1+\delta_{2}} / \mu} \frac{\partial \psi\left(x_{1+\frac{1}{2}}, \mu\right)}{\partial x}-e^{-\sigma_{A} x_{1-\frac{1}{2}} / \mu} \frac{\partial \psi\left(x_{1-\frac{1}{\Sigma}}, \mu\right)}{\partial x}=0 .
$$

Use of the central difference approximation ${ }^{12}$ for the slopes in this equation gives the typical difference equation,

$$
\begin{gathered}
\mathrm{e}^{\sigma \Delta x_{Q} / \mu} \psi_{1-1}(\mu)-\left(1+\mathrm{e}^{\sigma_{A} \Delta \mathrm{x}_{\mathrm{Q}} / \mu}\right) \psi_{1}(\mu)+\psi_{i+1}(\mu)=0 \\
1=2, \ldots, I_{1}-?
\end{gathered}
$$

Integrating Eq. (95) from $x_{I_{1}-3 / 2}$ to $x_{I_{1}}=a$ and using the central difference approximation yield

$$
\psi_{I_{1}-2}(\mu)-\psi_{I_{1}-1}(\mu)=-\Delta x_{Q} e^{-(3 / 2) J_{A} \Delta x_{Q} / \mu} \frac{\partial \psi\left(x_{I_{1}}, \mu\right)}{\partial x} \text {. }
$$

The vacuur boundary condition at $x=0$ gives the final equation for this region, namely

$$
\psi_{1}(\mu)=0
$$

The $\Sigma^{+}$region $(b<x<c)$, for which the differential equation [Eq. (83)] can be rewritten as

$$
\frac{\partial}{\partial x}\left[e^{\sigma x / \mu} \frac{\partial \psi(x, y)}{\partial x}\right]=0 \text {, }
$$

is divided into spatial cells, as shown in Fig. 6 . 
Through the use of the central difference approximation, the rypical difference equation for this

region is

$\psi_{i-1}(\mu)-\left(1+e^{\sigma_{A} \Delta x_{R} / \mu}\right) \psi_{1}(\mu)+e^{\sigma_{A} \Delta x_{R} / \mu} \psi_{1+1}(\mu)=0 ;$

$$
i=I_{2}+1, \ldots, I_{3}-1,(101)
$$

Integrating Eq. (100) from $x_{I_{2}}=b$ to $x_{I_{2}+x_{2}}$ gives

$\frac{\partial \psi\left(x_{I_{2}}^{+}, \mu\right)}{\partial x}=e^{(1 / 2) \sigma_{A} \Delta x_{R} / \mu}\left[\frac{\psi_{I_{2}+1}(\mu)-\psi_{I_{2}}(\mu)}{\Delta x_{R}}\right]$,

which is substituted Into the bridging ccndition

[Eq. (87)] to yield using the central difference approximation to give

$$
\psi_{I_{1}}(\mu)=\psi_{I_{1}-1}(\mu)+\Delta x_{Q} e^{-(1 / 2) \sigma_{A} \Delta x_{Q} / \mu \nu\left(x_{I_{1}}^{-}, \mu\right)}-\frac{1}{\partial x}
$$

Equations (97)-(99) are collected fnto one system of equations, the $\sum$ system, and Eqs. (101), (103), (104), and (106) are collected Into another system of equations, the $\Sigma^{+}$system. If the discrete-ordinates approximation ${ }^{l}$ is used, then these systems of equations are is $F \cdot$ llows:

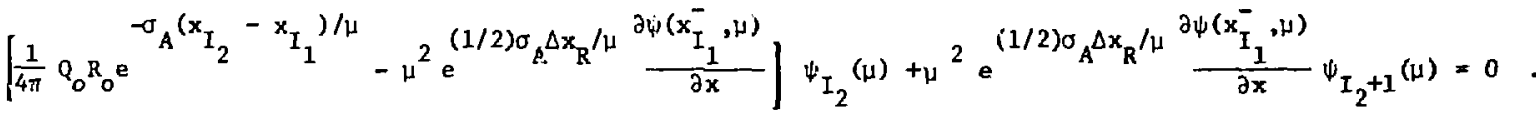

The vacuum boundary condition at $x=c$ gives the final equation for this region, namely

$$
\psi_{I_{3}}(\mu)=0
$$

If the differential equation in the 0 region $(a<x<b)$,

$$
\frac{\partial \psi(x, \mu)}{\partial x}=0 \text {, }
$$

is integrated from $x_{I_{1}}=a$ to $x_{I_{2}}=b$, the result is

$$
\dot{\psi}_{I_{1}}(\mu)-\psi_{I_{2}}(\mu)=0 ;
$$

that is, $\psi$ is spatially constant over the entire 0 region. One final equation is needed to couple the equations of the $\Sigma$ and 0 regions. This is obtained by integrating Eq. (95) from $x_{I_{1}-\frac{1}{2}}$ to $x_{I_{1}}=a$ and

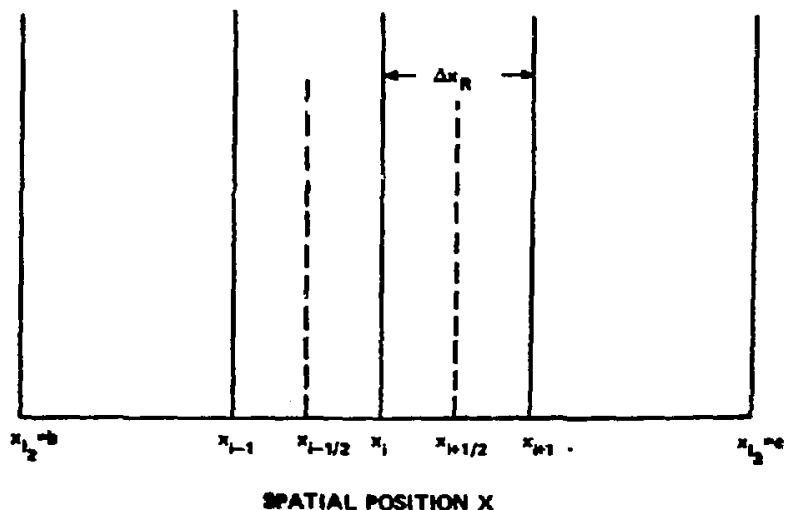

Fig. 6. $\Sigma^{+}$region spatial cell structure for the pure absorber problew 1 . 


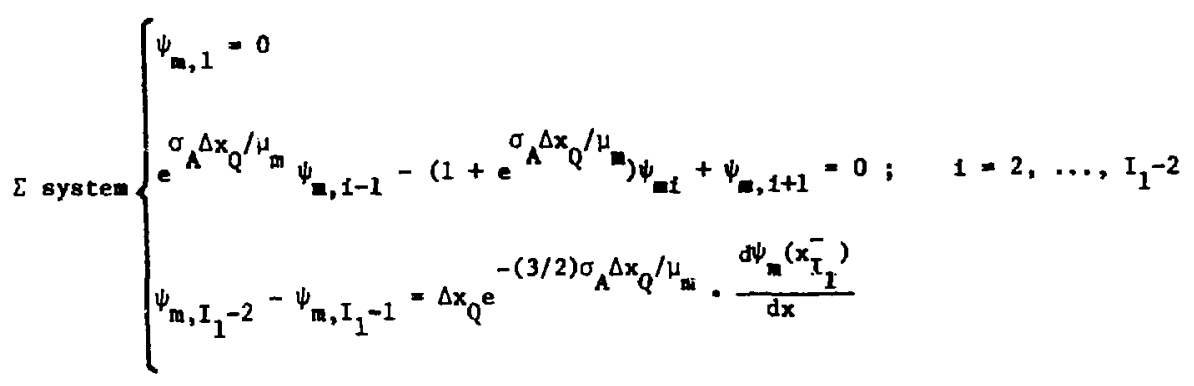

and

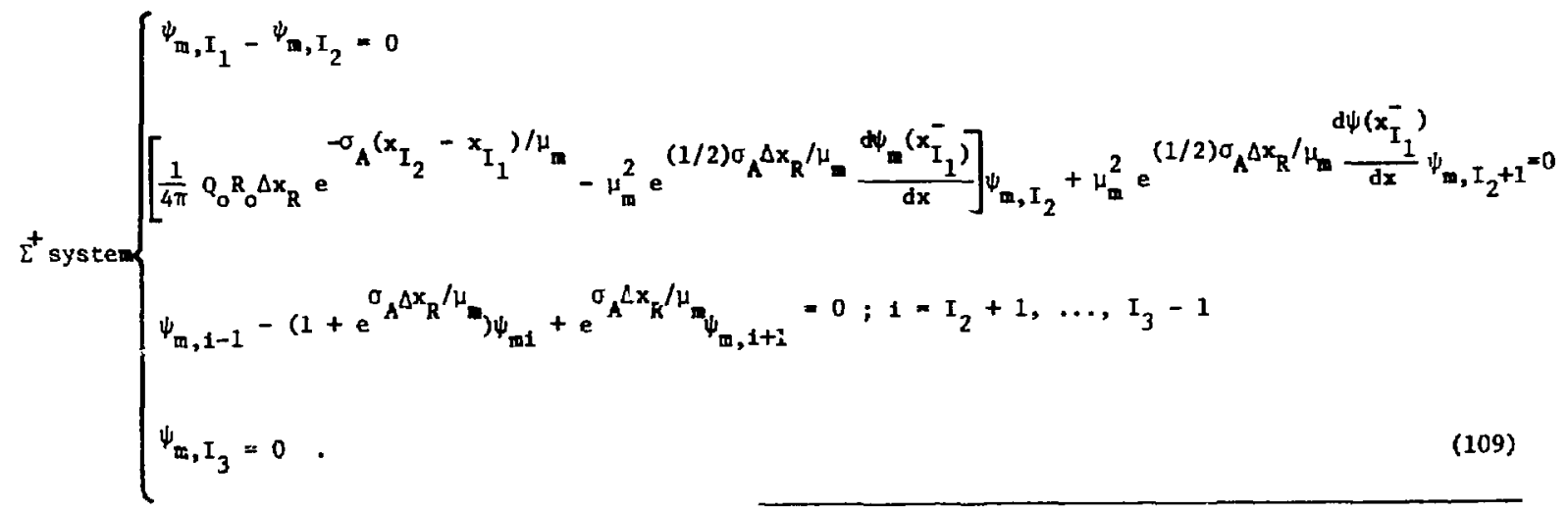

It will be noted that the $\Sigma$ systen is Inhomogeneous but that the quantity $\mathrm{d} \psi_{\mathrm{m}}\left(\mathrm{x}_{\boldsymbol{i}_{1}}^{-}\right) / \mathrm{dx}$ in the sourse vector is unknown. However, the $\Sigma^{+}$system is homogeneous, so $d \psi_{m}\left(x_{I}^{-}\right) / d x$ must have a particular value if this system is to have a nontrivial solution. Thus, $d \psi_{m}\left(x_{I_{1}}^{-}\right) / d x$ is chosen so that the determinant of the coefficlent matrix of the $\Sigma^{+}$system is zero, and with $d \psi_{m}\left(x_{I}^{-}\right) / d x$ known, the $\Sigma$ sysiem can be solved. The coupling condition [Eq. (107)] is then used to couple the $\Sigma$ and $\Sigma^{+}$systers, and the $\Sigma^{+}$system is then solved. It should be noted that this approach is not applicable for those directions $\mu_{\text {a }}$ in which $\psi_{n}(x) \equiv 0$.

The solution to this pure absorber proble for the $\mathrm{S}_{16}$ approximation is plotted in Fig. 7. The 1nportant parameters used in this calculation are $Q(x, \mu)=1 / 4 \pi$ neutrons $/ \mathrm{cm}^{3}-s \mathrm{r}-\mathrm{s}, R(x, \mu)=1$ response$\mathrm{s} / \mathrm{cm}, J_{A}=1 \mathrm{~cm}^{-1}, a=1 \mathrm{~cm}, b=9 \mathrm{~cm}$, and $c=10$ cm. The curves for $\mu<0,61787$ do not appesr since $\psi$ for those directions 18 essentially zero compared to $\psi$ for $\mu 20.61787$.

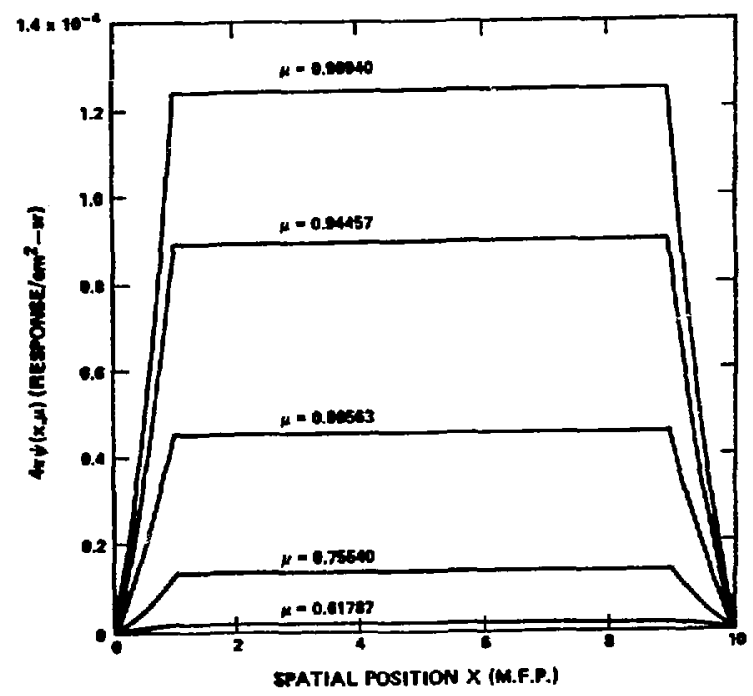

F1g. 7. Solution for pure absorber problen 1 in the $\mathrm{S}_{16}$ approximation. 


\section{Analyzical Solution of Pure Absorber Problem 2}

Consider now the pure absorber problen with $Q(x, \mu), R(x, \mu)$, and $\sigma_{A}(x)$ pasitive and uniform in $x$ in the domain $x \in(-a, a)$ and with vacuum boundarles at $x=a$ and $x=-a$. Since $Q$ and $R$ are nonzero throughout the system, the third-order equation for $\psi$ is applicable. For this problen, the third-order equation [Eq. (62)] for $\psi$ reduces to

$$
\mu^{3} \frac{\partial^{3} \psi(x, \mu)}{\partial x^{3}}-\sigma_{A}^{2} \mu \frac{\partial \psi(x, \mu)}{\partial x}=0,-a<x<a .
$$

To simplify tis solution of this prohlem, we introduce the new function,

$$
f(x, \mu)=\mu \frac{\partial \psi(x, \mu)}{\partial x}
$$

Equation (110) then becomes

$$
\mu^{2} \frac{\partial^{2} f(x, \mu)}{\partial x^{2}}-\sigma_{A}^{2} f(x, \mu)=0,-a<x<a .
$$

Equations (67) and (68) provide the following vacuum boundary conditiong for $f(x, \mu)$ :

$$
\left.\left.\begin{array}{l}
\mu \frac{\partial f(-a, \mu)}{\partial x}-\sigma_{A} f(-a, \mu)=-2 Q(\mu) R(\mu) \\
\mu \frac{\partial f(a, \mu)}{\partial x}+\sigma_{A} f(a, \mu)=-2 Q(\mu) R(\mu)
\end{array}\right\} \begin{array}{l}
\mu>0 \\
\text {. }
\end{array}\right\}
$$

and

$$
\left.\begin{array}{l}
\mu \frac{\partial f(-a, \mu)}{\partial x}+\sigma_{A} f(-a, \mu)=-2 Q(\mu) R\left(\mu^{\prime}\right) \\
\mu \frac{\partial f(a, \mu)}{\partial x}-\sigma_{A} f(a, \mu)=-2 Q(\mu) R(\mu)
\end{array}\right\} \begin{aligned}
& \mu<0 .
\end{aligned}
$$

The general solution to $\mathrm{Eq}$. (112) is just

$$
f(x, \mu)=A(\mu) e^{\sigma_{A} x / \mu}+B(\mu) e^{-\sigma_{A} x / \mu} \text {, }
$$

and the boundary conditions give the following results for the solution coefficients:

$$
A(\mu)=-\frac{Q(\mu) R(\mu)}{\sigma_{A}} e^{-\sigma_{A} a /|\mu|}
$$

and

$$
B(\mu)=\frac{Q(\mu) R(\mu)}{\sigma_{A}} e^{-\sigma_{A} a / \mu \mid} .
$$

Thus, the conplete solution for Eq. (112) with boundary conditions given by Eqs. (113) and (114) is

$$
f(x, \mu)=-\frac{2 Q(\mu) R(\mu)}{\sigma_{A}} e^{-\sigma_{A} a /|\mu|} \sinh \frac{\sigma_{A} x}{\mu} .
$$

Through the use of Eqs. (69), (70), (111), and (118), the quantities $\phi$ and $\phi^{+}$are found to be

$$
\phi(x, \mu)=\frac{Q(\mu)}{\sigma_{A}}\left[1-e^{-\sigma_{A}\left(\frac{a}{|\mu|}+\frac{x}{\mu}\right)}\right]
$$

and

$$
\phi^{+}(x, \mu)=\frac{R(\mu)}{\sigma_{A}}\left[1-e^{-\sigma_{A}\left(\frac{a}{|\mu|}-\frac{x}{\mu}\right)}\right] .
$$

Thus, solving Eq. (112) with the boundary conditions given by Eqs. (113) and (114) is equivalent to solving for $\phi$ and $\phi^{+}$. At this point $\psi(x, \mu)$ can be obtained either by multiplying these results for $\phi$ and $\phi^{+}$together or by solving equation (111) with the boundary conditions,

$$
\psi(-a, \mu)=0, \mu>0
$$

and

$$
\psi(a, \mu)=0, \mu<0 .
$$

In elther case, the result is

$$
\psi(x, \mu)=\frac{2 \Omega(\mu) R(\mu)}{\sigma_{A}^{2}} e^{-\sigma_{A} a^{a}|\mu|}\left(\cosh \frac{\sigma_{A}^{a}}{\mu}-\cosh \frac{\sigma_{A}^{x}}{\mu}\right) .
$$


lure absorber problen 1 can also be nolved by wans of the third-order equation for $\psi$. The aseoclated problen with

$Q(x, \mu)= \begin{cases}\frac{Q_{0}}{4 \pi}>0, & 0<x<a \\ \frac{Q_{1}}{4 \pi}>0, & ,<<c\end{cases}$

and

$R(x, \mu)=\left\{\begin{array}{rr}R_{1}>0, & 0<x<b \\ R_{0}>0, & b<x<c\end{array}\right.$

1s first solved. The approprlate differential equation 18

$$
\mu^{2} \frac{\partial^{2} f(x, \mu)}{\partial x^{2}}-\sigma_{A}^{2} f(x, \mu)=0 \text {. }
$$

and the boundary condittons at che externel vacuu boundarles are

$$
\left.\begin{array}{l}
\mu \frac{\partial f(0, \mu)}{\partial x}-\sigma_{A} f(0, \mu)=-\frac{1}{2 \pi} Q_{0} R_{1} \\
\mu \frac{\partial f(c, \mu)}{\partial x}+\sigma_{A} f(c, \mu)=-\frac{1}{2 \pi} Q_{1} \mu_{0}
\end{array}\right\} \mu>0 \text { (125) }
$$

and

$$
\left.\begin{array}{l}
\mu \frac{\partial f(0, \mu)}{\partial x}+\sigma_{A} f(0, \mu)=-\frac{1}{2 \pi} Q_{0} R_{1} \\
\mu<0 .(126) \\
\mu \frac{\partial f(c, \mu)}{\partial x}-\sigma_{A} f(c, \mu)=-\frac{1}{2 \pi} Q_{1} R_{0}
\end{array}\right)
$$

The boundary conditione the interfaces, $x=a$ and $x=b$, are obtained by requiring continulty of and $\phi^{+}$and are

$$
\begin{aligned}
& \mu \frac{\partial f\left(a^{-}, \mu\right)}{\partial x}-\sigma_{A} f\left(a^{-}, \mu\right)+\frac{1}{2 \pi} Q_{0} R_{1} \\
& -\mu \frac{\partial f\left(a^{+}, \mu\right)}{\partial x}-\sigma_{A} f\left(a^{+}, \mu\right)+\frac{1}{2 \pi} Q_{1} R_{1}, \\
& \frac{\partial f\left(a^{-}, \mu\right)}{\partial x}+\sigma_{A} E\left(a^{-}, \mu\right)+\frac{1}{2 \pi} Q_{0} K_{1}
\end{aligned}
$$

$$
=\frac{Q_{0}}{Q_{1}}\left[\mu \frac{\partial f\left(a^{+}, \mu\right)}{2 x}+o_{a} f\left(a^{+}, \mu\right)+\frac{1}{2 \pi} Q_{1} R_{1} l,\right.
$$

$$
\begin{aligned}
& , \frac{\partial f\left(b^{-}, \mu\right)}{\partial x}-\sigma_{A} f\left(b^{-}, \mu\right)+\frac{1}{2 \pi} Q_{1}{ }_{1} \\
& \quad=\frac{R_{1}}{R_{0}}\left[\mu \frac{\partial E\left(b^{+}, \mu\right)}{\partial x}-\sigma_{A} f\left(b^{+}, \mu\right)+\frac{1}{2 \pi} Q_{1} R_{0}\right] .
\end{aligned}
$$

and

$$
\begin{aligned}
& \mu \frac{\partial f\left(b^{-}, \mu\right)}{\partial x}+\sigma_{A} f\left(b^{-}, \mu\right)+\frac{1}{2 \pi} Q_{1} R_{1} \\
& \quad=\mu \frac{\partial f\left(b^{+}, \mu\right)}{\partial x}+\sigma_{A} f\left(b^{+}, \mu\right)+\frac{1}{2 \pi} Q_{1} R_{0} .
\end{aligned}
$$

Once the solution $f(x, \mu)$ to this assoctated problem 1s obtalned, the linft is taken as $Q_{1}+0$ and $R_{1} \rightarrow 0$. The result is Identical to the result obtained in Sec. IV.A.4 of this report.

\section{Numerical Solution of the Third-Order Con-} tributon Transport Equation

To study posatble implications assoclated with the numerical solution of the third-order differential equation for $\psi(x, \mu)$ for a wore general class of problews, we implement in this section a numerical algorithm to solve Eq. (62) In slab geowetry using a diecrete-ordinates approach. With the definition

$$
f(x, \mu)=\mu \frac{\partial \psi(x, \mu)}{\partial x}
$$

the third-order equation in slab geometry can be yriteten 
$\mu^{2} \frac{\partial}{\partial x}\left[A(x, \mu) \frac{\partial E(x, \mu)}{\partial x}\right]+B(x, \mu) f(x, \mu)=D(x, \mu)$.

(132)

where

$$
\begin{aligned}
A(x, \mu) & =\frac{1}{\Delta_{2}(x, \mu)} \\
& =\frac{2}{b(x, \mu)\{a(x, \mu)+c(x, \mu)\}} .
\end{aligned}
$$

$$
D(x, \mu)=\frac{\mu}{[b(x, \mu)]^{1 / 2}} \frac{\partial}{\partial x}\left\{A(x, \mu)[b(x, \mu)]^{3 / 2}\right\},
$$

$a(x, \mu)=\frac{1}{S(x, \mu)}\left[\mu \frac{\partial S(x, \mu)}{\partial x}+\sigma(x) S(x, \mu)\right]$,

$b(x, \mu)=-2 s(x, \mu) s^{+}(x, \mu)$,

and

$$
c(x, \mu)=\frac{1}{s^{+}(x, \mu)}\left[-\mu \frac{\partial s^{+}(x, \mu)}{\partial x}+\sigma(x) s^{+}(x, \mu)\right\}
$$

Fron Eqs. (67) and (68), the boundary conditions on $f(x, \mu)$ at the vacuum boundaries, $x=0$ and $x=\ell>0$, are obtained:

$\mu \frac{\hat{o} f(0, \mu)}{\partial x}-a(0, \mu) f(0, \mu)=b(0, \mu), \mu>0 ;$

$u \frac{\partial f(0, \mu)}{\partial x}+c(0, u) f(0, \mu)=b(0, \mu), \mu<0 ;$

$\mu \frac{\partial E(\ell, \mu)}{\partial x}+c(\ell, \mu) f(\ell, \mu)=b(\ell, \mu), \mu>0$;

and
At an internal boundary, $x=\tau$, continuity of $\phi$ and $\phi^{+}$gives the conditions,

$A\left(t^{-}, \mu\right) S\left(t^{-}, \mu\right)\left[\mu \frac{\partial f\left(t^{-}, \mu\right)}{\partial x}-a\left(t^{-}, \mu\right) f\left(t^{-}, \mu\right)-b\left(t^{-}, \mu\right)\right]$ 
and

(3) $D(x, \mu) \approx D_{1}(\mu)$ in the interval $\left(x_{1-\frac{1}{2}}, x_{1+\frac{1}{1}}\right)$,

(4) the central difference approxteation for $\frac{\partial f}{\partial x}$ at $x_{1 \pm \frac{1}{2}}$.

Equation (145) holds if $x_{1}$ is not the location of an external boundary or a epatial discontinuity in $S$ or $\mathrm{s}^{+}$(for example, at a material interface), If $x_{1}$ is an external boundary, then Eq. (132) is inregrated over the half-cell adjacent to the boundary. For example, at the left-hand vacuum boundary $x_{1}=0$, this integration gives

$$
\left[B_{1}(\mu) \frac{\Delta x^{2}}{2}+k_{3 / 2}(\mu)\right] f_{1}(\mu)-k_{3 / 2}(\mu) E_{2}(\mu)=D_{1}(\mu) \frac{\Delta x^{2}}{2}-k_{1}(\mu) \Delta x \frac{\partial E(0, \mu)}{\partial x} .
$$

If Eqs. (139) and (140) are solved for $\frac{\partial f(0, \mu)}{\partial x}$ and the results are used in Eq. (146), the resuit is

$$
\left.i B_{1}(\mu) \frac{\Delta x^{2}}{2}+k_{3 / 2}(\mu)+\frac{a_{1}(\mu) \Delta x}{\mu} k_{1}(\mu)\right] f_{1}(\mu)-k_{3 / 2}(\mu) f_{2}(\mu)=D_{1}(\mu) \frac{\Delta x^{2}}{2}-\frac{b_{1}(\mu) \Delta x}{\mu} k_{1}(\mu), \mu>0
$$

and

$$
\left[B_{1}(\mu) \frac{\Delta x^{2}}{2}+k_{3 / 2}(\mu)-\frac{c_{1}(\mu) \Delta x}{\mu} k_{1}(\mu)\right] f_{1}(\mu)-k_{3 / 2}(\mu) f_{2}(\mu)=D_{1}(\mu) \frac{\Delta x^{2}}{2}-\frac{b_{1}(\mu) \Delta x}{\mu} k_{1}(\mu), \mu<0 .
$$

Similarly, at the right-hand vacuum boundary $x_{I}=\ell$, Integration of Eq. (132) and use of Eqs. (141) and (142) yield

$$
\left[B_{I}(\mu) \frac{\Delta x^{2}}{2}+k_{I-b_{2}}(\mu)+\frac{c_{I}(\mu) \Delta x}{\mu} k_{I}(\mu)\right] f_{I}(\mu)-k_{I-b_{I}}(\mu) f_{I-1}(\mu)=D_{I}(\mu) \frac{\Delta x^{2}}{2}+\frac{{ }^{b}{ }_{I}(\mu) \Delta x}{\mu} k_{I}(\mu), \mu>0,
$$

and

$$
\left[B_{I}(\mu) \frac{\Delta x^{2}}{2}+k_{I-b_{2}}(\mu)-\frac{a_{I}(\mu) \Delta x}{\mu} k_{I}(\mu)\right] f_{I}(\mu)-k_{I-1}(\mu) f_{I-I}(\mu)=D_{I}(\mu) \frac{\Delta x^{2}}{2}+\frac{b_{I}(\mu) \Delta x}{\mu} k_{I}(\mu), \mu<0 .
$$

The sftuation at an interface, $x=t$, where $s$ or $s^{+}$is spatially discontinuous, is somewhat wore complicated since $f$ can be spatielly discontinuous. Integration of Eq. (132) over the half-cell to the left of the interface gives

$$
\left[B_{L}(\mu) \frac{\Delta x^{2}}{2}+k_{L-\frac{1}{2}}(\mu)\right] f_{L}(\mu)-k_{L-\frac{1}{2}}(\mu) f_{L-L}(\mu)=D_{L}(\mu) \frac{\Delta x_{L}^{2}}{2}+k_{L}(\mu) \Delta x_{L} \frac{\partial f\left(c^{-}, \mu\right)}{\partial x},
$$




$$
\left[B_{R}(\mu) \frac{\Delta x_{R}^{2}}{2}+k_{R+\zeta_{2}}(\mu)\right] f_{R}(\mu)-k_{R+\frac{l}{2}}(\mu) f_{R+1}(\mu)=D_{R}(\mu) \frac{\Delta x_{R}^{2}}{2}-k_{R}(\mu) \Delta x_{R} \frac{\partial f\left(t^{+}, \mu\right)}{\partial x},
$$

where the subscript $R$ indicates that quantities are to be evaluated at $x=t^{+}$.

The determinant $\Delta_{1}(\mu)$ of the coefficient matrix of the vector,

$$
\left[\begin{array}{l}
\mu \frac{\partial f\left(t^{-}, \mu\right)}{\partial x} \\
\mu \frac{\partial f\left(t^{+}, \mu\right)}{\partial x}
\end{array}\right] \text {, }
$$

for the system corsisting of Eqs. (143) and (144) is

$$
\Delta_{1}(\mu)=s_{R}(\mu) s_{L}^{+}(\mu)-s_{L}(\mu) s_{R}^{+}(\mu),
$$

where the subscripts, $R$ and $L$, have the interpretation just described. If $\Delta_{1}(\mu) \neq 0$, then Eqs. (143) and (144) can be solved for $\frac{\partial f\left(t^{-}, \mu\right)}{\partial x}$ and $\frac{\partial f\left(t^{+}, \mu\right)}{\partial x}$ and the results can then be substituted into Eqs.
A cuick look at Eqs. (145), (147)-(150), (154), and (155) shows that the coefficier.t matrix for the system of equations is tridiagonal. However, the coefficlent matrix will be symetric orly if $\Delta x_{L}=\Delta x_{K}$ at each internal boundary.

If $\Delta_{1}(\mu)$ of $\mathrm{Eq}$. (153) vanishes, then it is iapossible to solve Eqs. (143) and (144) for the derivatives of $f$ at the interface, and another approach must be employed. If the contributon balance equation [Eq. (46)] is rewritten in terms of $f(x, \mu)$ on the two sides of the interface at $x=t$, then we obtain the equations.

$$
f\left(t^{-}, \mu\right)=S\left(t^{-}, \mu\right) \phi^{+}(t, \mu)-s^{+}\left(t^{-}, \mu\right) \phi(t, \mu)(157 a)
$$

and

$$
f\left(t^{+}, \mu\right)=s\left(t^{+}, \mu\right) \phi^{+}(t, \mu)-s^{+}\left(t^{+}, \mu\right) \phi(t, \mu) \cdot(157 b)
$$

(151) and (152) to yield

$$
-k_{L-\frac{1}{2}}(\mu) f_{L-1}(\mu)+\left[B_{L}(\mu) \frac{\Delta x_{L}^{2}}{2}+k_{L-\frac{1}{2}}(\mu)+\frac{k_{L}(\mu) \Delta x_{L}}{\mu} F_{L}(\mu)\right] f_{L}(\mu)-\frac{\mu \Delta x_{L}}{\Delta_{1}(\mu)} f_{R}(\mu)=D_{L}(\mu) \frac{\Delta x_{L}^{2}}{2}+\frac{k_{L}(j) \Delta x_{L}}{\mu} b_{L}(\mu)
$$

and

$$
-\frac{\mu \Delta x_{R}}{\Delta_{1}(\mu)} f_{L}(\mu)+\left[B_{R}(\mu) \frac{\Delta x_{R}^{2}}{2}+k_{R+\frac{1}{2}}(\mu)+\frac{k_{R}(\mu) \Delta x_{R}}{\mu} F_{R}(\mu)\right] f_{R}(\mu)-k_{R+\frac{1}{2}}(\mu) f_{R+1}(\mu)=D_{R}(\mu) \frac{\Delta x_{R}^{2}}{2}-\frac{k_{R}(\mu) \Delta x_{R}}{\mu} b_{R}(\mu),
$$

where

$$
F_{L}(\mu)=\frac{s_{L}(\mu) s_{R}^{+}(\mu) a_{L}(\mu)+s_{R}(\mu) s_{L}^{+}(\mu) c_{L}(\mu)}{\Delta_{1}(\mu)}
$$

and

$$
E_{R}(\mu)=\frac{s_{R}(\mu) s_{L}^{+}(\mu) a_{R}(\mu)+s_{L}(\mu) s_{R}^{+}(\mu) c_{R}(\mu)}{\Delta_{1}(\mu)}
$$

If Eq. (157a) is multiplied by $s\left(t^{+}, \mu\right)$ and Eq. (157b) is multiplied by $S\left(t^{-}, \mu\right)$ and the second result is subtracted from the first, then we obtain

$$
f_{R}(\mu)=\frac{s_{R}(\mu)}{s_{L}(\mu)} f_{L}(\mu),
$$

where the subscripts, $L$ and $R$, again iave the previously described interpretation. If $\mathrm{Eq}$. (158) and Eq. (153) with $\Delta_{1}(\mu)=0$ are used in Eq. (143), then a second condition at the interface is 


$$
\mu \frac{\partial f\left(t^{+}, \mu\right)}{\partial x}=b_{R}(\mu)+\frac{A_{L}(\mu) S_{L}(\mu)}{A_{R}(\mu) S_{R}(\mu)}\left[\mu \frac{\partial f\left(t^{-}, \mu\right)}{\partial x}-b_{L}(\mu)\right]+\left[\frac{a_{R}(\mu) c_{L}(\mu)-a_{L}(\mu) c_{R}(\mu)}{a_{L}(\mu)+c_{L}(\mu)}\right] \frac{S_{R}(\mu)}{S_{L}(\mu)} f_{L}(\mu) .
$$

If Eqs. (158) and (157) are then used in Eqs. (151)

and (152), the results are

$$
\left[B_{L}(\mu) \frac{\Delta x_{L}^{2}}{2}+k_{L-\frac{1}{2}}(\mu)\right] f_{L}(\mu)-k_{L-\frac{1}{2}}(\mu) f_{L-1}(\mu)-k_{L}(\mu) \Delta x_{L} \frac{\partial f\left(t^{-}, \mu\right)}{\partial x}=D_{L}(\mu) \frac{\Delta x_{L}^{2}}{2}
$$

and

$$
\begin{aligned}
& {\left[B_{R}(\mu) \frac{\Delta x_{R}^{2}}{2}+k_{R+\frac{s_{2}}{2}}(\mu)+\frac{a_{R}(\mu) c_{L}(\mu)-a_{L}(\mu) c_{R}(\mu)}{a_{L}(\mu)+c_{L}(\mu)} \frac{k_{R}(\mu) \Delta x_{R}}{\mu}\right] \frac{s_{R}(\mu)}{s_{L}(\mu)} f_{L}(\mu)-k_{R+\frac{1}{2}}(\mu) f_{R+1}(\mu)} \\
& \quad+k_{L}(\mu) \Delta x_{R} \frac{s_{L}(\mu)}{s_{R}(\mu)} \frac{\partial f\left(t^{-},:\right)}{\partial x}=D_{R}(\mu) \frac{\Delta x_{R}^{2}}{2}-\frac{k_{R}(\mu) \Delta x_{R}}{\mu} b_{R}(\mu)+\frac{k_{L}(\mu) \Delta x_{R} s_{L}(\mu)}{\mu} \frac{s_{R}(\mu)}{s_{L}}(\mu) .
\end{aligned}
$$

Examination of Eqs. (145), (147)-(150), (160), and (161) a a complete set of difference equations shows that the coefficlent matrix is no longer tridiagonal when $\Delta_{1}(\mu)=0$.

The set of difference equations consisting of Eqs. (145), (147)-(150), (154), and (155) has been employed to solve some simple problems in slab geometry using the discrete-ordinates approach. The first set of problems consisted of the two pure absorber problems described in Sec. IV.A.6:

(1) pure absorber problem 2, in which the source distribution $Q$, detector distribution $R$, and the absorption cross section were spatially uniform, and

(2) the modified pure absorber problem 1 , in which the source and detector distributions were discontinuous, at described by Eqs. (122) and (123), and the thickness $(b-a)$ of the central region was set to zero.

The numerical results indicate that, when spatial discontinuities exist in $Q$ or $R$, these difference equations are susceptible to numerfcal instability. This Instability is manifested in the fact that the contributon flux solution may be extremely sensitive to the choice of the size of the spatial mesh interval $\Delta x$ for a spectelc range of $\Delta x$ values. It is shown in Appendix $A$ for a simple case that when $Q$ and $R$ satisfy certain conditions, there is at least one critical value, $\Delta x_{c}>0$, for which the determinant of the coefficient matrix of the system of difference equations vanishes. The solution $f(x, \mu)$, and consequently $\psi(x, \mu)$, will be extremely sensitive to the choice $\Delta \mathrm{x}$, when $\Delta \mathrm{x}$ is near this critical value, $\Delta \mathrm{x}_{\mathrm{c}}{ }^{*}$ When source discontinuities were nonexistent, no such problem with numerical instability was observed.

The second set of problems consisted of problems with scattering. The coefficients in the difference equations for these problems were calculated from source distributions, $S$ and $\mathrm{s}^{+}$, which were generated from forward and adjoint fluxes computed with the discrete-ordinates code, ONETRAN, for the finest mesh studied. The distributions, $s$ and $s^{+}$, for coarser meshes were also obtained from these fine-mesh data, through interpolation where necessary. For the purpose of these calculations, a spectal $O^{\prime}$ region is defined to be a scattering region in which both $Q$ and $R$ are identically zero. The geometry for the typical scattering problem is shown in Fig. 8 for the deepest penetration studied, namely 50 mean-free paths in the central $0^{\prime}$ region. The corresponding problem for a penetration of 20 mean-free paths was also investigated. In both sets of calculations, a variety of scattering-to-cotal ratios $\left(\sigma_{\text {so }} / \sigma=0.2\right.$, 0.6 , and 1.0 ) was employed, and the results of these calculations are summarized in Tables II and III, where the integral response $I$ has been computed from Eq. (5c). The results for problems with some absorption Indicate that the difference equations may be susceptible to the same type of numerical instability 


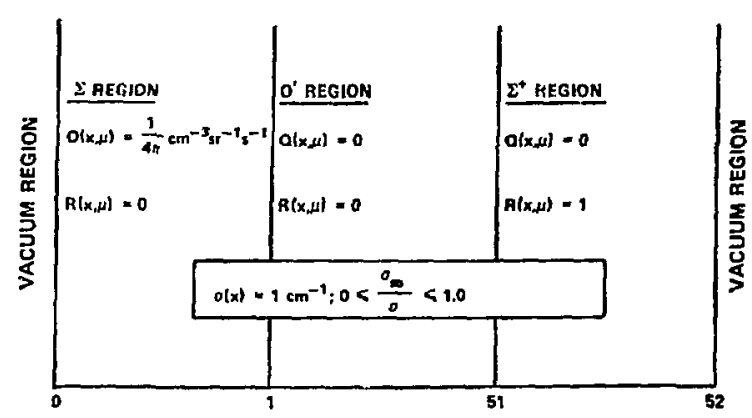

SPATIAL POSITION, NOT DRAWN TO SCALE (M.F.P.)

INTEGRAL RESPONSE $I=\frac{2 \pi}{50} \int_{1}^{51} \int_{-1}^{1} \mu \nu\left(x_{n} \mu\right) d \mu d x$

Fig. 8. Geometry for deep-penetration problem.

observed in the pure absorber problems. However, for extremely coarse spatial meshes, the in agral response is seen to be relatively insensitive to the mesh size for the particular values of $\Delta x$ used. This is not to say that regions of Instability do not exist for coarse spatial meshes, because theee regions of instability may have been missed by fortuitous choice of $\Delta x$ values. On the other hand, the results for the pure scattering problem $\left(\sigma_{\text {so }} / \sigma=\right.$ 1.0) do not indicate any instablitty, but they do show the effect of using too coarse a mesh for problems with considerable spatial curvature in $f(x, \mu)$.

The difficulty encountered in solving the contributon difference equations for problems with source discontinulties is not totally unexpected, since the unknown $f(x, y)$ is discontinuous at the location of discontinuities in $s(x, \mu)$ and $s^{+}(x, \mu) .^{13}$ In the set of difference equations, it is the equations for the cells in the immediate vicinity of source discontinuities that show the greatest tendency toward causing loss of diagonal dominance in the coefficient matrix of the system of difference

TABLE II

SENSITIVITY OF THE INTEGRAL RESPONSE I FROM THE QUASI-LINEAR CONTIIBUTCN APPROACH TO THE MESH SIZE IN THE CENTRAI O' REGION

(50 MEAN-FREE PATHS PENETRATION)

\begin{tabular}{l}
$\begin{array}{c}0 \text { Region } \\
\sigma \Delta \mathrm{x} \\
\text { (m.f.p) }\end{array}$ \\
\hline $\begin{array}{l}\text { Reference } \\
0.390625\end{array}$ \\
0.78125 \\
1.5625 \\
3.125 \\
6.25 \\
12.5 \\
25
\end{tabular}

a Integral response I calculated from Eq. (5c).

b Reference response using the iterattve contributon approach described in Sec. IV.C with $\sigma \Delta x=$ $0.05 \mathrm{In} 0^{\prime}$ Iegion. The corresponding responses using ONETRAN forward and adjoint fluxes computed with $\sigma \Delta x=0.1 \subseteq j 3125$ in the $0^{\prime}$ region are $5.73 \times 10^{-26}$ for $\sigma_{\text {so }} / \sigma=0.2$ and $2.22 \times 10^{-21}$ for $\sigma_{\mathrm{so}} / \sigma=0.6$. These ONETRAN results probably exhibit a larger remaining error than the iterative contributon results quoted in the Table because a much coarser spatial mesh had to be used.

${ }^{C}$ Reference response using ONETRAN forward and adjoint fluxes computed with $\sigma \Delta x=0.390625$ in the
$0^{\prime}$ region.

$$
\begin{aligned}
& \frac{\sigma_{\text {so }}}{\sigma}=1.0 \\
& 7.34 \times 10^{-2 c} \\
& 7.59 \times 10^{-2} \\
& 7.79 \times 10^{-2} \\
& 8.09 \times 10^{-2} \\
& 7.94 \times 10^{-2} \\
& 5.73 \times 10^{-2} \\
& -1.76 \times 10^{-2} \\
& -2.91 \times 10^{-1}
\end{aligned}
$$


(1) lack of ronsistency between the contributon difference equations and the forward and adjoint difference equations, in the sense described in Sec. IV.C.4,

(2) errors introduced into $\phi$ and $\phi^{+}$by replauing the derivatives in Eqs. (69) and (70) by numerical approximutions, and

(3) an interaction between the previously described numertcal instability and changes in the shapes of $S$ and $S^{+}$in successive iterations.

It is felt that the first two, which may not be totally unrelated to each other, are the most probable of the three possibilities. The third possibility, although remote, is an interesting one, but it is not an obvious one since $\Delta x$ is not varied in successive iterations. What can happen, however, is that the values $\Delta x_{c}$, for which the determinant of the coefficlent matrix vanishes, will vary from iteration to iteration. This occurs since the elements of the coefficient matrix are computed from the distributions, $s^{(k-1)}$ and $s^{+(k-1)}$, and these distributions vary with iteration. Thus, in successive iterations, the values of $\Delta x_{c}$ can move toward or away from the actual value $\Delta x$ being used for the calculation. If such shifting in the values of $\Delta x_{c}$ should place $\Delta x$ in a region of large solution sensitivity, the result could be an interference with the iterative convergence.

B. A Nonlinear Contributon Transport Equation

A generalization of Eq. (23) in Ref. 2 is easily obtained for a $\Sigma \Sigma^{+}$region $\left(s, s^{+} \neq 0\right.$ ) by multiplying the expressions (69) and (70) for $\phi$ and $\phi^{+}$together to give the second-order nonlinear differential equation,

$$
\begin{aligned}
& \left.\left[(\underline{\Omega} \cdot \nabla)^{2} \psi\right]^{2}+4 S S^{+}(\underline{\Omega} \cdot \nabla)^{2} \psi-\frac{1}{2}\left(\frac{1}{S^{+}} \underline{\Omega} \cdot \nabla S^{+}+\frac{1}{S} \underline{\Omega} \cdot \nabla S\right) \underline{\Omega} \cdot \nabla[\underline{(\Omega \cdot \nabla \psi})^{2}\right]-\left(\sigma-\frac{1}{S} \underline{\Omega} \cdot \nabla S^{+}\right)\left(\sigma+\frac{1}{S} \underline{\Omega} \cdot \nabla S\right)(\underline{\Omega} \cdot \nabla \psi)^{2} \\
& -2\left(\mathrm{~S} \underline{\Omega} \cdot \nabla \mathrm{S}^{+}+\mathrm{s}^{+} \underline{\Omega} \cdot \nabla \mathrm{s}\right) \underline{\Omega} \cdot \nabla \psi-\frac{\left(\Delta_{2}\right)^{2}}{\mathrm{SS}^{+}} \psi+4\left(\mathrm{SS}^{+}\right)^{2}=0 ; \quad(\underline{\mathrm{r}}, \underline{\Omega}, \mathrm{E}) \varepsilon \Sigma \Sigma^{+} .
\end{aligned}
$$

For $\Sigma, \Sigma^{+}$, and 0 regions, the linear differential equations [Eqs. (47), (53), and (55)] are still approprlate. For the case of a pure absorber with uniform external sources, Eq. (162) reduces in slab geometry to Eq. (23) of Ref. 2.

The external vacuum boundary condition for Eq. (162) is

$$
\left.\psi\left(\underline{\varepsilon_{b}}, \underline{\Omega}, E\right)=0 \text { for all } \underline{\underline{\Omega}}, \mathrm{E}\right)
$$

on the boundary surface. Possible boundary conditions at a material interface are

$$
\begin{aligned}
& \psi(\underline{I}, \underline{\Omega}, E) \text { is continuous in } E \text {, } \\
& \frac{S}{\Delta_{2}}\left[(\underline{\Omega} \cdot \nabla)^{2} \psi-\left(\sigma+\frac{1}{S} \underline{\Omega} \cdot \nabla s\right) \underline{\Omega} \cdot \nabla \psi+2 s S^{+}\right] \text {is } \\
& \frac{s^{+}}{\Delta_{2}}\left[(\underline{\Omega} \cdot \nabla)^{2} \psi+\left(\sigma-\frac{1}{s^{+}} \Omega \cdot \nabla s^{+}\right) \Omega \cdot \nabla \psi+2 s s^{+}\right] \text {is } \\
& \text { continuous in } \underline{r} \text {. }
\end{aligned}
$$

According to Eqs. (69) and (70) these correspond to continuity in $\psi, \phi$, and $\phi^{+}$, respectively, so at most two will be independent when used with Eq. (162). Because of the obvious difficulty involved in solving Eq. (162), this approach was pursued no further at this time.

c. An Iterative Solution to the Contributon Transport Problem

This approach involves transforming the forward and adjoint Boltzmann squations in such a way that the terms which appear in the new equalions are slowly varying in the spatial variable over much of the system. The hope is that a coarser mesh can be used for the numerical solution of such equations than could have been used for the original equations. 1. Derivation of the Transformed Equaticns We begin with the forward Boltzmann equation

$$
\underline{\Omega} \cdot \nabla \phi+\sigma \phi=\mathrm{S} .
$$

The quantity $\phi$ is replaced by the product $f \psi$, where $\psi$ will become the primary unknown and will depend upon the, as yet, unspectified function $f$. If this replacement is made, Eq. (164) becomes

$$
\Omega \cdot \nabla \psi+\left(\sigma+\frac{1}{f} \underline{\Omega} \cdot \nabla f\right) \psi=\frac{S}{E} .
$$


Ir the contributon transport problem, we iden$t+5 y \forall$ as the contributon flux, so that

$$
\mathbf{f} \equiv \frac{1}{\phi^{+}},
$$

and $\mathrm{Eq} .(165)$ becomes

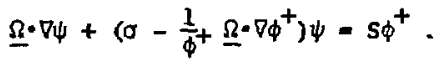

Since Ey. (167) is obtained from the forward Boltznann equation [Eq. (164)] by replacing $\phi$ with $\psi / \phi^{+}$, the function $\psi / \phi^{+}$satisfies the forward Boltzmann equation. However, the identity,

$$
\frac{\psi}{\phi^{+}} \equiv \phi,
$$

where $\phi$ is the forward flux, holds only if $\frac{\psi}{\phi^{+}}$satisfies the boundary conditions appropriate for the forward flux $\phi$. Since $\phi^{+}$is the adjoint flux, this means that in solving Eq. (167) for $\psi$ we must require $\psi$ to satisfy the same boundary conditions as $\phi$ (for example, $\psi=0$ for incoming directions at a vacuum boundary). We note, however, that the complete set of boundary conditions for $\psi$ can be determined from the independent boundary conditions on $\phi$ and $\phi^{+}$, as discussed in Sec. II.B. This means that we have used only half the contributon flux boundary conditions in solving Eq. (167) for $\psi$, namely those obtained from the boundary conditions for $\phi$. The fact that $\psi$ also satisfies the complete set of contributon flux boundary conditions and is the contributon flux, $\psi=\phi \phi^{+}$, is guaranteed by the relationship in Eq. (168) only because $\phi^{+}$is the adfolnt flux.

The corresponding transformation of the adjoitic Boltzmann equation [Eq. (45)] In the contributon approach is made by replacing $\phi^{+}$with $\bar{\psi} / \phi$ to give

$$
-\underline{\Omega} \cdot \nabla \bar{\psi}+\left(\sigma+\frac{1}{\phi} \underline{\Omega} \cdot \nabla \phi\right) \bar{\psi}=s^{+} \phi .
$$

Thus, the function $\frac{\bar{\Psi}}{\phi}$ satisfies the adjoint Boltzmann equation, but the identity,

$$
\bar{\psi} \equiv \phi^{+}
$$

where $\phi^{+}$is the adjoint flux, holds only if $\frac{\bar{\psi}}{\phi}$ satisfles the boundary conditions appropriate for the adjoint flux $\phi^{+}$. Since $\phi$ is the forward flux, this means that in solving Eq. (169) for $\bar{\psi}$ we must require $\bar{\psi}$ to satisfy the same boundary conditions as $\phi^{+}$(for example, $\bar{\psi}=0$ for outgoing directions at a vacuum boundary). Again we have used only half the contributon flux boundary conditions in solving Eq. (169) for $\bar{\psi}$, namely those obtained from the boundary conditions for $\phi^{+}$. The fact that $\bar{\psi}$ also satisfles the complete set of contributon boundary conditions and is the contributon flux, $\psi=\phi \phi^{+}$, is guaranteed by the relationship in Eq. (170) only because $\phi$ is the forward flux.

In obtaining $\mathrm{Eq}$. (167) from the forward Boltzmann equation [Eq. (164)] it is not necessary that $\phi^{+}$be the adjoint flux, but $\psi$ is not the contributon flux unless $\phi^{+}$is the adjoint flux. Nevertheless, for any well-behaved function $\phi^{+}$, Eq. (168) is still valid for the forward flux $\phi$ if $\frac{\psi^{+}}{\phi^{+}}$satisfies the boundary conditions appropriate for $\phi$. On the other hand, in obtaining Eq. (169) from the adjoint Boltzmann equation [Eq. (45)], it is not necessary that $\phi$ be the forward flux, but $\vec{\psi}$ is not the contributon flux unless $\phi$ is the forward flux. Nevertheless, for any well-behaved function $\phi$, Eq. (170) is still valid for the adjolnt flux $\phi^{+}$if $\frac{\Psi}{\phi}$ satisfles the boundary conditions appropriate for $\phi^{+}$. If, however, we do Identify the $\phi^{+}$in Eq. (167) as the adjoint flux, then the adjo, it Boltzmann equation [Eq. (45)] can be used to reduce Eq. (I67) to

$$
\underline{\Omega} \cdot \nabla \psi+\left(\frac{s^{+}}{\phi^{+}}\right) \psi=S \phi^{+} .
$$

If we idencify the $\phi$ In Eq. (169) as the forward flux, then the forward Boltzmann equation [Eq. (164)] can be used to reduce Eq. (169) to

$$
-\underline{\Omega} \cdot \nabla \bar{\psi}+\left(\frac{s}{\phi}\right) \bar{\psi}=s^{+} \phi .
$$

Inlike Eq. (167), which is valid for any wellbehaved function $\phi^{+}$, it is imperative that the $\phi^{+}$ in Eq. (171) satisfy the adjoint Boltzmann equation with source $s^{+}$, and unlike Eq. (169) which is valid for any well-behaved function $\phi$, it is imperative that the $\phi$ in Eq. (172) satisfy the forward Boltzmann equation with source $S$. This is because $\sigma$ no longer appears in Eqs. (171) and (172), so the only way the solutions, $\psi$ and $\bar{\psi}$, can be influenced by $\sigma$ is through the proper relationships being established between $S$ and $\phi$ and between $\mathrm{S}^{+}$and $\phi^{+}$. 
2. The Iterative Algorithm and Its Properties

The intention is to iterate between the equa-

tions,

$$
-\underline{\Omega} \cdot \nabla \bar{\psi}+\left(\frac{S}{\phi}\right) \bar{\psi}=S^{+} \phi
$$

and

$$
\Omega \cdot \nabla \psi+\left(\frac{s^{+}}{\phi^{+}} \psi=s \phi^{+},\right.
$$

where the $\phi$ in Eq. (173) must satisfy

$$
\underline{\Omega} \cdot \nabla \phi+\sigma^{\dagger}=\mathrm{S}
$$

and the $\phi^{+}$in Eq. (174) must satisfy

$$
-\underline{\Omega} \cdot \nabla \phi^{+}+\sigma \phi^{+}=s^{+}
$$

to insure that $\psi$ and $\bar{\psi}$ are forced to converge to the contributon flux. Thus, the following algorithm will be used:

(1) obtain an estimate $\phi^{+(1)}$ satisfying

$$
-\underline{\Omega} \nabla \phi^{+(1)}+\sigma \phi^{+(1)}=s^{+(0)}
$$

with the usual adjoint flux boundary conditions on $\phi^{+(1)}$;

(2) obtain an estimate $\phi^{(1)}$ satisfying

$\underline{\Omega} \cdot \nabla \phi(1)+\sigma \phi(1)=\mathrm{s}^{(0)}$

with the usual forward flux boundary conditions on $\phi^{(1)}$;

(3) set $k=2$;

(4) solve

$-\Omega \cdot \nabla \bar{\psi}^{(k)}+\left[\frac{s^{(k-2)}}{\phi(k-1)}\right] \bar{\psi}^{(k)}=s^{+(k-1)} \phi(k-1)$

with adjoint flux boundary conditions on $\bar{\psi}^{(k)}$;

(5) set

$$
\phi^{+(k)}=\frac{\bar{\psi}^{(k)}}{\phi^{(k-1)}} ;
$$

(6) solve

$$
\underline{\Omega} \cdot \bar{\psi}{ }^{(k)}+\left[\frac{s^{+(k-1)}}{\phi^{+(k)}}\right] \psi(k)=s^{(k-1)} \psi^{+(k)}
$$

with forward flux boundary conditions on $\psi^{(\mathrm{k})}$;

(7) $\mathrm{se}^{*}$

$$
\phi^{(k)}=\frac{\psi^{(k)}}{\phi^{+(k)}} ;
$$

(8) increase $\mathrm{k}$ by 1 ; and

(9) if a specified convergence criterion for 4 is not satisfied, return to step (4); otherwise stop.

The convergence properties of this scheme are easily determined. Due to the nature of the transformation, $\phi^{(k)}$ is the solution to

$$
\underline{\Omega} \cdot \nabla \phi^{(k)}+\sigma \phi(k)=s^{(k-1)}
$$

and $\phi^{+(k)}$ is the solution to

$$
-\underline{\Omega} \cdot \nabla \phi+(k)+\sigma \phi^{+(k)}=s^{+(k-1)} \text {. }
$$

Consequently, the convergence of $\phi^{(k)}$ and $\phi^{+(k)}$ independently is the same as in the unaccelerated Neumann series solution. ${ }^{14}$ There should be no great difficulty in accelerating the scheme by applying a standard acceleration techrí... to Eqs. (1B3) and (184). What is not clear, however, is whether the scheme could be accelerated by applying some acceleration technique directly to Eqs. (179) and (181).

3. The Numerical Execution of the Iterative Approach - The Problem of Consistency

Because the contributon balance equation [Eq . (46)] is just a combination of the forward and adjoint Boltzmann equations [Eqs. (44) and (45)], only two of these three transport equations are Independent. Since the three flux solutions, $\phi, \phi^{+}$, and $\psi$, are related according to Eq. (5), only two of these three flux solutions are independent. It is, thus, possibie to obtain all three flux distributions by solving for any two using the appropriate pair of transport equations in which the third flux distribution has been eliminated. This third flux can then be obtalned from the two known flux solutions 
through use of Eq. (5). When the flux distributions are treated as continuous functions of the phasespace variables, the three flux solutions obtalned In this manner are conalstent; that is, they are independent of which pair of transport equations are solved. However, when these transport equations are differenced in the space and direction variables, this consistency may be lost unless care is taken in the differencing. A simple example w11 suffice to demonstrate the necessity of a consistent set of difference equations.

Consider the transformed adjoint equation [Eq. (179)] in slab geometry and assume that diamond differencing is applied. Then for cell 1 and direction $\mathrm{m}$, the center-point flux $\bar{\psi}_{\mathrm{ml}}^{(\mathrm{k})}$ is

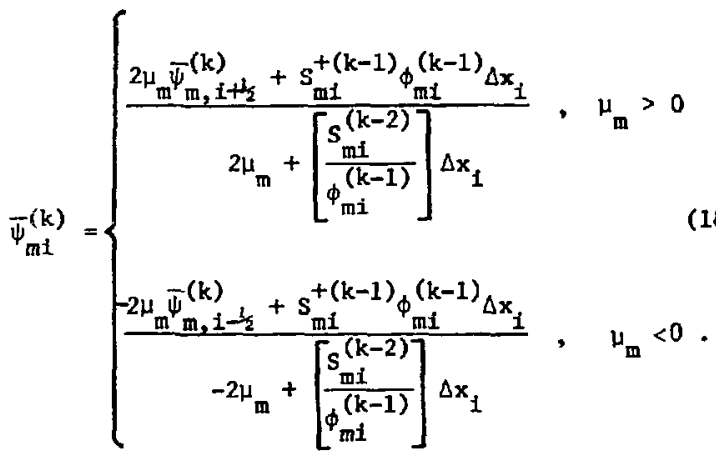

Since $\phi^{+(k)}=\frac{\bar{\psi}^{(k)}}{\phi^{(k-1)}}$, we obta1n 1ts discretized
value,

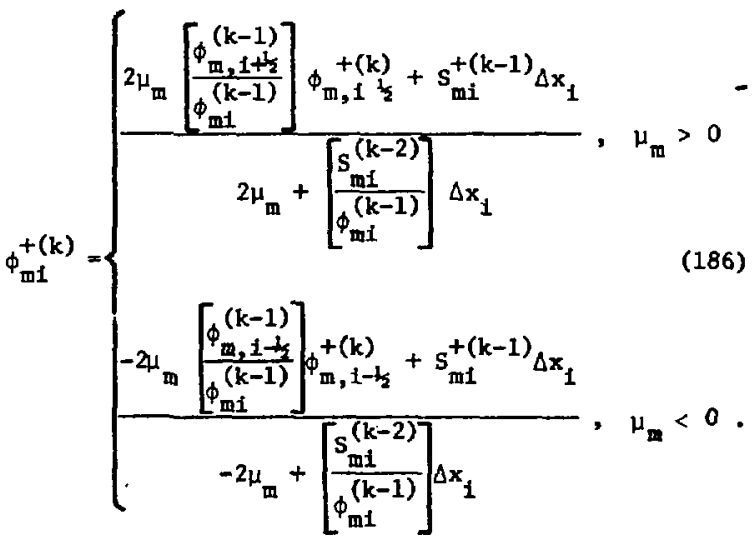

If diamond differencing is applied to the forward Boltzmann equation [Eq. (183)] with $k$ replaced by $k-1$, then

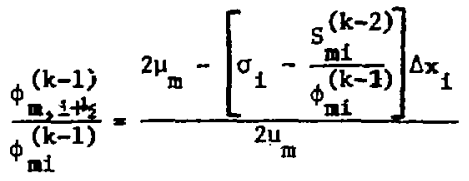

and

$$
\frac{\phi_{m, 1-i / 2}^{(k-1)}}{\phi_{m i}^{(k-1)}}=\frac{2 k_{m}+\left[\sigma_{1}-\frac{s_{m i}^{(k-2)}}{\phi_{m i}^{(k-1)}}\right] \Delta x_{i}}{2 \mu_{m}} .
$$

If these results are then used in Eq. $(186), \phi_{\mathrm{mI}}^{+(k)}$ is given by

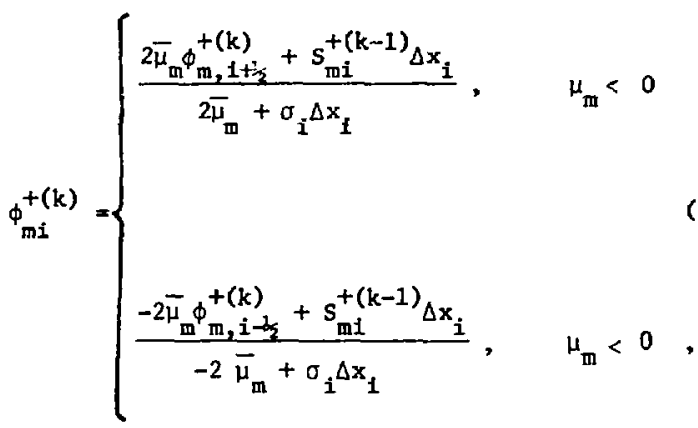

where

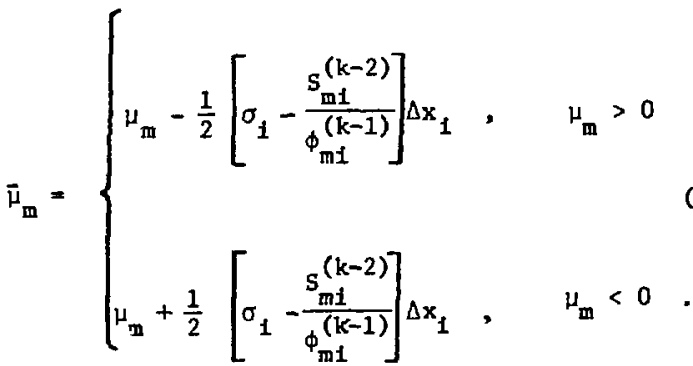

The expressions for $\phi_{\mathrm{ml}}^{+(\mathrm{k})}$ in $\mathrm{Eq} .(189)$ are the usual diamond difference results except for the direction cosines $\bar{\mu}_{m^{*}}$ Since, In general,

$$
\frac{s_{m 1}^{(k-2)}}{\phi_{m 1}^{(\bar{k}-1)}} \neq \sigma_{i}
$$

the directions with cosines $\bar{\mu}_{m}$ will not be the same as the directions with cosines $\mu_{m}$ unless $\Delta x_{1}=0$. 
Thus, an error has apparently been introduced by inconsistent differencing.

Our numerical results support the above conclusion. In these calculations, diamond differencing was used for the $\psi, \bar{\psi}, \phi$, and $\phi^{+}$equations. Initialiy, the convergence was similar to that of the unaccelerated Neumanu series iteration. However, a slight error, apparently introduced by the inconsistency, persisted. This error was of the order of $3 \%$ in the contributon angular flux $\psi$ for a problem with a scattering-to-total ratio of 0.1 and a distance of 5.0 mean-free paths between source anc. detector, and it appeared as a nonsymetry in the spatial distribution of $\psi$ for a symetrir problem. Since the contributon problem is effectively a pure scattering problem, outside the detector region, it might be expected that the errer in $\psi$ would consist of two parts, a rapidly decaying part characteristic of the unaccelerated Neumann series iterations and an extremely slowly decaylng part characteristic of pure scattering problems. However, since the flux distortion remained unchanged after 3000 iterations, it is felt that the problem is not a general lack of convergence but rather that the distorted flux is the true solution to an inconsistent set of difference equations. Numerical results show that when the difference equations are made perfectly consistent, as described in the next section, the persistent error is not introduced.

4. The Numerical Execution of the Iterative Approach - A Consistent Set of Difference Equactions

A general analysis of the discrete-ordinates difference equations corresponding to Eqs. (173)-(176) will now be presented to show how consistency can be obtained. Throughout the analysis strict identity is required whenever two results are required to be the same. However, this restriction could be relaxed somewhat without destroying consistency.

Consider first the problem of calculating a new iterate $\phi^{(k)}$ from known values of $s^{(k-1)}$ and $\phi^{+(k)}$ by the use of the transformed forward equation [Eq. (174)]. The discrete-ordinates balance equations which correspond to Eqs. (174)-(176) are:

$$
\mu_{m}\left[\phi_{m, i+\frac{1}{2}}^{(k)}-\phi_{m, i-\frac{1}{2}}^{(k)}\right]+\sigma_{i} \phi_{m i}^{(k)}=S_{m i}^{(k-1)} \Delta x_{i},
$$

$$
-\mu_{m}\left[\phi_{m, i+\frac{1}{2}}^{+(k)}-\phi_{m, i-\frac{l}{2}}^{+(k)}\right]+\sigma_{i} \phi_{m i}^{+(k)}=s_{m i}^{+(k-1)} \Delta x .
$$

and

$$
\begin{aligned}
& \mu_{m}\left[\psi_{m, i+\frac{1}{2}}^{(k)}-\psi_{m, i-\frac{1}{2}}^{(k)}+\gamma_{m 1}^{+(k)}\left[\frac{s_{m i}^{+(k-1)}}{\phi^{+(k)}}\right]_{m i}^{(k)} \Delta x_{i}\right. \\
& =\gamma_{m i}^{(k)} s_{m i}^{(k-1)_{\phi}+(k)} \Delta x_{i},
\end{aligned}
$$

where $\gamma$ and $\gamma^{\dagger}$ are as yet arbitrary weight factors which must be determined to insure consistency. In these three equations $\phi_{m i}^{(k)}, \phi_{m i}^{+(k)}, s_{m i}^{(k-1)}$, and $s_{m i}^{+(k-1)}$ are cell-averaged quantities, and $\phi_{\left(\mathrm{m}, i \pm \frac{1}{2},\right.} \phi_{\mathrm{m}, \mathrm{i}+\frac{1}{2}}^{+(k)}$, and $\psi_{\mathrm{m}, \mathrm{i} \pm \downarrow_{2}}^{(\mathrm{k})}$ are cell boundary fluxes. Addicional equations which must be consistent are the definitions of the contributon flux,

$$
\psi_{\mathrm{mi}}^{(\mathrm{k})}=\phi_{\mathrm{mi}}^{(\mathrm{k})} \phi_{\mathrm{mi}}^{+(k)}
$$

from the cell-averaged quantities and

$$
\psi_{m, i \pm \frac{1}{2}}^{(k)}=\phi_{m, i \pm \frac{1}{2}}^{(k)} \phi_{m, i \pm \frac{1}{2}}^{+(k)}
$$

from the cell-boundary values. It should be noted that $\psi_{m i}^{(k)}$ is not the cell-averaged contributon flux but is merely, as the equation states, the product of the cell-averaged quantities, $\phi_{\mathrm{mi}}^{(k)}$ and $\phi_{\mathrm{mi}}^{+(k)}$. In other words, Eq. (195) is consistent with the statement that the center-point value of $\psi$ is the product of the center-poins values of $\phi$ and $\phi^{+}$. Finally, since the discrete-ordinates balance equations, Ers. (192)-(194), contafn too many unknown flux values, we must introduce a set of discreteordinates supplementary equations which express the cell-averaged flux value in tems of the cell-boundary flux values, and we require that these also be consistent. For these supplementary equations, we use very general weighted-difference equations in which the welghts are allowed to be functions of $\psi, \phi$, and $\phi^{+}$. The supplementary equations for the forward and adjoint balance equations are, respectively,

$$
\phi_{m i}^{(k)}=a_{m i}^{(k)} \phi_{m, i+\frac{1}{2}}^{(k)}+b_{m i}^{(k)} \phi_{m, i-\frac{k}{2}}^{(k)}
$$




$$
\phi_{m i}^{+(k)}=a_{m 1}^{+(k)} \phi_{m, 1+\frac{k}{2}}^{+(k)}+b_{m 1}^{+(k)} \phi_{m, 1-\frac{1}{2}}^{+(k)}
$$

For $\psi$ it is posstble co obtain two supplementary

equations, one from multiplying Eq. (197) by $\phi_{-(k)}^{+(k)}$ and the second from multiplyfing Eq. (198) by $\phi(\mathbf{k})$.

These two equations are

$$
\psi_{m 1}^{(k)}=a_{m 1}^{(k)}\left[\frac{\psi_{m 1}^{+(}+\cdots}{\phi_{m, 1+\frac{1}{2}}^{+(k)}}\right] \psi_{m, 1+\frac{1}{2}}^{(k)}+b_{m 1}^{(k)}\left[\frac{\phi_{m-1}^{+(k)}}{\phi_{m, 1-\frac{1}{2}}^{+(k)}} \psi_{m, 1-\frac{1}{2}}^{(k)}\right.
$$

and

$$
\psi_{m 1}^{(k)}=a_{m i}^{+(k)}\left[\frac{\phi_{m i}^{(k)}}{\phi_{m, 1+\frac{1}{2}}^{(k)}}\right] \psi_{m, 1+t_{2}}^{(k)}+b_{m 1}^{+(k)}\left[\frac{\phi_{m 1}^{+(k)}}{\phi_{m, 1-\frac{1}{2}}^{+(k)}} \psi_{m, 1-\frac{1}{2}}^{(k)} .\right.
$$

Equations (192) and (197) give, after multiplication by $\phi_{m i}^{+(k)}$,

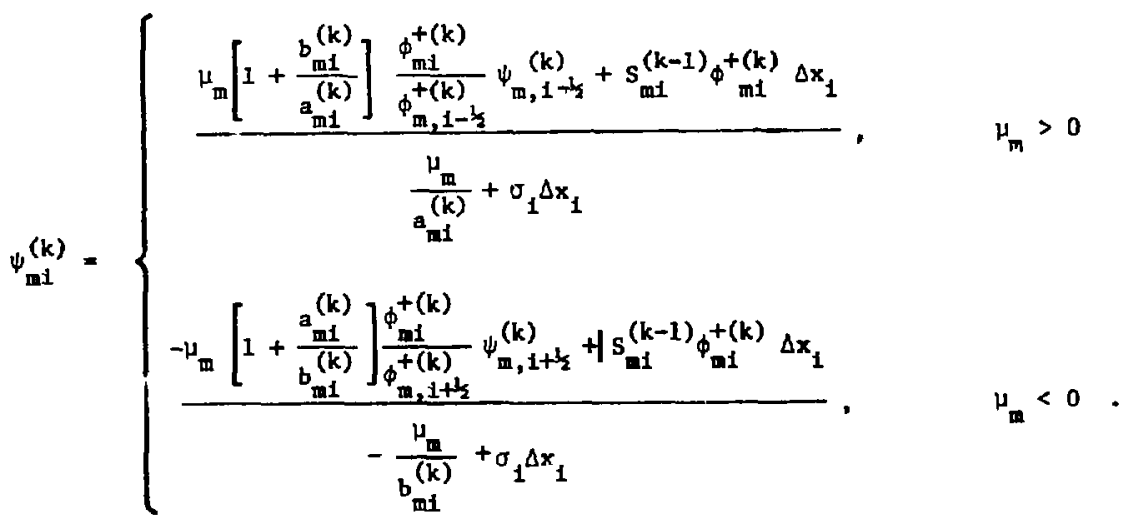

But Eqs. (194) and (199) give 


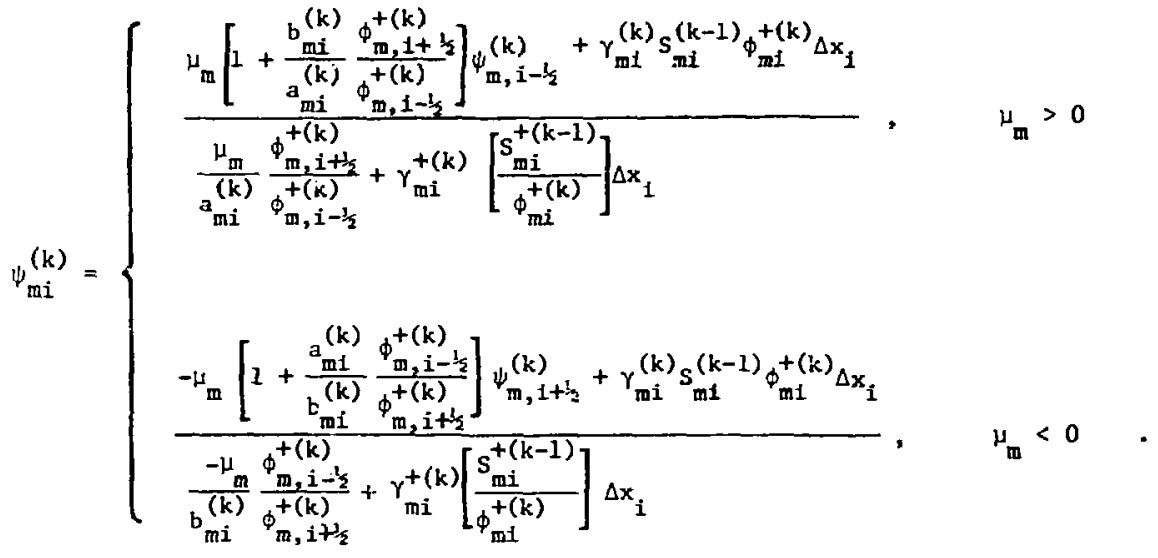

From these two different expressions for $\psi_{\mathrm{mi}}^{(k)}$ we can derive a quantitative requirement for consistency by requiring the identity of both expressions. Two conditions emerge for all directions $\mathrm{m}$ :

$$
-\mu_{m}\left[\phi_{m, i+\frac{1}{2}}^{+(k)}-\phi_{m, i-\frac{1}{2}}^{+(k)}\right]+\sigma_{i}\left[a_{m 1}^{(k)} \phi_{m, i-\frac{1}{2}}^{+(k)}+b_{m i}^{(k)} \phi_{m, i+\frac{1}{2}}^{+(k)}\right] \Delta x_{i}=Y_{m i}^{+(k)}\left[a_{m i}^{(k)}+b_{m i}^{(k)}\right] s_{m i}^{+(k-1)}{ }_{\Delta x_{1}}
$$

and

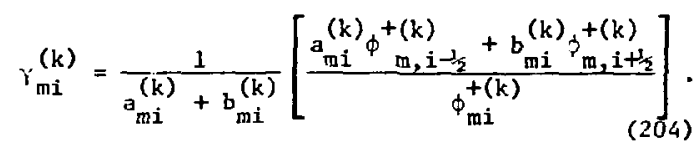

Since we wish the $\phi^{+(k)}$ which is used in Eq. (194) to be the solution to the adjoint difference equation [Eq. (193)], we also obtain

$$
\begin{aligned}
& a_{m i}^{(k)}=b_{m i}^{+(k)}, \\
& b_{m i}^{(k)}=a_{m i}^{+(k)},
\end{aligned}
$$

and

$$
\gamma_{m i}^{+(k)}=\frac{1}{a_{m i}^{(k)}+b_{m i}^{(k)}}
$$

from the requirement that Eqs. (203) and (193) be identical. The use of Eqs. (205), (206), and (198) in Eq. (204) gives

$$
Y_{m i}^{(k)}=\frac{1}{a_{m i}^{(k)}+b_{m i}^{(k)}}=Y_{m i}^{+(k)}
$$

Since $\phi^{+(k)}$ also satisfies Eq. (198) and since Eq. (200) was obtained from Eq. (198), Eqs. (199) and (200) are required to be fdentical, Thus,

$$
\begin{aligned}
& \underset{m i}{(k)}\left[\frac{\phi_{m i}^{+(k)}}{\phi_{m, i+\frac{1}{2}}^{+(k)}}\right]=a_{m i}^{+(k)}\left[\frac{\phi_{m i}^{(k)}}{\phi_{m, i+\gamma_{2}}^{(k)}}\right] \\
& =b_{m-1}^{(k)}\left[\frac{\phi^{(k)}}{\phi_{m, i+\frac{1}{2}}^{(k)}}\right] \\
& =b_{m i}^{+(k)}\left[\frac{\left[\phi_{m i}^{(k)}\right]^{2}}{\phi_{m, 1+d_{s}}^{(k)} \phi_{m, 1-\frac{1}{k}}^{(k)}}\right] /\left[\frac{\phi_{\mathrm{mi}}^{+(k)}}{\phi_{\mathrm{a}, 1-\frac{1}{2}}^{+(k)}}\right]
\end{aligned}
$$

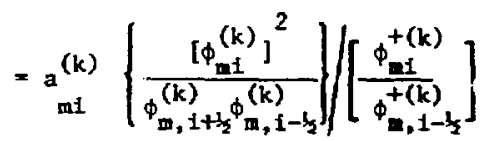


$\frac{\left[\phi_{m i}^{(k)}\right]^{2}}{\phi_{m, i+\frac{1}{2}}^{(k)} \phi_{m, i-\frac{1}{2}}^{(k)}}=\frac{\left[\phi_{m i}^{+(k)}\right]^{2}}{\phi_{m, i+\frac{1}{2} \phi_{m, i-\frac{1}{2}}}^{+(k)}}$

Up to this point we have considered only the solution of the transformed forward Boltzmann equation. We now consider the problem of calculating a new iterate $\phi^{+(k)}$ from known values of $s^{+(k-1)}$ and $\phi^{(k-1)}$ by use of the transformed adjolnt equation [Eq. (173)]. The discrete-ordinates equations which are required to be consistent are

$\mu_{\mathrm{m}}\left[\phi_{\mathrm{m}, i+\frac{1}{2}}^{(k-1)}-\phi_{\mathrm{m}, i-l_{\mathrm{i}}}^{(k-1)}\right]+\sigma_{1} \phi_{\mathrm{mi}}^{(k-1)} \Delta \mathrm{x}_{\mathrm{i}}=\mathrm{s}_{\mathrm{m} 1}^{(k-2)} \Delta \mathrm{x}_{1}$, that

$$
a_{m i}^{+(k)}=b_{n i}^{(k-1)}
$$

$$
b_{n i}^{+(k)}=a_{m i}^{(k-1)}
$$

$\beta_{\mathrm{mi}}^{(k)}=B_{\mathrm{mi}}^{+(k)}=\frac{1}{a_{\mathrm{mi}}^{+(k)}+b_{\mathrm{mi}}^{+(k)}}$

and

$$
\frac{\left[\phi_{n i}^{(k-1)}\right]^{2}}{\phi_{m, 1+l_{2}}^{(k-1)} \phi_{m, 1-\frac{1}{2}}^{(k-1)}}=\frac{\left[\phi_{m i}^{+(k)}\right]^{2}}{\phi_{m, 1+l_{2}}^{+(k)} \phi_{m, 1-\frac{1}{2}}^{+(k)}}
$$

From Eqs. (210) and (223), we see that

$-\mu_{m}\left[\phi_{m, 1+\frac{1}{2}}^{+(k)}-\phi_{m, 1-1 / 2}^{+(k)}\right]+\sigma_{i} \phi_{m 1}^{+(k)} \Delta_{x_{1}}=s_{m i}^{+(k-1)} \Delta_{x_{1}}$,

$-\mu_{m}\left[\bar{\psi}_{m, i+\frac{1}{2}}^{(k)}-\bar{\psi}_{m, i-\frac{1}{2}}^{(k)}\right]+\beta_{m i}^{(k)}\left[\frac{S_{m i}^{(k-2)}}{\phi_{m i}^{(k-1)}}\right] \bar{\psi}_{m i}^{(k)} \Delta x_{i}=B_{m i}^{+(k)} s_{m i}^{+(k-1)} \phi_{m 1}^{(k-1)} \Delta x_{I}$,

$\bar{\psi}_{\mathrm{mi}}^{(k)}=\phi_{\mathrm{mi}}^{(k-1)} \phi_{\mathrm{mi}}+(k)$

$\bar{\Psi}_{m, i \pm_{z}}^{(k)}=\phi_{m, i \pm_{2}}^{(k-1)} \phi_{m, 1 \pm_{s}}^{+(k)}$,

$\phi_{m i}^{(k-1)}=a_{m i}^{(k-1)} \phi_{m, 1+\frac{1}{2}}^{(k-1)}+b_{m 1}^{(k-1)} \phi_{m, i-\frac{1}{2}}^{(k-1)}$,

$\phi_{m I}^{+(k)}=a_{m i}^{+(k)} \phi_{m, i+k_{1}}^{+(k)}+b_{m \perp}^{+(k)} \phi_{m, 1-\frac{1}{2}}^{+(k)}$,

and

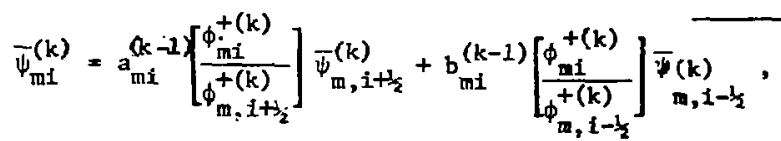

(218)

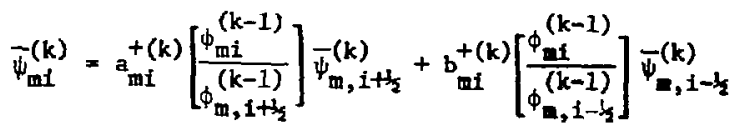




$$
\frac{\left[\phi_{m i}^{(k)}\right]^{2}}{\phi_{m, 1+l_{2}}^{(k)} \phi_{m, 1-\frac{1}{2}}^{(k)}}=\frac{\left[\phi_{m i}^{+(k)}\right]^{2}}{\phi_{m, i+x_{2}}^{(k)} \phi_{m, 1-\frac{1}{2}}^{(k)}}=c_{m i}^{2},
$$

where $c_{m i}$ is independent of $k$. However, since all the fluxes on the seft-hand sile of Eq. (225) are unknown, $C_{m i}$ is also unknown. To implement the iterative solution technique, $C_{m i}$ must be predetermined in some manner. One possibility is to assume a flux shape within the spatial cell and then to determine $C_{m i}$ by inserting these fluxes inro Eq. (225). Since we do not know the exact flux solution a priori, we must attempt to make the best possible assumption for the flux shape within the cell. From Eqs. (205), (206), (220), and (221), we see that the quantities, $a_{m i}=b_{m i}^{+}$and $b_{m i}=a_{m i}^{+}$, are also independent of $k$. It kewise,

$$
Y=Y^{+}=B=B^{+}
$$

independent of $k$.

The consistent forms of the transformed forward and transformed adjoint equations can now be determined. From Eqs. (197), (198), (220), and (221), we obtain

$$
a_{m 1} \phi_{m, i+\frac{1}{2}}^{(k)}+b_{m i} \phi_{m, i-\frac{1}{2}}^{(k)}=\phi_{m i}^{(k)}
$$

and

$$
a_{m i} \phi_{i u, i-l_{2}}^{+(k)}+b_{m i} \phi_{m, i+\frac{1}{2}}^{+(k)}=\phi_{m i}^{+(k)} \text {. }
$$

Cross multiplication of these equations shows that

$$
a_{m i}\left[\psi_{m, i+\frac{1}{2}}^{(k)}-\psi_{m, i-\frac{1}{2}}^{(k)}\right]=\frac{\phi_{m, i+\frac{1}{2}}^{+(k)}}{\phi_{m i}^{+(k)}} \psi_{m i}^{(k)}-\frac{\phi_{m i}^{+(k)}}{\phi_{m, i-\frac{1}{2}}^{+(k)}} \psi_{m, i-\frac{1}{2}}^{(k)}
$$

and

$$
b_{m i}\left[\psi_{m, 1+\frac{1}{2}}^{(k)}-\psi_{m, i-\frac{1}{z}}^{(k)}\right]=\frac{\phi_{m i}^{+(k)}}{\phi_{m, 1+\frac{1}{2}}^{+(k)} \psi_{m, i-\frac{1}{2}}^{(k)}}-\frac{\phi_{m, 1-\frac{1}{2}}^{+(k)}(k)}{\phi_{m i}^{+(k)}} \Psi_{m i} .
$$

If these results and Eq. (208' for $\gamma_{\mathrm{mi}}$ and $\gamma_{\mathrm{mi}}^{+}$are inserted into Eq. (194), the result is

$$
\begin{aligned}
& \mu_{m}\left[\frac{\phi_{m i}^{+(k)}}{\phi_{m, 1+\frac{1}{2}}^{+(k)}} \psi_{m, i+\frac{1}{2}}^{(k)}-\frac{\phi_{m i}^{+(k)}}{\phi_{m, 1-\frac{1}{2}}^{+(k)}} \psi_{m, i-\frac{1}{2}}^{(k)}\right]
\end{aligned}
$$

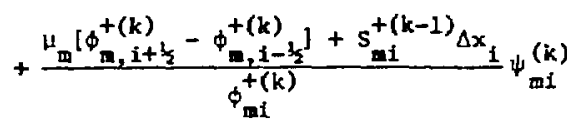

$$
\begin{aligned}
& =s_{m i}^{(k-1)} \phi_{m i}+(k) \Delta x_{i} .
\end{aligned}
$$

Through the use of Eq. (193), this transformed forward equation becomes

$$
\begin{aligned}
& \omega_{m}\left[\frac{\phi_{m i}^{+(k)}}{\phi_{m, i+\frac{1}{2}}^{+(k)}} \psi_{m, i+\frac{1}{2}}^{(k)}-\frac{\phi_{m i}^{+(k)}}{\phi_{m, i-\frac{1}{2}}^{+(k)}} \psi_{m, i-\frac{1}{2}}^{(k)}\right] \\
& +\sigma_{i} \psi_{m 1}^{(k)} \Delta x_{i}=s_{m i}^{(k-1)} \phi_{m i}^{+(k)} \Delta x_{i},
\end{aligned}
$$

which is just $\phi_{\mathrm{mi}}^{+(k)}$ times the forward difference equation [Eq. (192)]. This result insures that $\phi^{(k)}$ satisfies the forward difference equations no matter what $\phi^{+(k)}$ is. Thus, Eq. (231) is more accurately the difference equation corresponding to Eq. (167). A similar analysis shows that the consistent transformed adjoint difference equation is

$$
-\min _{m}\left[\frac{\phi_{m i}^{(k-1)}}{\phi_{m, i+1 / 2}^{(k-1)}} \bar{\psi}_{m, i+\frac{1}{2}}^{(k)}-\frac{\phi_{m i}^{(k-1)}}{\phi_{m, i-\frac{1}{2}}^{(k-1)}} \bar{\psi}_{m, i-l_{2}}\right]
$$


which is just $\phi_{\mathrm{ml}}^{(k-1)}$ times the adjoint difference

Eq. [Eq. (193)]. The consistent supplenentary equation to be used with Eq. (231) is

$$
\left[\psi_{m 1}^{(k)}\right]^{2}=c_{m i}^{4} \psi_{m, 1+\frac{1}{2}}^{(k)} \psi_{m, 1-\frac{1}{2}}^{(k)},
$$

and the suppiewentary equation to be used with Eq.

(232) is

$$
\left[\bar{\psi}_{m 1}^{(k)}\right]^{2}=c_{m 1}^{4} \bar{\psi}_{m, 1+\frac{1}{2}}^{(k)} \bar{\psi}_{m, 1-\frac{1}{2}}^{(k)} .
$$

A number of coments are $f_{n}$ order concerning the supplementary equations for the forward and adjolnt difference equations, namely

$$
\left[\phi_{m 1}^{(k)}\right]^{2}=c_{m 1}^{2} \phi_{m, i+\frac{1}{2} \phi_{m, i-\frac{1}{2}}^{(k)}}^{(k)}
$$

and

$$
\left[\phi_{m 1}^{+(k)}\right]^{2}=c_{m 1}^{2} \phi_{m, 1+k_{2}}^{+(k)} \phi_{m, 1-\frac{1}{2}}^{+(k)} .
$$

These equations are a generalization of the geometric mean, or exponential, supplenentary equatlons. 5 It is shown In Appendix $C$ to this report that allowing $C_{m 1} \neq 1$ is necessary for consistency between the discrete-ordinates balance equation and Its corresponding supplenentary equation. Indeed, the accuracy of the solution can be sensitive to the choice of $\mathrm{C}_{\mathrm{mi}}$, as shown later.

Obviously, the supplementary equations [Eqs. (233)-(236)] are Invalid for certain directions in a cell adjacent to a vacuum boundary. A consistency analysis has been carried out for the special case of a vacuum boundary. Unlike the case of an interlor cell, the requirement of constitency between the two forms of the $\psi$ (or $\bar{\psi}$ ) weighted-difference equations Imposes no additional constraints, and the following supplementary equations are obtained:

(1) for a left boundary cell,

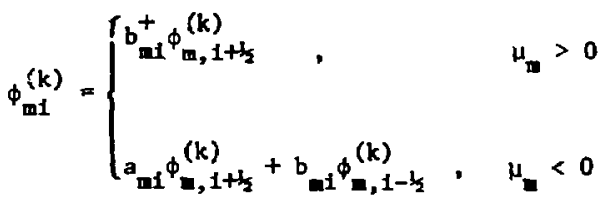

$\phi_{m i}^{+(k)}= \begin{cases}a_{m 1}^{+} \phi_{m, 1+\frac{1}{2}}^{+(k)}+b_{m 1}^{+} \phi_{m, 1-\frac{1}{2}}^{+(k)}, & \mu_{m}>0 \\ b_{m 1} \phi_{m, 1+\frac{1}{2}}^{+(k)}, & \mu_{m}<0 ;\end{cases}$

and

(2) for a right boundary cell,

$$
\phi_{m 1}^{+(k)}= \begin{cases}a_{m 1} \phi_{m, 1+\frac{1}{2}}^{(k)}+b_{m i} \phi_{m, i-\frac{1}{2}}^{(k)}, & \mu_{m}>0 \\ a_{m 1}^{+} \phi_{m, 1-\frac{1}{2}}^{(k)}, & \mu_{m}<0\end{cases}
$$

and

$$
\phi_{m 1}^{+(k)}=\left\{\begin{array}{ll}
a_{m 1} \phi_{m, 1-\frac{1}{2}}^{+(k)}, & \mu_{m}>0 \\
a_{m 1^{+}}^{+} \phi_{m, 1+\frac{1}{2}}^{+(k)}+b_{m 1}^{+} \phi_{m, 1-\frac{1}{2}}^{+(k)}, & \mu_{m}<0 .
\end{array} .\right.
$$

As before, the transformed forward equation is just $\phi_{m i}^{+(k)}$ times the forward difference equation, and the transformed adjoint equation is just $\phi_{\mathrm{mi}}^{(\mathrm{k}-1)}$ times the adjoint difference equation.

5. Numerical Results for the Iterative Approach The intention with this iterative approach is to use coarser spatial meshes for $\psi$ and $\bar{\psi}$ than for $\phi$ and $\phi^{+}$based upon the expected slower variation of $\psi$ and $\bar{\psi}$. At the beginning of each tteration $:$, informat Ion 18 assumed to be avallable for $(k-1)$ and $\phi^{+(k-1)}$ on a fine spatial wesh. Then the $\bar{\psi}$ equation is solved on a coarse mesh in those reglons where $\bar{\psi}$ is expected to vary nore slowly than $\phi$ and $\phi^{+}$(and on the fine wesh in the remaining reglons). The solution $\bar{\psi}$ on the coarse mesh and the inforation $\phi^{(k-1)}$ on the fine mesh are then used to obtain a new lterate $\phi^{+(k)}$ on the fine mesh. The approximation used in obtaining $\phi^{+(k)}$ in this manner is that $\bar{\psi}$ varies exponentially across the coarse-mesh cell, which is consistent with the supplementary equation for $\bar{\psi}$, Eq. (234). To complete the iteration k, a similar approach $1 s$ then taken with the $\psi$ equation and the calculation of the new iterate $\phi^{(k)}$.

Every attenpt has been inade to maintain consistency ang the equations. This is easily done 
when the coarse and ine meshes colncide, and for such a case the convergence of the iterations is similar to that of the unaccelerated Neumann serfes iterations. However, when the coarse and fine meshes do not coincide, an inconsistency and its accompanying persistent error are introduced again. Thus, some modification of this technique appears 20 be necessary in the future if the slow variation in $\psi$ is to be exploited without loss of consistency.

The results for a simple scattering problem will now be described, since they provide additional insight into the physics of contributon transport. In these firs: calculations, the iterative approach described in the previous sections has been employed with coinciding fine and coarse meshes. The geometry and important parameters for this problem are shown in Fig. 9, and a plot of $\psi(x, \mu)$ for the $S_{2}$ approximation is shown in Fig. 10. The interesting details in this plot are the appearance of the peaks in the source and detector regions for $\mu<0$ and the bebehavior of $\psi$ in the central region.

In the central region of the slab the angular contributon flux distritution $\psi$ varies for each of the two directions in such a way that the difference between the $\psi$ values remains constant. This is a direct consequence of the conservation law, ${ }^{2}$

$$
\iiint_{V} \underline{\Omega} \cdot \nabla \psi(\underline{\mathbf{r}}, \underline{\Omega}, \mathrm{E}) \mathrm{d} \underline{\Omega} \mathrm{dE} \mathrm{d} \psi=0,
$$

where $V$ is any volume which does not include the source or detector. Eq. (241), when applied to our

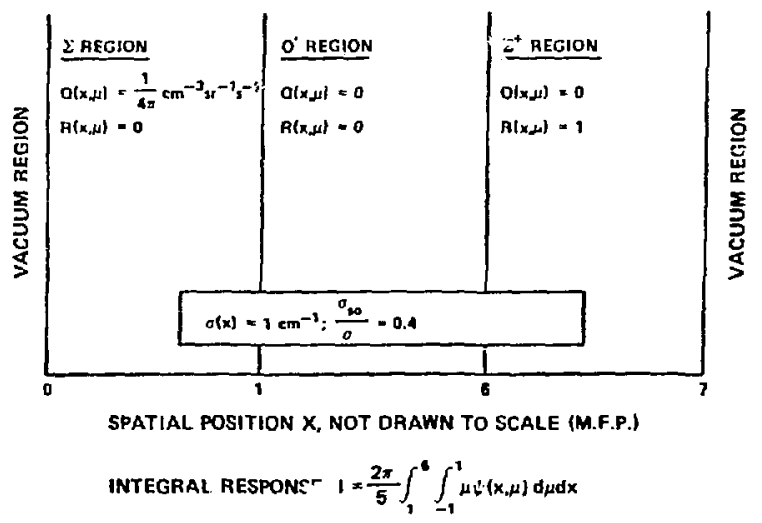

Fig. 9. Geometry for model scattering problem. spectal $\mathrm{S}_{2}$ problem and integrated over any volume in the $a^{\prime}$ region becomes

$$
\psi\left(x_{2}, \mu_{2}\right)-\psi\left(x_{2}, \mu_{1}\right)=\psi\left(x_{1}, \mu_{2}\right)-\psi\left(x_{1}, \mu_{1}\right)
$$

where $x_{1}$ and $x_{2}$ are any two spatial positions in the $O^{\prime}$ region and the two direction cosines are related by

$$
\mu_{2}=-\mu_{1}
$$

Thus, in this simple two-direction case, any contributon picked up by a contributon "packet" traveling in one direction must be lost by the contributon "packet" traveling in the other direction.

The peaks in the source and detector regions are due mainly to the presence of the source and detector and to the fact that the vacuum boundaries act as perfect reflectors of contributons. In the source region, 'te packet of contributons with $\mu>$ 0 picks up contributons produced by the source, causing the rise in $\psi$ after the pack't enters the source region. However, all the contributons in the packet must be scattered into the direction with $\mu>0$ before the vacuum boundary is encountered, and this results in the decrease in $\varphi$ as the vacuum boundary is approached. In the detector region, the situation is reversed. There the packet of contributons with $\mu<0$ picks up contributons sicattered from the direction with,$>0$ in the viciniry of tine vacuum boundary. This causes the rise in $\downarrow$ as the packet moves away from the vacuum boundary. The detector, on the other hand, acts as a link for contributons and causes the decrease in $\psi$ as the $0^{*}$ region is approached. Aithough it is not evident from this semi-logarithmic plot, $\psi(x, ;)$ does go to zero $a^{\text {t }}$ the two vacuum boundaries for all directions.

When a multiple grid is used and a certain value is specified for $\mathrm{C}_{\mathrm{mi}}$ in the fine-mesh cells, the question arises of what value to assign to $C_{m j}$ for the coarse-mesh cells. From the discission in Appendix $C$, it is easily seen that $C_{\mathrm{mj}}$ may differ significantly from $C_{m i}$. Two approaches were tried in the calculations for the problea described in Fig. 9. Method 1 consisted of evaluating $\mathrm{C}_{\mathrm{aj}}$ by 


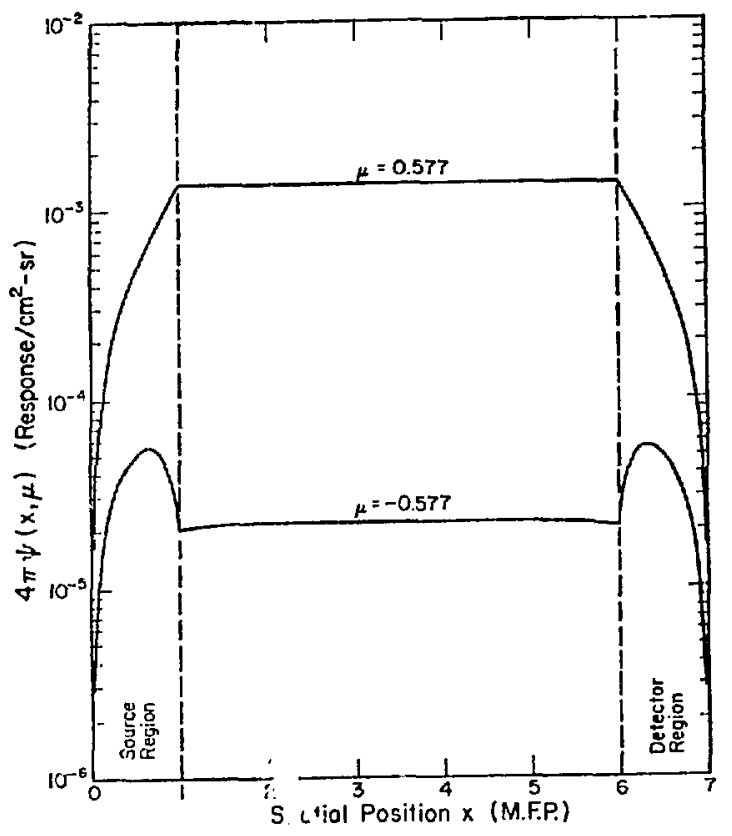

Fig. 10. Solution for model scattering problem in the $\mathrm{S}_{2}$ approximation.

use of Eq. (225) from the fine-mesh forward and adjoint flux data in each coarse-mesh cell in only the first iteration after the relative error between successive iterates of the integral response $I$, computed from Eq. (5:), fell below 0.1. Method 2 consisted in evaluating $c_{m j}$ in a similar manner after every iteration for $k>2$. Figure 11 is a plot of the integral response $I$ vs the size of the coarsemesh cell in the $0^{\prime}$ region. The fine-mesh cell width used with Methods 1 and 2 was 0.1 mean-free paths in the $0^{\prime}$ region and 0.05 mean-free paths in the source and detector regions. The value assigned to $C_{m i}$ for all flne-mesh cells was one and a coarse mesh was used only in the $0^{\prime}$ region. A compartson calculation has also been made for each of the coarse-mesh choices where only thls particular coarse mesh was employed as a single spatial grid with $C_{m 1}=1$. As can be seen, considerable improvement in the integral response is obtained by employing multiple-grid Method 2, in which $c_{m j}$ in the coarse-mesh cells is evaluated from the fine-mesh flux data after each Iteration.

As previously stated, the use of multiple grids introduces distortions into the flux solutions. For the problem described in Fig. 9, the contributon

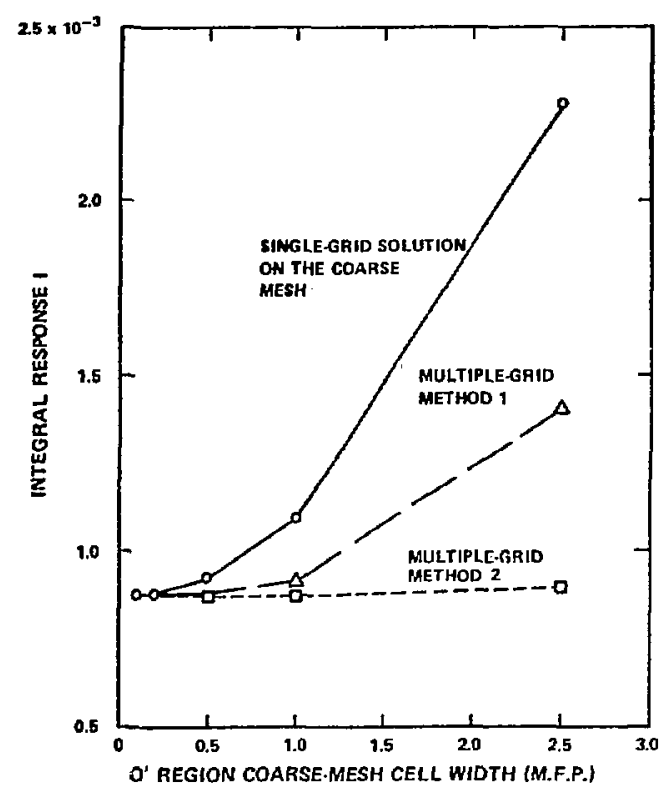

Fig. 11. $\mathrm{S}_{8}$ integral response for the model scattering problem.

total flux should be symmetric. Therefore, one measure of such distortion would be the tilt in the contributon total flux, where the tilt is defined to be

$$
\operatorname{TILT}=\left|1-\frac{\bar{\psi}_{\mathrm{R}}}{\bar{\psi}_{\mathrm{Q}}}\right|,
$$

and $\bar{\psi}_{Q}$ and $\bar{\psi}_{R}$ are the average Integrated contributon fluxes in the source and detector regions. As indicated In Fig. 12, the flux distortion increases with increasing coarse-mesh cell width in the $0^{\prime}$ region but remalns below about $20 \%$ for $\sigma \Delta x \leq 2.5$. Figure 13 presents an extreme example of a distorted contributon angular flux for the case of only two coarse-mesh cells in the entire $0^{\prime}$ region ( $\left.\sigma \Delta x=2.5\right)$. For comparison, the corresponding reference solution on the finest mesh is also plotted. The multiplegrid solution was obtained by using Method 2, and the locations of the coarse-mesh cell boundaries In the $0^{\prime}$ region are readily apparent in the plot of the distorted flux. The relative error, $\mid \psi-$ $\psi_{\text {EXACT }} / \psi_{\text {EXACT }}$ in thfs distorted contributon angular 


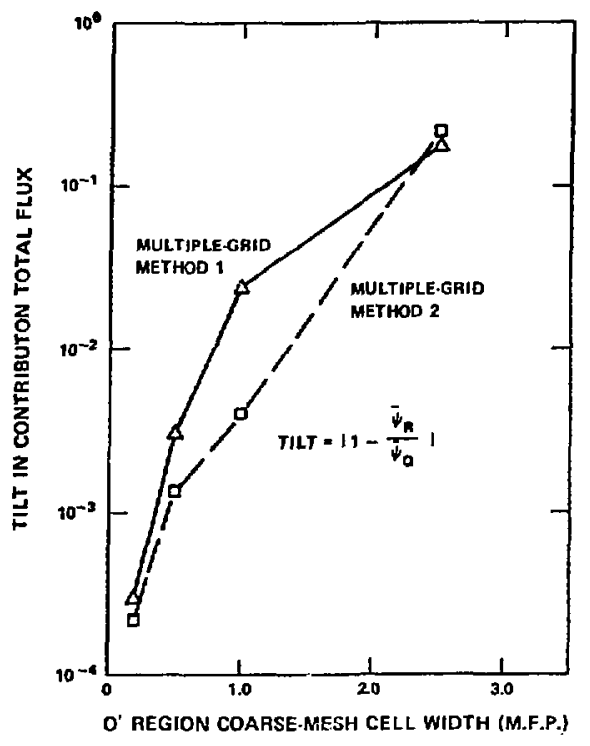

Fig. 12. $S_{8}$ contributon-flux distortion in multiplegrid solution of model : atering problen.

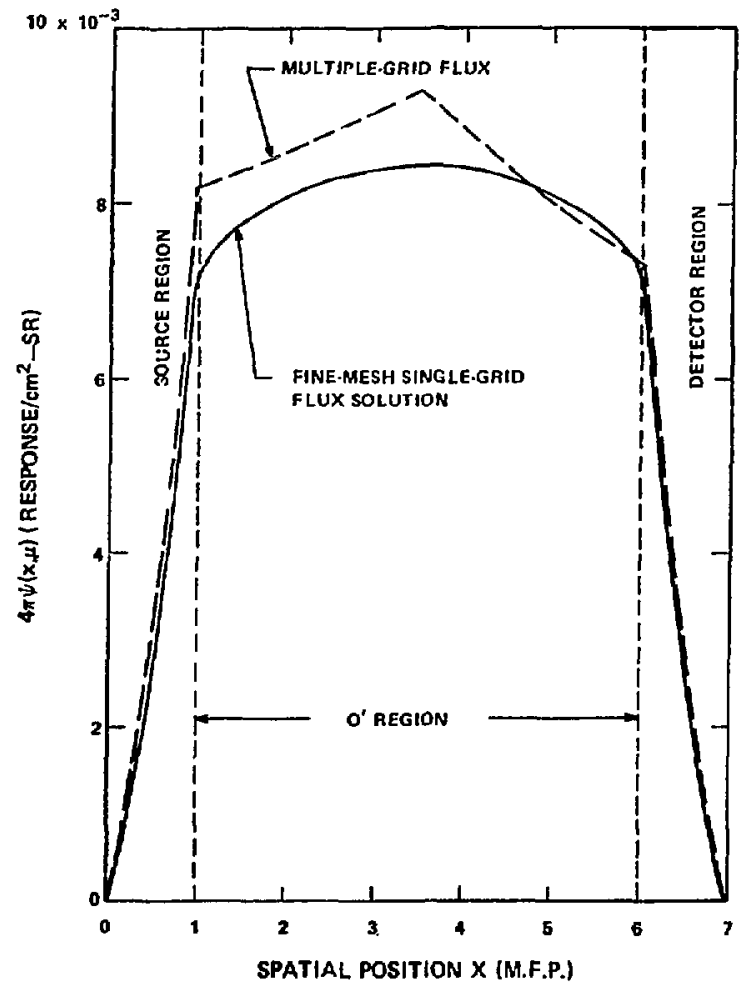

F1g. 13. Comparison of distorted contributon angular flux with reference solution to model scattering problem for $\mu=0.960$. flux is plotted in Fig. 14 and remains telow $12 \%$. For comparison, the relative error when five coarsemesh cells are used in the $0^{\prime}$ region is also plotted. There is a rather dramatic improvement in the accuracy of the solution due to the 1ncrease in the number of coarse-mesh cells. In order to trace these distortions in $\psi$ back to errors in $\phi$ or $\phi^{+}$, we plot in Fig. 15 the relative errors in $\phi$ and $\phi^{+}$as they remain in the fine-mesh results after the iterative process is converged to a relative error of $10^{-6}$ between successtve iterates of the integral response I. From Figs. 14 and 15, a fairly interesting observation can te made. Most of the distortion in the contributon flux is due to the distortion in the fine-mesh adjoint flux. The forward flux is fairly accurate for -11 spatial positions, with errors on the order of $1 \%$ or less.

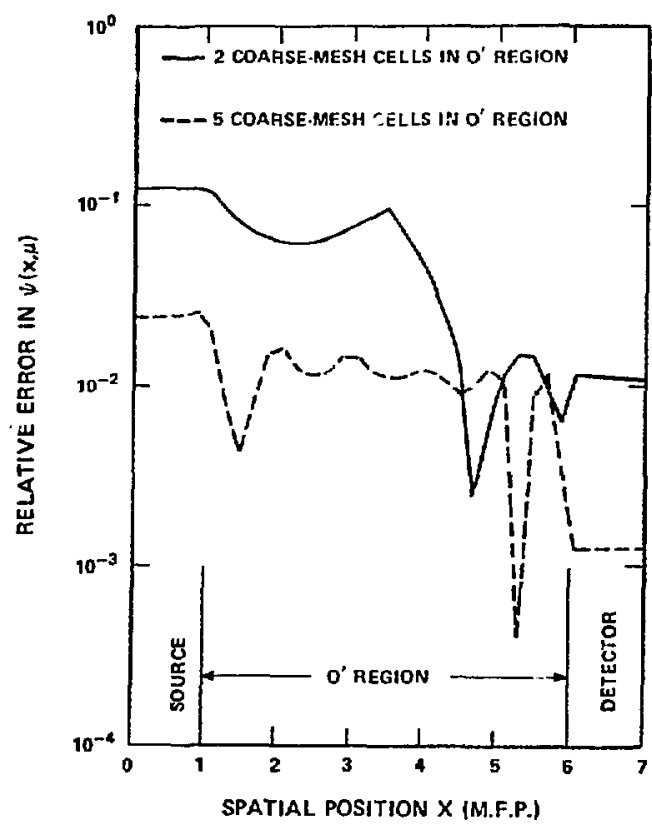

Fig. 14. Error in $s_{8}$ multiple-grid contributon flux solution to model scattering problem for $u=0.960$. 


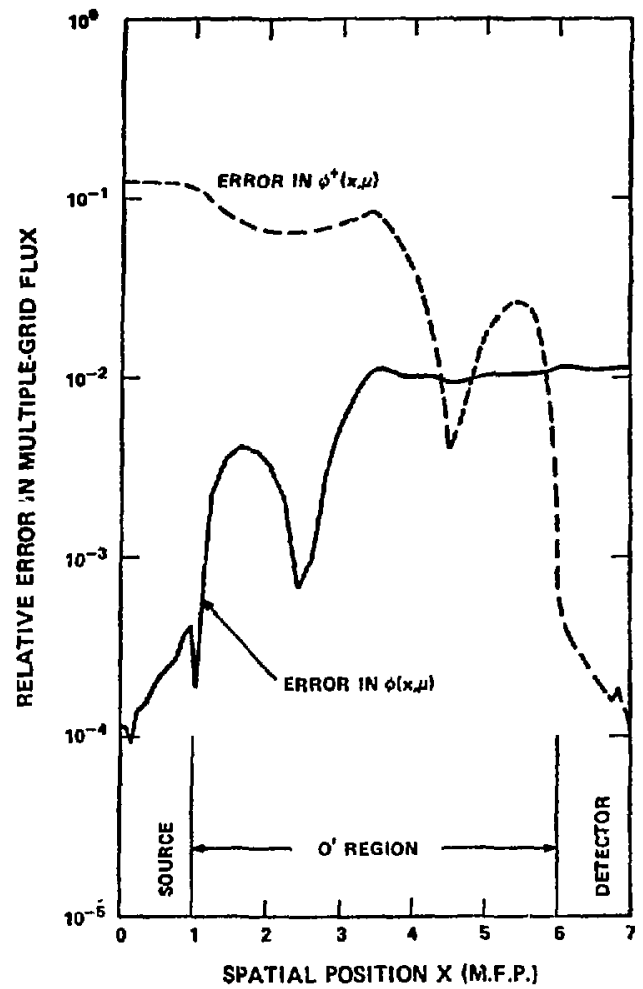

Fig. 15. Error in $\mathrm{S}_{8}$ multiple-grid flux solutions, $\phi(x, \mu)$ and $\phi^{+}(x, \mu)$, to model scattering problem for $\mu=0.960$.

The source of the flux distortions in the mu1tiple-grid approach is in all probability an inconsistency between the discrete-ordinates equations for the fine spatial mesh and the discrete-ordinates equations for the coarse-spatial mesh. However, the nature of this inconsistency is not known at this time, and consequently, it has not besn possible to eliminate the flux distortions in the multiple-grid approach.

D. An Integral Equation Approach to the Contributon Transport Problem

This approach Involves the solution of an integral equation with a generalized contributon flux as the unknown. This approach is not being seriously considered at this time and is tncluded here only because it provides some insight into the information required to solve the contributon transport problem.

The Integral form of the forward Boltzman equation can be written ${ }^{1}$

$\phi(\underline{x})-M\left(\underline{x}, \underline{x}^{\prime}\right) \phi\left(\underline{x}^{\prime}\right)=N\left(\underline{x}, \underline{x}^{\prime}\right) \phi\left(\underline{x}, \underline{x^{\prime}}\right)$, where

$$
\begin{aligned}
& \underline{x} \text { = the phase-space vector }(\underline{x}, \underline{R}, E) \text {, } \\
& \underline{x}^{\prime}=\text { the phase-space vector }\left(\underline{I}-s^{\prime} \underline{\Omega}, \underline{\Omega}, E^{\prime}\right) \text {, } \\
& M\left(\underline{x}, \underline{x}^{\prime}\right)=\int_{0}^{\infty} e^{-f_{0}^{s^{\prime}} \sigma\left(\underline{x}-s^{\prime} \underline{\Omega}, E\right) d s^{\prime \prime}} \\
& \iint \sigma_{s}\left(\underline{I}-s^{\prime} \underline{\Omega} \underline{\Omega}^{\prime}, E^{\prime}+\underline{\Omega}, E\right) \underline{d}^{\prime} d E^{\prime} d s^{\prime} \\
& \text { = the multiple-collision 1ntegral operator, } \\
& N\left(\underline{x}, \underline{x}^{\prime}\right)=\int_{0}^{\infty} e^{-\int_{0}^{s^{1}} \sigma\left(\underline{r}-s^{\prime} \underline{\Omega}, E\right) d s^{\prime \prime}} d s^{\prime} \\
& \text { = the noncollision integral operator, }
\end{aligned}
$$

anc

$$
\begin{aligned}
& Q\left(\underline{\underline{x}}, \underline{\underline{x}}^{\prime}\right)=Q\left(\underline{\mathbf{r}}-\mathbf{s}^{\prime} \underline{\Omega}, \underline{\Omega}, \underline{E}\right) \\
& =\text { external source distribution along the } \\
& \text { direction vector } \underline{\Omega} \text { passing through the } \\
& \text { spatial position } \underline{\underline{r}} .
\end{aligned}
$$

The quantities, $s^{\prime}$ and $s^{\prime \prime}$, are scalar path length variables. Likewise the integral form of the adjoint Boltzmann equation is

$$
\phi^{+}(y)-M^{+}\left(y, y^{\prime}\right) \phi^{+}\left(y^{\prime}\right)=N^{+}\left(y, y^{\prime}\right) R\left(y, y^{\prime}\right)
$$

where

$$
\begin{aligned}
y & =\text { the phase-space vector }(\underline{\rho}, \underline{\omega}, e), \\
y^{\prime} & =\text { the phase-space vector }\left(\underline{\rho}+t^{\prime} \underline{\omega}, \underline{\omega}^{\prime}, e^{\prime}\right), \\
M^{+}\left(y, y^{\prime}\right) & =\int_{0}^{\infty} e^{-\int_{0}^{t^{\prime}} \sigma\left(\underline{p}+t^{\prime \prime} \underline{\omega}, e\right) d t^{\prime \prime}}
\end{aligned}
$$$$
\iint \sigma_{s}\left(\underline{e}+t^{\prime} \underline{\omega} ; \underline{\omega}, e^{+} \underline{\omega}^{\prime}, e^{\prime}\right) d \underline{\omega^{\prime}} d e^{\prime} d t^{\prime},
$$ 


$$
N^{+}\left(y, y^{\prime}\right)=\int_{0}^{\infty} e^{-\int_{0}^{t^{\prime}} \sigma\left(\underline{\rho}+t^{\prime \prime} \underline{\omega}, e\right) d t^{\prime \prime}} d t^{\prime}
$$

and

$R\left(y, \underline{y}^{\prime}\right)=R\left(\underline{\underline{\rho}}+t^{\prime} \underline{\omega}, \underline{\omega}, e\right)$

= detector response function along the direction vector $\underline{\omega}$ passing through the spatial position $Q$.

Again the quantities, $t^{\prime}$ and $t^{\prime \prime}$, are scalar path length variables.

A generalized contributon flux is now defined to be

$$
\Psi(\underline{x}, \underline{y})=\phi(\underline{x}) \phi^{+}(\underline{y})
$$

If the corresponding sides of Eqs. (245) and (249) are multiplied together and the definition in Eq. (253) is used, the result is

$$
\Psi(\underline{x}, \underline{y})=M\left(\underline{x}, \underline{x}^{\prime}\right) \Psi\left(\underline{x}^{\prime}, \underline{y}\right)+M^{+}\left(\underline{y}, \underline{y}^{\prime}\right) \Psi\left(\underline{x}, \underline{y}^{\prime}\right)-M\left(\underline{x}, \underline{x}^{\prime}\right) M^{+}\left(\underline{y}, \underline{y}^{\prime}\right) \Psi\left(\underline{x}^{\prime}, \underline{y}^{\prime}\right)+N\left(\underline{x}, \underline{x}^{\prime}\right) Q\left(\underline{x}, \underline{x}^{\prime}\right) N^{+}\left(\underline{y}, \underline{y}^{\prime}\right) R\left(\underline{y}, \underline{y}^{\prime}\right)
$$

The ordinary contributon Flux, as defined in Eq. (5), is just

$$
\psi(\underline{x})=\psi(\underline{x}, \underline{x})=\phi(\underline{x}) \phi^{+}(\underline{x}) .
$$

Although it may appear that the amount of contributon information has been squared by the introduction of $\Psi$, such is not the case. From its definition in Eq. (253), it follows that $\Psi(\underline{x}, \underline{y})$ can be completely described by just two independent distributions. Any two of the three functions, $\phi(x)$, $\phi^{+}(\underline{x})$, and $\psi(\underline{x})$, are such independent distributions. We now ask whether it is possible to find another distribution $\chi(\underline{x})$, such that $\psi(\underline{x})$ and $\chi(\underline{x})$ are independent, completely determine $\Psi(\underline{x}, \underline{y})$, and vary more slowly in $\underline{I}$ than $\phi(\underline{x})$ and $\phi^{+}(\underline{x})$ over a portion of the system.

To determine how $\chi(\underline{x})$ might be found, consider the special case of a pure absorber 0 region in slab geometry, for which

$$
\phi^{+}(\underline{x})=A^{\phi^{-1}}(\underline{x})
$$

and $A$ is constant in the spatial variable $x$. Since the exponent of $\phi$ will be of importance to the remainder of the discussion, we also write

$$
\phi(x) \equiv \phi^{1}(\underline{x})
$$

and

$$
\psi(\underline{\mathbf{x}})=A \phi^{\circ}(\underline{x})=A .
$$

We can also define a fourth distribution function $\phi_{R}(\underline{x})$, where $\phi_{R}(\underline{x})$ is just a spatial reflection of $\phi(\underline{x})$ about the center plane $x=x_{0}$ of the slab 0 region.* Since this function $\phi_{R}(x)$ is related to $\phi(\underline{x})$ for this special case by the equation

$$
\phi_{R}(\underline{x})=\phi^{-1}(\underline{x}),
$$




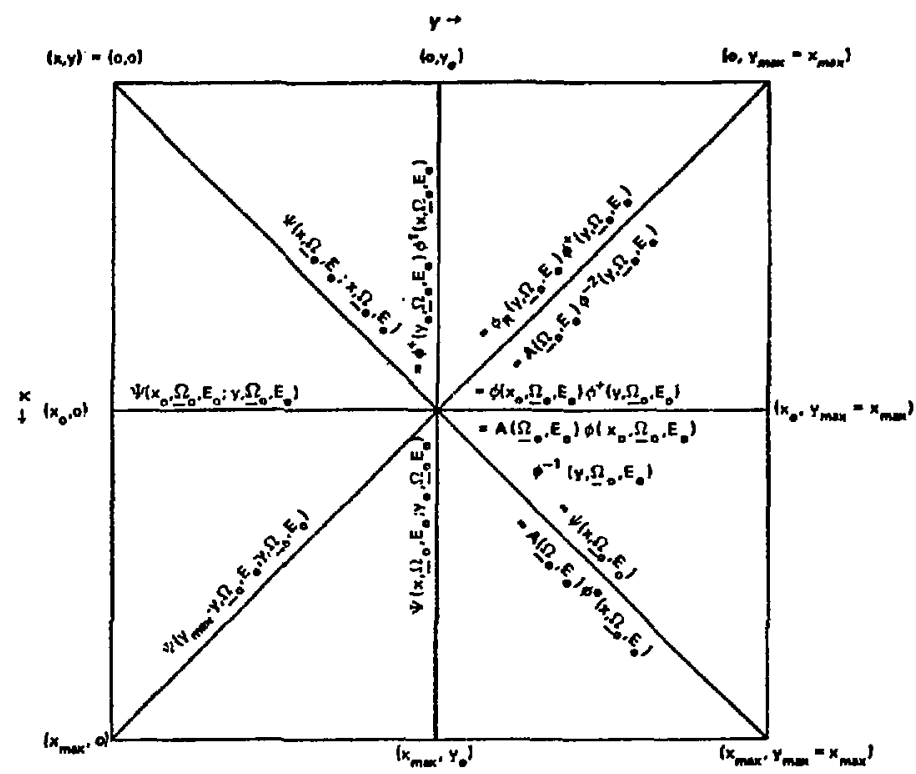

Fig. 16. Information storage for $\phi, \phi^{+}, \psi$, and $\phi_{R^{+}} \phi^{+}$in submatrix of $\Psi$ matrix.

$\psi\left(x_{0}, \Omega_{0}, E_{0} ; y, \Omega_{0}, E_{0}\right)$, and $\psi\left(y_{\max }-y, \Omega_{0}, E_{0} ; y, \Omega_{0}, E_{0}\right)$, where $x$ and $y$ vary between 0 and $x_{\max }$. From the definition in Eq. (253) of the generalized contributon flux and from Eqs. (256)-(258) and (260), it can be seen that

$$
\begin{aligned}
& \psi\left(x, \underline{\Omega}_{0}, E_{0} ; x, \underline{\Omega}_{0}, E_{0}\right)=\phi\left(x, \Omega_{0}, E_{0}\right) \phi^{+}\left(x, \underline{\Omega}_{0}, E_{0}\right) \\
& =\psi\left(x, \underline{\Omega}_{0}, E_{0}\right)=A\left(\Omega_{0}, E_{0}\right) \phi^{0}\left(x, \underline{\Omega}_{0}, E_{0}\right), \\
& \Psi\left(x, \Omega_{0}, E_{0} ; y_{0}, \Omega_{0}, E_{0}\right)=\phi^{1}\left(x, \Omega_{0} E_{0}\right) \phi^{+}\left(y_{0}, \Omega_{0}, E_{0}\right), \\
& \Psi\left(x_{0}, \Omega_{0}, E_{0} ; y, \Omega_{0}, E_{0}\right)=\phi\left(x_{0}, \Omega_{0}, E_{0}\right) \phi^{+}\left(y, \Omega_{0}, E_{0}\right) \\
& =A\left(\underline{\Omega}_{0}, E_{0}\right) \phi\left(x_{0}, \underline{\Omega}_{0}, E_{0}\right) \phi^{-1}\left(y, \underline{\Omega}_{0}, E_{0}\right),
\end{aligned}
$$

ard
Comparison of Eqs. (261)-(264) with Fig. 16 shows the following:

(1) the spatial variation of $\Psi$ is slowest along the main diagonal, where $\Psi \equiv \psi=$ $A\left(\Omega_{0}, E_{0}\right) \phi^{\circ}\left(x, \Omega_{0}, E_{0}\right)=$ constant with respect to $x$;

(2) the spatial variation of $\Psi$ is most rapid along the other diagonal, where $\psi \equiv \phi_{\mathrm{R}} \phi^{+}$ $=A\left(\Omega_{0}, E_{0}\right) \phi^{-2}\left(y, \Omega_{0}, E_{0}\right)$; and

(3) the spatial variation of $\%$ iles midway between that of $\psi$ and $\phi_{R^{+}} \phi^{+}$along the vertical line through $y=y_{0}$, where $\Psi \equiv \phi^{+}\left(y_{0}, \Omega_{0}, E_{0}\right) \phi^{1}\left(x, \Omega_{0}, E_{0}\right)$, and along the horizontal line through $x=x_{0}$, where $\Psi=A\left(\Omega_{0}, E_{0}\right) \phi\left(x_{0}, \Omega_{0}, E_{0}\right) \phi^{-1}\left(y, \Omega_{0}, E_{0}\right)$.

The conclusion which may be drawn from this is that a function $X$, in order to vary more slowly than $\phi$ and $\phi^{+}$, should be represented by data that lies on

$$
\begin{aligned}
& \Psi\left(y_{\max }-y, \underline{\Omega}_{0}, E_{0} ; y, \underline{\Omega}_{0}, E_{0}\right)=\phi\left(y_{\max }-y, \Omega_{0}, E_{0}\right) \phi^{+}\left(y, \underline{\Omega}_{0}, E_{0}\right) \\
& \quad=\phi_{R}\left(y, \underline{\Omega}_{0}, E_{0}\right) \phi^{+}\left(y, \underline{\Omega}_{0}, E_{0}\right) \\
& \quad=A\left(\underline{\Omega}_{0}, E_{0}\right) \phi^{-2}\left(y, \underline{\Omega}_{0} E_{0}\right) .
\end{aligned}
$$


some curve which falls entirely within the upper left and lower right quadrants of the submatrix in Fig. 16. Since $X$ and $\psi$ must be independent, this curve cannt coincide with the main diagonal, where $\Psi \equiv \psi$ is spatially a constant. The ideal function $X$ might, therefore, be a perturbed contributon flux, namely,

$$
x=\psi+\delta \psi,
$$

where $|\delta \psi| \ll \psi$. If such a quantity $x$ could be found, then 1 t might be possible to reformulate the forward and adjoint problems in terms of two new functions, and $\chi$, which vary more slowly than $\phi$ and $\phi^{+}$over much of the system. Since $\phi(x)$ is roughly exponential in a scattering medium which is Eree of external sources and detectors (i.e., an $0^{\prime}$ region), this discussion can also serve as a guide for choosing $X$ in the more general problem with scattering. The details of determining a specific function $X$ have not been carried out to date, however.

\section{SUMMARY AND CONCLUSIONS}

There were three main goals in this research on contributon transport. The first was to more fully describe the physics of contributon transport. To accomplish this we have written for the contributon angular flux $\psi$ a Boltzmann-like transport equation, which has an interpretation similar to that of the Boltzmann transport equation for the forward flux $\phi$. In writing this equation for $\psi$, special contributon scattering and absorption cross sections were introduced, and examination of the definftions of these cross sections has revealed some of the peculiar properties of contributon interactions with the medium and vacuum boundaries.

The second and third goals were to Identify contributon transport equations and their boundary conditions and then to Identify posstble approaches to solving these equations deterministically. Four such equations were obtained, but only two were actively Investigated with a view toward deterministic solutions. The solution approaches were designed to exploit the fact that $\psi$ varles more slowly than $\phi$ and $\phi^{+}$over much of the systern.

The first transport "equation" obtained for $\psi$ is actually a set of four differential equations, namely a linear first-order equation, two linear secondorder equations, and a nonlinear third-order equation. The appropriate equation for $\psi$ from this set is determined on a region-by-region basis from the forward and adjoint source distributions, $\mathrm{S}$ and $\mathrm{s}^{+}$, in that region. The first and second-order equations are applicable in pure absorber regions only, and the third-order equation is applicable in scattering regions and certain pure absorber reglons. It has been possible to completely specify all boundary conditions on $\psi$, without having to solve first for $\phi$ and $\phi^{+}$, except for problems containing source and detector-free pure absorbel regions. For such regions the contributon transport problem is degenerate, and a complete set of boundary conditions cannot be specified without solving the forward or adjoint Boltzmann transport equation in the degenerate regions. However, this poses no real difficulty, since the forward and adjoint Boltzmann equations are easily solvable analytically in such regions. The nonlineartty of the third-order equation introduces only a minor complication. The iterative approach that would normally be used because of the nonlinearity is formally equivalent to the source iteration usually employed in solving the forward and adjoint Boltzmann equations in the discrete-ordinates approximation. For this reason, the third-order equation for $\psi$ has been referred to as quasi-iinear. Some problems described entirely by the first and second-order equations have been solved both analytically and numerically with no difficulty, and some simple pure absorber problems described by the thirdorder equation have also been solved analytically. However, attempts to solve the third-order equation numerically by the discrete-ordinates method indicate that the difference equations are susceptible to numerical instability when source discontinulties are present, and this problem is still unresolved. The second transport equation obtalned for $\psi$ is a generalization of the nonlinear equation of Ref. 2 to general phase-space coordinates and to scattering media. A set of boundary conditions was postulated for this equation, but because of the obvlous difficulty involved in solving this equation, it was investigated no further at this time.

The third transport "equation" obtained for $\psi$ consists of a pair of differential equations. These contributon equations were obtained by transforming 
the forward and adjolnt Boltzmann equations in such a way that the resulting equations contain only quantitles which vary slowly in the spatial variable over much of the system. These contributon equations are colved numerically by iterating between the two equations. However, it has been found that unless the contributon difference equations are consistent with the forward and adjoint difference equations, a distortion is introduced into the solution for $\psi$. Consistency can be obtained when the same spatial grid is used for $\psi, \phi$, and $\phi^{+}$, but there is no computational benefit over solving separately for $\phi$ and $\phi^{+}$when a single grid is used. To obtain any benefit from this method, it is necessary to use a multiple grid (acoarse grid for $\psi$ and a finer grid for $\phi$ and $\phi^{+}$). However, when such a multiple grid is employed, an inconsistency is again introduced, the effects of which have not yet been eliminated,

The final transport equation obtained for $\psi$ is an integral equation for a generalized contributon flux. Although no attempt was made to solve this equation, the approach has been useful in describing a method of attacking the general contributon transport problem. This approach indicates that instead of solving for $\phi$ and $\phi^{+}$, one might solve for two other unknown functions, namely the contributon flux $\psi$ and a perturbed contributon flux $\chi$, where $\chi$ must be Independent of $\psi$ in describing the transport problem. Any computational advantage in such an approach would stem from the fact that one would be solving for two functions, $\psi$ and $x$, which vary more slowly that $\phi$ and $\phi^{+}$in the spatial vartables over much of the syster.

Although the goal of solving the contributon transport problem deterministically has not been fully attained, progress has been made in that direction. At this point, two approaches, the quast-1inear thIrd-order approach and the 1terative approach, offer the most potential as deterministic solution techniques. The third-order approach is promising If the stabllity problem, which is probably related to the singularities at source discontinuities, can be resolved. The multiple-grid form of the iterative approach is promising if the inconsistencies can be eliminated or minfmized.

\section{REFERENCES}

1. G. I. Bell and S. Glasstone, Nuclear Reactor Theory, Van Nostrand Reinhold Conpany, New York (1970).

2. S. A. W. Gerst1, "A New Concept for Deep-Penetration Transport Calculations and Two New Forms of the Neutron Transport Equation," Los Alawos Scientific Laboratory report LA-6628-MS (Decenber 1976).

3. M. L. W1llians and W. W. Engle, Jr., "The Concept of Spatial Channel Theory Applied to Reactor Shielding Analysis," Nuclear Sclence and Engineering 62, 92-104 (1977).

4. A. Dub1, S. A. W. Gerst1, and D. J. Dudzlak, "Monte Carlo Aspects of Contributons," Nuclear Sclence and Engineering 68, 19-30 (1978).

5. P. Barbuccl and F. DiPasquantonio, "Exponential Supplementary Equations for $S_{\mathrm{T}}$ Methods: The OneDimensional Case," Nuclear Scîence and Engineering 63, 179-187 (1977).

6. J. Spanier and E. M. Gelbard, Monte Carlo Principles and Neutron Transport Problems, AddisonWesley Publish1ng Company, Reading, Mass. (1969).

7. A. Dubi, Los Alamos Scientific Laboratory, private comunication (1977).

8. T. R. Hill, "ONETRAN, A Discrete Ordinates Finite Element Code for the Solution of the One-Dimensional Multigroup Transport Equation," Los Alamos Scientific Laboratory report LA-5990-MS (June 1975).

9. K. M. Case and P. F. Zweife1, Linear Transport Theory, Addison-Wesley Publishing Company, Reading, Mass. (1967).

10. S. A. W. Gerst1 and J. W. Painter, "Transport Equations for Contributons," Nuclear Science and Engineering 69, 129-131 (1979).

11. A. Gandini, Lab. Teorla e Calcolo Reattori, private communication (1977).

12. S. D. Conte and C. de Boor, Elementary Nui e-ical Analysis, McGraw-Hill Book Company, New York (1972).

13. E. W. Larsen, Los Alamos Scientific Laboratory, private communication (1978).

14. J. Matthews and R. L. Walker, Mathematical Methods of Physics, W. A. Benjamin, Inc., New York (1970). 
The numerical method, described in Section IV.A.7, Sur solving the third-order quasi-linear contribiston transport equation by first solving the associated second-order differential equation for $f(x, \mu)$, is susceptible to numerical instability in the form of extreme sensitivity of the solutior $f(x, \mu)$ to the choice of the spatial cell width $\Delta x$. It is postulated that this instability is due to the existence of critical values of $\Delta x$ for which the determinant of the coefficient matrix of the system of difference equation vanishes. In this appendix, it will be demonstrated by a simple example that such critical values $\Delta x_{c}$ can, in fact, exist.

Consider a pure absorber problem in slab geometry with a uniform absorption cross section $\sigma_{A}$ and with the source and detector distributions,

$$
Q(x, \mu)= \begin{cases}Q_{1}>0,0<x<t \\ Q_{2}>0, t<x<2 t\end{cases}
$$

and

$$
R(x, \mu)= \begin{cases}R_{1}>0, & 0<x<t \\ R_{2}>0, & t<x<2 t,\end{cases}
$$

where $Q_{1} R_{2} \neq Q_{2} R_{1}$. To simplify the analysis, the fewest possible spatial mesh points (four) will be used. Two of these mesh points, $x_{1}=0$ and $x_{4}=2 t$, are located at the external boundaries, and the other two mesh points, $x_{2}=t^{-}$and $x_{3}=t^{+}$, are 10cated at the internal boundary, $x=t$. If the external boundaries are vacuum boundaries, ther Eqs. (147) and (149) for $\mu>0$ reduce to

$$
\begin{gathered}
\frac{\mu}{Q_{1} R_{1} \Delta x}\left[\left(\frac{\sigma_{A} \Delta x}{2 \mu}\right)^{2}+\frac{\sigma_{A} \Delta x}{2 \mu}+\frac{1}{2}\right] f_{1}(\mu) \\
-\frac{\mu}{2 Q_{1} R_{1} \Delta x} f_{2}(\mu)=1
\end{gathered}
$$

and

$$
\begin{aligned}
& -\frac{\mu}{2 Q_{2} R_{2} \Delta x} f_{3}(\mu)+\frac{\mu}{Q_{2} R_{2} \Delta x}\left[\left(\frac{\sigma_{A} \Delta x}{2 \mu}\right)^{2}+\frac{\sigma_{A} \Delta x}{2 \mu}+\frac{1}{2}\right] \\
& \cdot f_{4}(\mu)=-1,
\end{aligned}
$$

where $\Delta x=t$. Eqs. (154) and (155) for the interna1 boundary, $x=t$, become

$$
\begin{gathered}
-\frac{\mu}{2 Q_{1} R_{1} \Delta x} f_{1}(\mu)+\frac{\mu}{Q_{1} R_{1} \Delta_{x}}\left[\left(\frac{\sigma_{A} \Delta x}{2 \mu}\right)^{2}\right. \\
\left.+\frac{\sigma_{A} \Delta x}{2 \mu}\left(\frac{Q_{1} R_{2}+Q_{2} R_{1}}{Q_{2} R_{1}-Q_{1} R_{2}}\right)+\frac{1}{2}\right] f_{2}(\mu) \\
+\frac{\sigma_{A}}{Q_{2} R_{1}-Q_{1} R_{2}} f_{3}(\mu)=-1
\end{gathered}
$$

and

$$
\begin{gathered}
\frac{\sigma_{A}}{Q_{2} R_{1}-Q_{1} R_{2}} f_{2}(\mu)+\frac{\mu}{Q_{2} R_{2} \Delta x}\left[\left(\frac{\sigma_{A} \Delta x}{2 \mu}\right)^{2}\right. \\
\left.+\frac{\sigma_{A} \Delta x}{\angle \mu}\left(\frac{Q_{1} R_{2}+Q_{2} R_{1}}{Q_{2} R_{1}-Q_{1} R_{2}}\right)+\frac{1}{2}\right] f_{3}(\mu) \\
-\frac{\mu}{2 Q_{2} R_{2} \Delta x} F_{4}(\mu)=1 .
\end{gathered}
$$

We now define the following quantities:

$$
\begin{aligned}
& \beta=Q_{2} R_{1}-Q_{1} R_{2} \neq 0, \\
& \delta_{1}=\frac{\mu}{Q_{1} R_{1} \Delta x}, \\
& \delta_{2}=\frac{\mu}{Q_{2} R_{2} \Delta x}, \\
& n=\frac{\sigma_{A} \Delta x}{2 \mu},
\end{aligned}
$$

and

$$
Y=\left(\frac{\sigma_{A} \Delta x}{2 \mu}\right)^{2}+\frac{\sigma_{A} \Delta x}{2 u}+\frac{1}{2}
$$

$$
=n^{2}+n+\frac{1}{2} \text {. }
$$


With these definitions the system of difference equations $(A 3)-(A 6)$ can be written as

$\left[\begin{array}{cccc}\delta_{1} \gamma & -\frac{1}{2} \delta_{1} & 0 & 0 \\ -\frac{1}{2} \delta_{1} & \delta_{1}\left(\gamma+2 \eta \frac{Q_{1} R_{2}}{B}\right) & \frac{\sigma_{A}}{B} & 0 \\ 0 & \frac{\sigma_{A}}{B} & \delta_{2}\left(\gamma+2 n \frac{Q_{1} R_{2}}{B}\right) & -\frac{1}{2} \delta_{2} \\ 0 & 0 & -\frac{1}{2} \delta_{2} & \delta_{2} \gamma\end{array}\right]\left[\begin{array}{c}f_{1} \\ f_{2} \\ f_{3} \\ f_{4}\end{array}\right]=\left[\begin{array}{c}1 \\ -1 \\ 1 \\ -1\end{array}\right]$

The determinant of the coefflclent mitrix in Eq. (A12) is easily found to be

$$
\begin{aligned}
\Delta= & \left(\delta_{1} \delta_{2}\right)^{2}\left[\gamma^{2}+\left(2 r_{1} \frac{Q_{I} R_{2}}{B}+\frac{\sigma_{A}}{\beta \sqrt{\delta_{1} \delta_{2}}}\right) \gamma-\frac{1}{4}\right] \\
& \cdot\left[\gamma^{2}+\left(2 n \frac{Q_{1} R_{2}}{B}-\frac{\sigma_{A}}{\beta \sqrt{\delta_{1} \delta_{2}}}\right) \gamma-\frac{1}{4}\right] .
\end{aligned}
$$

If $\Delta$ is to vanish, then one of the two conditions,

$$
\gamma^{2}+\left(2 n \frac{Q_{1} R_{2}}{\beta}+\frac{\sigma_{A}}{\beta \sqrt{\delta_{1} \delta_{2}}}\right) \gamma-\frac{1}{4}=0
$$

or

$$
\gamma^{2}+\left(2 n \frac{Q_{1} R_{2}}{\beta}-\frac{\sigma_{A}}{B \sqrt{\delta_{1} \delta_{2}}}\right) \gamma-\frac{1}{4}=0 \text {, }
$$

must be satisfied since $\delta_{1}$ and $\delta_{2}$ are both non-zero for $\mu \neq 0$. If Eqs. (AB)-(A10) are used in Eqs. (A14) and (A15), then the two conditions become

$$
\gamma^{2}+\frac{2 \pi}{B}\left(Q_{1} R_{2}+\sqrt{Q_{1} Q_{2} R_{1} R_{2}}\right) \gamma-\frac{1}{4}=0
$$

and

$$
\gamma^{2}+\frac{2 n}{\beta}\left(Q_{1} R_{2}-\sqrt{Q_{1} Q_{2} R_{1} R_{2}}\right) \gamma-\frac{1}{4}=0 .
$$

If Eqs. (A7) and (A11) are then used in Eqs. (A16) and (A17), we obtain for the two conditions

$$
\eta\left(n^{3}+\xi_{+} n^{2}+\xi_{+} n+\frac{1}{2} \xi_{+}\right)=0
$$

and

$$
n\left(n^{3}+\xi_{-} n^{2}+\xi_{-} n+\frac{1}{2} \xi_{-}\right)=0 \text {, }
$$

where

$$
\xi_{ \pm}=2 \frac{Q_{2}^{R_{1}}}{\beta}\left(1 \pm \sqrt{\frac{Q_{1}^{R_{2}}}{Q_{2} R_{1}}}\right) .
$$

Since we wish to demonstrate the existence of critical values $\Delta x_{c}>0$, for which $\Delta$ of $E q$. (A13) vanishes, it suffices to show for $\mu>0$ that at least one of the two equations, (A18) or (A19), has a solution $\eta_{c}>0$. Thus, we ignore the solution, $\eta=0$, and consider only the remaining solutions to $\mathrm{Eq} .(\mathrm{Al} 8$ ) and (Al9), that is, the solutions to

$$
F_{+}(\eta) \equiv \eta^{3}+\xi_{+} n^{2}+\xi_{+} \eta+\frac{1}{2} \xi_{+}=0
$$

and

$$
F_{-}(n) \equiv n^{3}+\xi_{-} n^{2}+\xi_{-} n+\frac{1}{2} \xi_{-}=0 .
$$

It is first noted that, for large $\eta>0$, both $F_{+}(\eta)$ and $F_{-}(n)$ are asymptotic to $\eta^{3}$; that is,

$$
F_{ \pm}(n) \sim n^{3}>0 \text { for } n \gg 0
$$

Thus, there must be at least one $\eta_{c}>0$ if either $F_{ \pm}(0)<0$ or $F_{-}(0)<0$. From Eqs. (A2I) and (A22),

$$
F_{+}(0)=\frac{1}{2} \xi_{+}=\frac{\sqrt{Q_{2} R_{1}}}{\sqrt{Q_{2} R_{1}}-\sqrt{Q_{1} R_{2}}}
$$

and

$$
F_{-}(0)=\frac{1}{2} \xi_{-}=\frac{\sqrt{Q_{2} R_{1}}}{\sqrt{Q_{2} R_{1}}+\sqrt{Q_{1} R_{2}}} .
$$

From Eqs. (A24) and (A25), F_(0) is always greater than zero, but if $Q_{1} R_{2}>Q_{2} R_{1}$, then $F_{+}(0)<0$ and $F_{+}(\eta)$ has at least one zero, 1.e., $F_{+}\left(\eta_{c}\right)=0$ for $\eta_{c}>0$. Consequently, for $\mu>0$ and $Q_{1} R_{2}>Q_{2} R_{1}$, there is at least one critical cell size, $\Delta x_{c}>0$, for which the determinant $\Delta$ vanishes. It is possible to continue this analysis and determine the precise number of critical values $\Delta x_{c}$, but this is not necessary to the demonstration of existence. 
If the flux $\phi$ in the forward Boltzmann transport equation is replaced by $f \Psi$, then as shown in Section IV.C.1, the result is the transformed forward equation,

$$
\Omega \cdot \nabla \Psi+\left(\sigma+\frac{1}{f} \Omega \cdot \nabla f\right) \Psi=\frac{S}{f},
$$

where no restrictions are imposed on either $f$ or $\Psi$ at this stage. If the goal is to solve Eq. (BI) for a function $\psi$ which is slowly varying in the spatial variable $\underline{r}$, then the Ideal choice for $f$ is the one which would make $\Psi$ satisfy the equation,

$$
\underline{\Omega} \cdot \nabla \Psi=0
$$

When Eq. (B2) is inserted into Eq. (31), we see that the ideal $f$ should satisfy

$$
\underline{\Omega} \cdot \nabla f+\sigma f=S \text {, }
$$

which is just the forward Boltzmann equation with $\phi$ replaced by $f$. This result implies that an ideal choice for $f$ is

$$
\mathbf{f} \equiv \phi
$$

Since per definition

$$
\Psi=\frac{\phi}{\mathbf{f}},
$$

the choice of $f$ according to Eq. (B4) gives

$$
\Psi \equiv 1 \text {. }
$$

In an iterative solution scheme for the forward Boltzmann transport equation, one generally knows only the latest iterate $\phi^{(k-1)}$ and not the converged flux $\phi$. Consequently, in an iterative solution, we choose $\mathrm{f}^{(\mathrm{k}-1)}$ to be

$$
f^{(k-1)} \equiv \phi^{(k-1)}
$$

and $\psi^{(k)}$ to be

$$
\psi^{(k)} \equiv \frac{\phi^{(k)}}{\phi^{(k-1)}} \text {, }
$$

where $\phi^{(k)}$ satisfies the forward Boltzmann equation,

$$
\Omega \cdot \nabla \phi^{(k)}+\sigma \phi^{(k)}=s^{(k-1)},
$$

in each iteration $k$. Though the use of Eqs. (B7)(B9), Eq. (B1) becomes

$$
\underline{\Omega} \cdot \nabla \psi^{(k)}+\left[\frac{s^{(k-2)}}{\phi^{(k-1)}}\right] \nabla^{(k)}=\frac{s^{(k-1)}}{\phi^{(k-1)}}
$$

for iteration $k$. The vacuum boundary condition for $\psi^{(k)}$ is chosen to be

$$
\psi^{(\mathrm{k})}=\frac{\mathrm{s}^{(\mathrm{k}-1)}}{\mathrm{s}^{(\mathrm{k}-2)}} \quad \text { for } \hat{\mathrm{n}} \cdot \underline{\Omega}<0 \text {, }
$$

since this is the only value of $\psi^{(k)}$ for which $\Omega \cdot \nabla \psi^{(\mathrm{k})}$ can be bounded for incoming directions. This is easily seen when Eq. (B11) is inserted into Eq. (B10). In view of Eqs. (B8) and (B11), ${\psi^{(k)}}^{(\mathrm{k})}$ verges to $\Psi \equiv 1$ throughout phase space when $\phi^{(k)}$ converges to $\phi$.

The approach defined by Eqs. (B7) - (B11) is just one in a class of approaches based upon the possible choices for $f$ in Eq. (BI). Another is the contributon approach as discussed in Section IV.C, In which $f$ is chosen to be $1 / \phi^{+}$. The above exercise provides the following insight: If $f$ is chosen as $\phi$ then $\tilde{\psi}$ in Eq. (BI) is constant throughout phase space. However, if $\psi$ is to be interpreted as the contributon flux, $\psi=\phi \phi^{+}$, the solution $\psi^{\prime}=A=$ const. Implies that $\phi^{+} \equiv A / \phi$. This relationship between forward and adjoint flux holds only in special cases, e.g., in a source and detector-free region of a pure absorber problem ("O-region"). On the other hand, if a problem contains scattering sources, its solution for the contributon flux $\tilde{\psi}$ is most nearly constant in reglons where only very little scattering occurs, 1.e., where $\sigma_{s} / \sigma_{\text {TOT }} \ll 1$. In these cases a solution method for the appropriate contributon transport equation which exploits the slow varlation of $\Psi$ is expected to be benefictal. 
The discrete-ordinates cransport equation for the forward flux $\phi$ in slab geometry is

$$
\mu_{m}\left[\phi_{m, 1+\frac{1}{2}}-\phi_{m, 1-\frac{1}{s}}\right]+\sigma_{1} \phi_{m i} \Delta x_{1}=S_{m 1} \Delta x_{1},
$$

and the consistent supplementary equation for $\phi$ is

$$
\frac{\phi_{m 1}^{2}}{\phi_{m, 1+\frac{1}{2}} \phi_{m, 1-\frac{1}{2}}}=C_{m 1}^{2}
$$

In these equations, $\phi_{\mathrm{mf}}$ is the cell-averaged flux and $\phi_{m, 1 \pm \frac{1}{2}}$ are the cell boundary fluxes. If the flux is assumed to have an exponential shape across the ce11, then the cell center flux $\phi_{\pi 1}^{(c)}$ satisfles $^{5}$

$$
\frac{\left[\phi_{m 1}^{(c)}\right]^{2}}{\phi_{m, 1+x_{2}^{\phi} m, 1-\frac{1}{2}}}=1 .
$$

Thus, taking the ratio of corresponding sides of Egs. (C2) and (C3) shows that

$$
c_{m i}=\frac{\phi_{m 1}}{\phi_{m 1}^{(c)}}=\frac{1}{\phi_{m 1}^{(c)} \Delta x_{i}} \int_{x_{1-\frac{1}{2}}}^{x_{1+\frac{1}{2}}} \phi_{m 1}(x) d x
$$

If the flux $\phi_{m I}(x)$ has an exponential shape across the cell. If the flux shape within cell $i$ is given by

$$
\phi_{m 1}(x)=\phi_{m i}^{(c)} e^{-\sigma_{i}\left(x-x_{1}\right) / v_{m 1}},
$$

It is easily shown by inserting Eq. (CS) Into Eq.

$$
\text { (C4) that } C_{m 1}=\frac{\sinh \frac{\sigma_{1} \Delta x_{1}}{2 v_{m 1}}}{\frac{\sigma_{1} \Delta x_{1}}{2 \nu_{m 1}}} \text {, }
$$

where $\nu_{m !}$ is an, as yet, unspecifled cell paramecer.

Frod equation (C6), it can be seen that there is a basic inconsistency if $\phi_{\mathrm{mi}}(x)$ is assumed to have an exponential shape and if $c_{m i} \equiv 1$, unless $\phi_{m 1}=$ $\phi_{m 1}^{(c)}$ or $\frac{\sigma_{1} \Delta x_{1}}{2 \nu_{m 1}}=0$. Indeed, the accuracy of the solution can be very sensitive to the value chosen for $C_{m i}$, especially for deep-penetxation problems with large $\sigma_{1} \Delta x_{1}$. To dewonstrate this sensitivity, the results for a simple monoenergetic deep-penetration problem (50 mean free paths) are presented. The geometry and parameters for this problem are shown in Figure 8 and the results are presented in Figure 17 for the $\mathrm{S}_{4}$ approximation. The quantity plotted is the ratio of the integral response $I$, computed from Eq. (5c), for a constant mesh size $\sigma \Delta x .=1.0$ in the 0 ' region to the "exact" incegral response obtained with $G \Delta x=0.05$ in the $O^{\prime}$ region. The scactering-to-total ratio is varied from zero to 0.9. The vartous curves in Figure 17 correspond to the different values used for $c_{m i}$, namely:

(1) $c_{m i}=1$, (2) $c_{m i}=\frac{\sinh \frac{\sigma_{i} \Delta x_{i}}{2 \mu_{m}}}{\frac{\sigma_{i} \Delta x_{i}}{2 \mu_{m}}} \quad \begin{aligned} & \text { a source-free pure absorber } \\ & \text { region, in which the exact }\end{aligned}$ flux solution in the $0^{\prime}-$ region is given by $\mathrm{Eq}$. (C5) with $v_{m i}=\mu_{m}$,

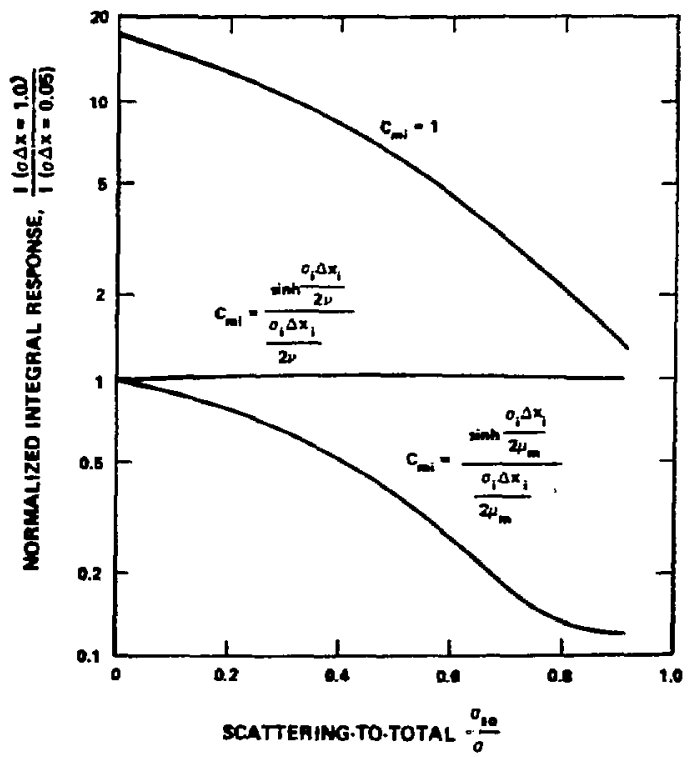

Fig. 17. Sensitivity of Integral Response to the choice of $\mathrm{C}_{\mathrm{ml}}$. 
and

(3) $C_{m i}=\frac{\sinh \frac{\sigma_{i} \Delta x_{i}}{2 v}}{\frac{\sigma_{i} \Delta x_{i}}{2 v}}$,

where $v$ is the discrete Case eigenvalue ${ }^{9}$ appropiate for the quadrature set being used; that is, $v$ is the solution to

$$
2 \frac{\sigma_{s 0}}{\sigma} \sum_{m=1}^{M / 2} \frac{w_{m}}{1-\left(\frac{N_{m}}{v}\right)^{2}}=1,
$$

where $\max _{\mathrm{m}}\left|\mu_{\mathrm{m}}\right|<v<\infty$.

Figure 17 shows the extreme sensitivity of the accuracy of the solution to the choice of $\mathrm{C}_{\mathrm{mi}}$. As might be expected, $C_{m i}=1$ is more appropriate for problems with little absorption (close to a pure scatterer), and it is not surprising that $I$ is in- creasingly overestimated as more absorption is introduced into the problem. The second choice for $c_{m i}$, with $v_{m i}=\mu_{m}$, is more appropriate for pure absorber problems, so it is not surprising that I is increasingly underestinated as more scattering is introduced into the problem. Since setting $y$ eqqual to the discrete Case eigenvalue closely approximates the exact solution, it is nnt surprising that the best resuits for I are obtained with the chird choice for $C_{m i}$.

These results do nat show how to choose $\nu_{m i}$ For regions that contain external sources, since this Case eigenvalue method does not apply in such regions. The results do show, however, the need to study methods for choosing $\mathrm{C}_{m i}$, since the accuracy of the solution is extramely sensitive to the choice of $\mathrm{C}_{\mathrm{mi}}$. 